NATIONAL LABORATORY

\title{
Medium Truck Duty Cycle Data from Real-World Driving Environments: Project Interim Report
}

\section{January 2011}

\section{Prepared by}

Oscar Franzese, Mary Beth Lascurain, Gary Capps

R\&D Staff

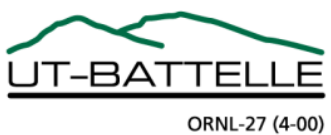




\section{DOCUMENT AVAILABILITY}

Reports produced after January 1, 1996, are generally available free via the U.S. Department of Energy (DOE) Information Bridge.

Web site http://www.osti.gov/bridge

Reports produced before January 1, 1996, may be purchased by members of the public from the following source.

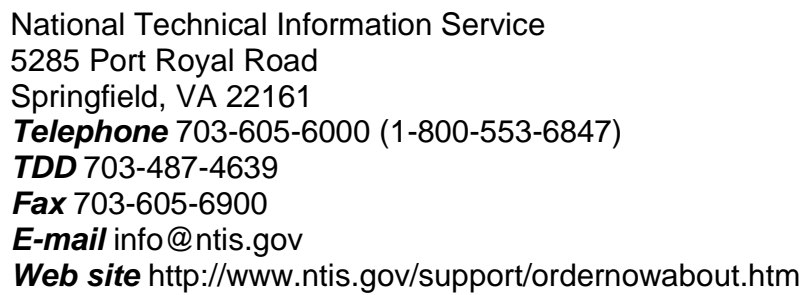

Reports are available to DOE employees, DOE contractors, Energy Technology Data Exchange (ETDE) representatives, and International Nuclear Information System (INIS) representatives from the following source.

Office of Scientific and Technical Information

P.O. Box 62

Oak Ridge, TN 37831

Telephone 865-576-8401

Fax 865-576-5728

E-mail reports@osti.gov

Web site http://www.osti.gov/contact.html

This report was prepared as an account of work sponsored by an agency of the United States Government. Neither the United States Government nor any agency thereof, nor any of their employees, makes any warranty, express or implied, or assumes any legal liability or responsibility for the accuracy, completeness, or usefulness of any information, apparatus, product, or process disclosed, or represents that its use would not infringe privately owned rights. Reference herein to any specific commercial product, process, or service by trade name, trademark, manufacturer, or otherwise, does not necessarily constitute or imply its endorsement, recommendation, or favoring by the United States Government or any agency thereof. The views and opinions of authors expressed herein do not necessarily state or reflect those of the United States Government or any agency thereof. 
Vehicle Systems Program

\title{
MEDIUM TRUCK DUTY CYCLE DATA FROM REAL-WORLD DRIVING ENVIRONMENTS: PROJECT INTERIM REPORT
}

\author{
Oscar Franzese \\ Mary Beth Lascurain \\ Gary Capps
}

Date Published: January 2011

\author{
Prepared by \\ OAK RIDGE NATIONAL LABORATORY \\ Oak Ridge, Tennessee 37831-6283 \\ managed by \\ UT-BATTELLE, LLC \\ for the \\ U.S. DEPARTMENT OF ENERGY \\ under contract DE-AC05-00OR22725
}





\section{CONTENTS}

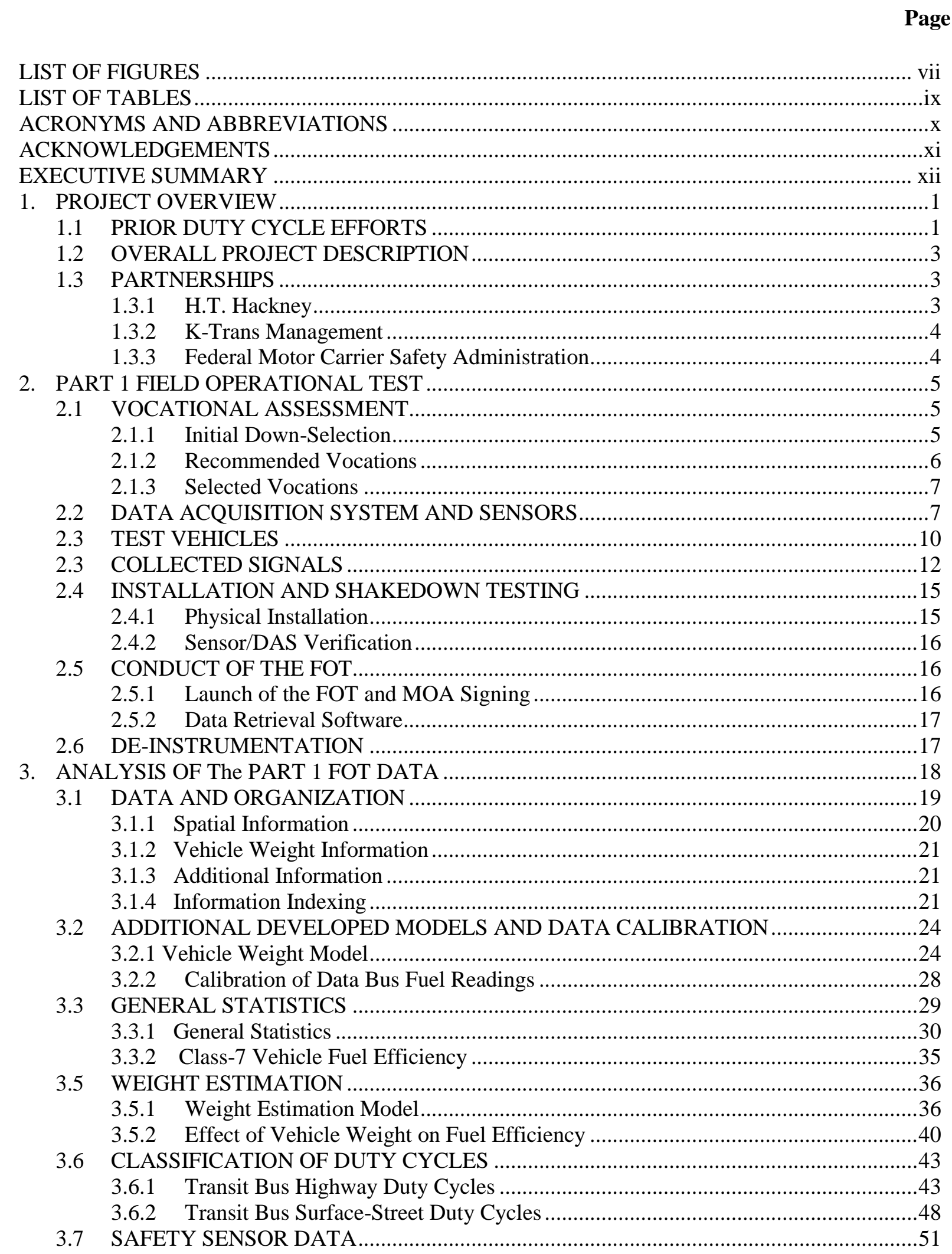




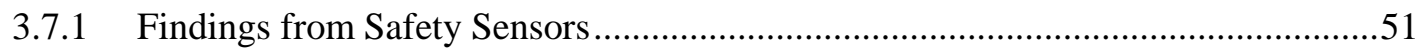

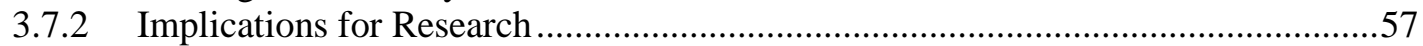

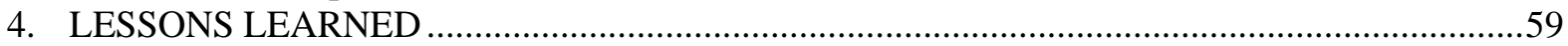

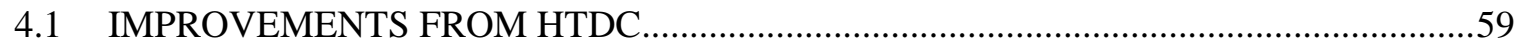

4.1.1 Modifications to Data Acquisition System..........................................................59

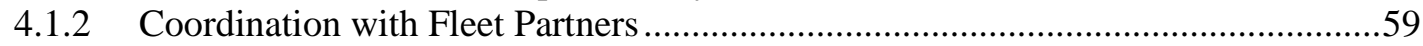

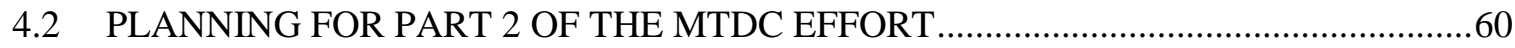

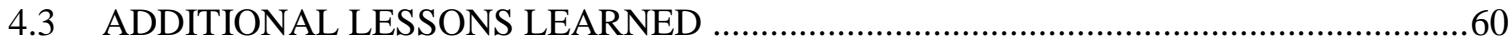

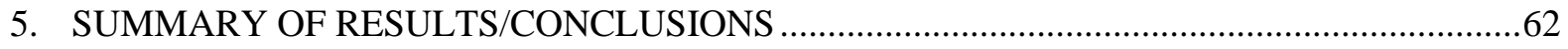

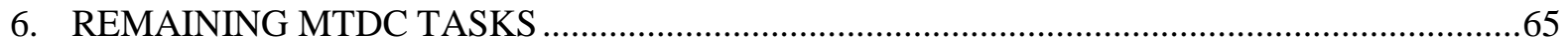

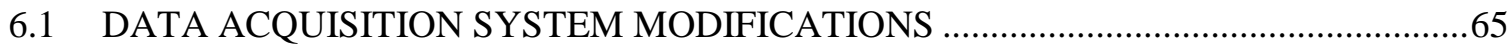

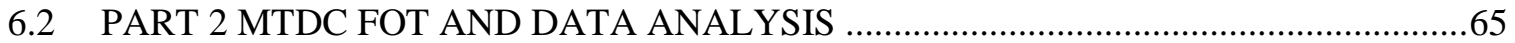

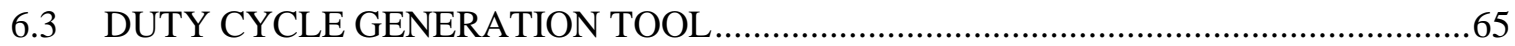

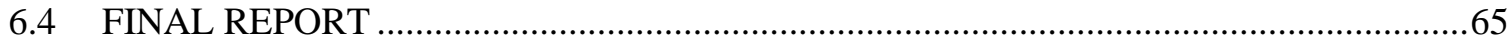




\section{LIST OF FIGURES}

\section{Page}

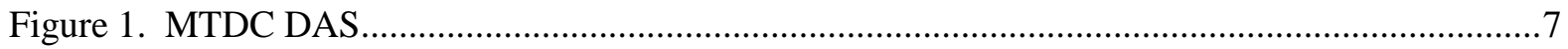

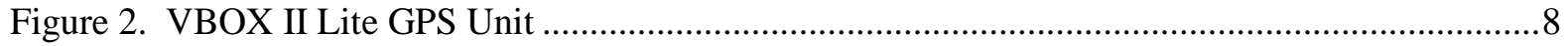

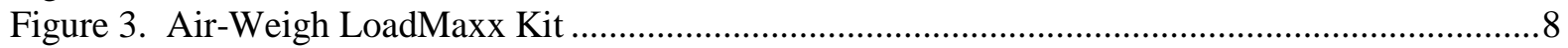

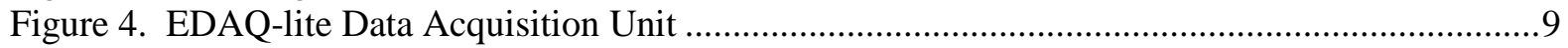

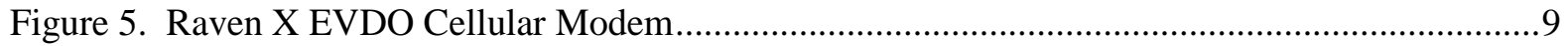

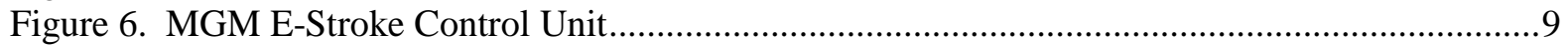

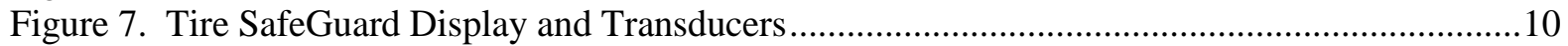

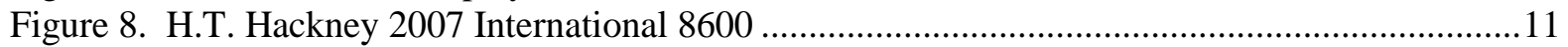

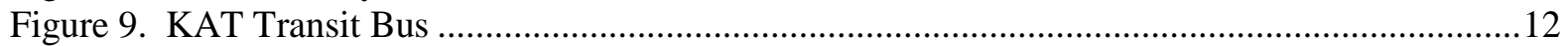

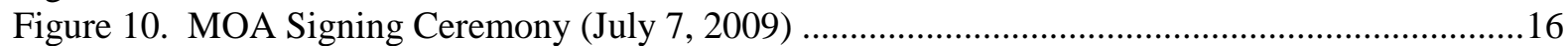

Figure 11. Routes of Participating H.T. Hackney Trucks (East Tennessee Area) ..............................18

Figure 12. Routes of Participating KAT Buses (Knoxville, Tennessee Area) ...................................18

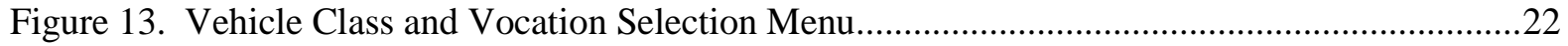

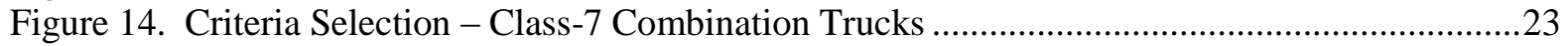

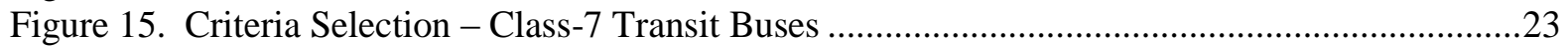

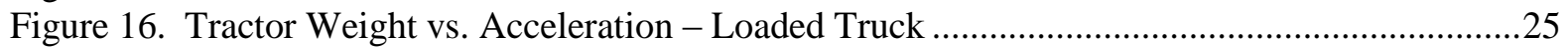

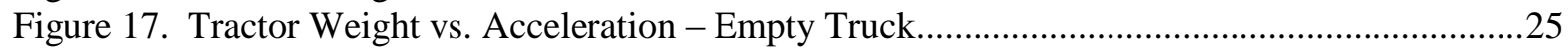

Figure 18. Linear Model for Tractor Weight vs. Acceleration - Loaded Truck ................................26

Figure 19. Linear Model for Tractor Weight vs. Acceleration - Empty Truck ...................................26

Figure 20. Gallons of Fuel Used - Manual Log Values vs. EOBR-reported Values...........................29

Figure 21. Gallons of Fuel Used - EOBR-Reported Values vs. DAS-recorded Values......................29

Figure 22. Combination Truck 1 (Vehicle 1) Coverage Area...............................................................30

Figure 23. Combination Truck 2 (Vehicle 2) Coverage Area...........................................................

Figure 24. Combination Truck 3 (Vehicle 3) Coverage Area............................................................31

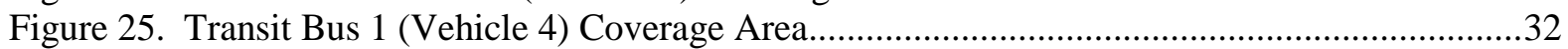

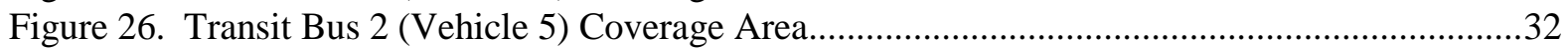

Figure 27. Transit Bus 3 (Vehicle 6) Coverage Area......................................................................33

Figure 28. Combination Truck 1 (Vehicle 1) Frequency Distributions of Measured Steer/Drive

Axle Weights and Modeled Gross Vehicle Weight ......................................................................

Figure 29. Combination Truck 2 (Vehicle 2) Frequency Distributions of Measured Steer/Drive

Axle Weights and Modeled Gross Vehicle Weight .................................................................. 37

Figure 30. Combination Truck 3 (Vehicle 3) Frequency Distributions of Measured Steer/Drive

Axle Weights and Modeled Gross Vehicle Weight ................................................................. 37

Figure 31. Transit Bus 1 (Vehicle 4) Frequency Distribution of Gross Vehicle Weight......................38

Figure 32. Transit Bus 2 (Vehicle 5) Frequency Distribution of Gross Vehicle Weight......................39

Figure 33. Transit Bus 3 (Vehicle 6) Frequency Distribution of Gross Vehicle Weight.....................39

Figure 34. Fuel Efficiency Frequency Distributions as a Function of Load Level - Combination

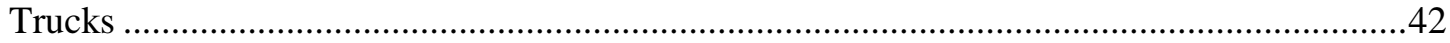

Figure 35. Fuel Efficiency Frequency Distributions as a Function of Load Level - Transit Buses ....43

Figure 36. Transit Bus Highway Duty Cycle Route ........................................................................44

Figure 37. Transit Bus Highway Duty Cycle (DC) Time-of-Day Frequency Distribution .................44

Figure 38. Transit Bus Highway Duty Cycle (DC) Duration Frequency Distribution .......................45

Figure 39. Transit Bus Highway Duty Cycle (DC) Minimum Speed Frequency Distribution............46

Figure 40. Top Five Maximum Average Speed Transit Bus Highway Duty Cycles ..........................47

Figure 41. Bottom Five Maximum Average Speed Transit Bus Highway Duty Cycles ....................47

Figure 42. Transit Bus Surface Street Duty Cycle Route .............................................................48

Figure 43. Transit Bus Surface Street Duty Cycle (DC) Duration Frequency Distribution ................49 
Figure 44. Top Five Maximum Average Speed Transit Bus Surface Street Duty Cycles ...................50

Figure 45. Bottom Five Maximum Average Speed Transit Bus Surface Street Duty Cycles .............50

Figure 46. Distribution of Brake Stroke Status Codes ........................................................................51

Figure 47. Proportion of Braking Events in MTDC Combination Vehicle Duty Cycles ...................52

Figure 48. Distribution of Brake Application Pressure for MTDC Combination Vehicles.................52

Figure 50. Sample Time History of Speed and Brake Application Pressure …..................................53

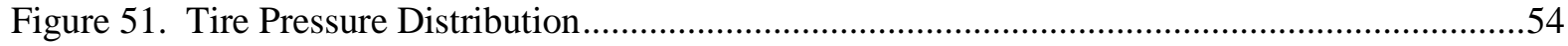

Figure 52. Distribution of Tire Pressure Threshold Detection Codes.............................................55

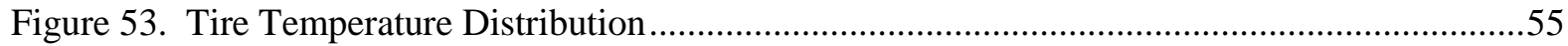

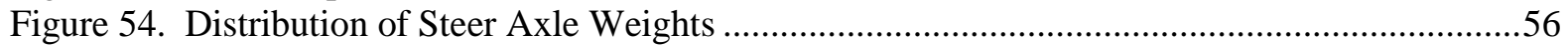

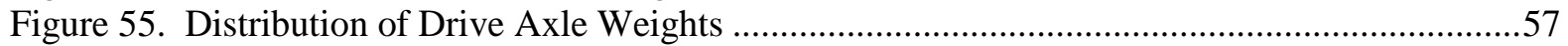

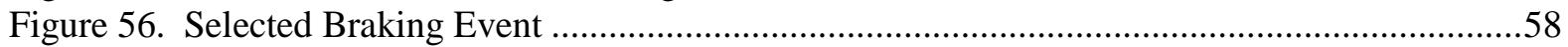




\section{LIST OF TABLES}

\section{Page}

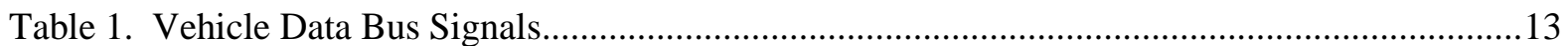

Table 2. Signals from Other Sensors (All Part 1 Vehicles) …............................................................ 14

Table 3. Additional Safety Signals (Tractor of Delivery Vehicles Only) ..........................................15

Table 4. Size and Number of Data Files Generated in the Project ...................................................19

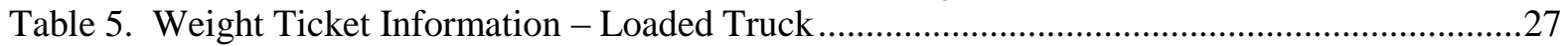

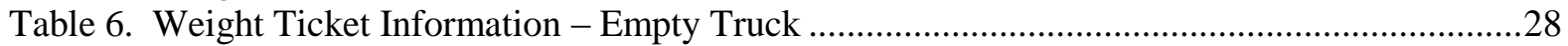

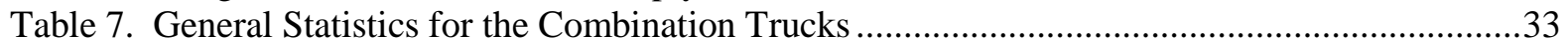

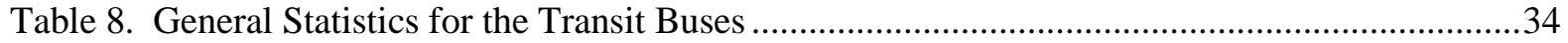

Table 9. Distributions of Time Spent and Fuel Consumed while Idling (Combination Trucks) .........34

Table 10. Distributions of Time Spent and Fuel Consumed while Idling (Transit Buses) .................35

Table 11. Overall and Moving Fuel Efficiency - Combination Trucks ..............................................35

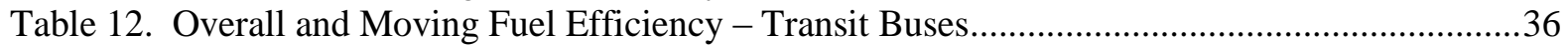

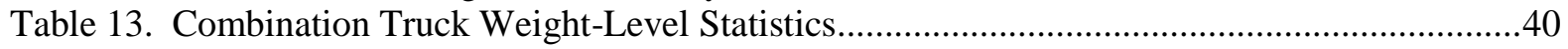

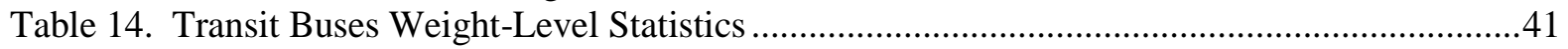

Table 15. Fuel Efficiency as a Function of Load Level - Combination Trucks ...............................41

Table 16. Fuel Efficiency as a Function of Load Level - Transit Buses ..........................................42

Table 17. Transit Bus Highway Duty Cycle Distribution Statistics ................................................45

Table 18. Transit Bus Surface Street Duty Cycle Distribution Statistics.............................................48

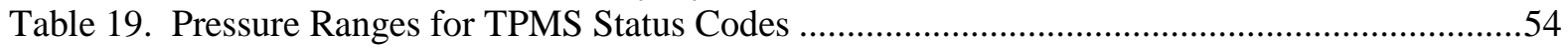




\section{ACRONYMS AND ABBREVIATIONS}

\begin{tabular}{|c|c|}
\hline Term & Definition \\
\hline ASCII & American Standard Code for Information Interchange \\
\hline CTA & Center for Transportation Analysis \\
\hline Dana & Dana Corp. \\
\hline DAS & Data Acquisition System \\
\hline DASIB & Data Acquisition System Interface Box \\
\hline DOE & US Department of Energy \\
\hline DOT & US Department of Transportation \\
\hline DCGenT & Duty Cycle Generation Tool \\
\hline EOBR & Electronic On-Board Recorder \\
\hline EPA & Environmental Protection Agency \\
\hline $\mathrm{FE}$ & Fuel Efficiency \\
\hline FOT & Field Operational Test \\
\hline FMCSA & Federal Motor Carrier Safety Administration \\
\hline GPS & Global Positioning System \\
\hline HTDC & Heavy Truck Duty Cycle \\
\hline KAT & Knoxville Area Transit \\
\hline MARC & Michelin Americas Research and Development Corp. \\
\hline MOA & Memorandum of Agreement \\
\hline MTDC & Medium Truck Duty Cycle \\
\hline NGWBST & New-Generation Wide-Based Single Tires \\
\hline NTRC & National Transportation Research Center \\
\hline ORNL & Oak Ridge National Laboratory \\
\hline PTO & Power Takeoff \\
\hline SDMS & Safety Data Message Set \\
\hline SIE & File Extension Descriptor Assigned by HBM/SoMat \\
\hline SIF & SoMat Information File \\
\hline TPMS & Tire Pressure Monitoring System \\
\hline Vehicle & Test Vehicle Including Tractor and/or Trailer Unit(s) \\
\hline VIUS & Vehicle Inventory and Use Survey \\
\hline WRI & Wireless Roadside Inspection \\
\hline 21CTP & $21^{\text {st }}$ Century Truck Partnership \\
\hline
\end{tabular}




\section{ACKNOWLEDGEMENTS}

The Oak Ridge National Laboratory would like to thank the H.T. Hackney Company and K-Trans Management for making available their trucks to serve as test vehicles for this project. They provided the necessary access to vehicles for the smooth conduct of the Field Operational Test in addition to assisting in equipment installation, troubleshooting, and de-instrumentation. Without the support of H.T.

Hackney's and K-Trans Management's owners, drivers, and technicians, this project would not have been possible.

We would also like to thank Mr. Brian McDivitt (Pensacola Christian College) for his contributions to the project in its early stages through an internship with the US Department of Energy (DOE) Office of Science and the Oak Ridge Institute for Science and Education.

We would also like to thank the Federal Motor Carrier Safety Administration for making possible the collection of additional safety-related signals through their funding for the associated sensors; MGM Brakes for the installation cost of the e-Stroke units; and the Tennessee Highway Patrol of the Tennessee Department of Safety for providing manpower to assist in the calibration of the on-board self-weighing units.

Finally, the project team would like to thank Mr. Lee Slezak (DOE Office of Vehicle Technologies) for his continued support of this important project. Mr. Slezak's encouragement, guidance and enthusiasm, for this work; along with the financial support for its conduct were the foundation which allowed this project to be conducted. We are indebted to Mr. Slezak for his support. 


\section{EXECUTIVE SUMMARY}

Since the early part of the 20th century, the US trucking industry has provided a safe and economical means of moving commodities across the country. At the present time, nearly $80 \%$ of the US domestic freight movement involves the use of trucks. The US Department of Energy (DOE) is spearheading a number of research efforts to improve heavy vehicle fuel efficiencies. This includes research in engine technologies (including hybrid and fuel cell technologies), lightweight materials, advanced fuels, and parasitic loss reductions. In addition, DOE is developing advanced tools and models to support heavy vehicle truck research, and is leading the 21 st Century Truck Partnership whose stretch goals involve a reduction by $50 \%$ of the fuel consumption of heavy vehicles on a ton-mile basis.

This Medium Truck Duty Cycle (MTDC) Project is a critical element in DOE's vision for improved heavy vehicle energy efficiency and is unique in that there is no other existing national database of characteristic duty cycles for medium trucks. It involves the collection of real-world data on medium trucks for various situational characteristics (rural/urban, freeway/arterial, congested/free-flowing, good/bad weather, etc.) and looks at the unique nature of medium trucks' drive cycles (stop-and-go delivery, power takeoff, idle time, short-radius trips), to provide a rich source of data that can contribute to the development of new tools for fuel efficiency and modeling, provide DOE a sound basis upon which to make technology investment decisions, and provide a national archive of real-world-based mediumtruck operational data to support heavy vehicle energy efficiency research. The MTDC project involves a two-part field operational test (FOT). For the Part-1 FOT, three vehicles, each from two vocations (urban transit and dry-box delivery) were instrumented for one year of data collection. The Part-2 FOT will involve the towing/recovery and utility vocations.

The vehicles participating in the MTDC project are doing so through gratis partnerships in return for early access to the results of this study. Partnerships such as these are critical to FOTs in which real-world data is being collected. In Part 1 of the project, Oak Ridge National Laboratory (ORNL) established partnerships with the H.T. Hackney Company, one of the largest wholesale distributors in the country, distributing products to 21 states; and with the Knoxville Area Transit (KAT), the City of Knoxville's transit system, operating services across the city of Knoxville and parts of Knox County. These partnerships and agreements provided ORNL access to three Class-7 2005/2007 International day-cab tractors, model 8600, which regularly haul 28-ft pup trailers (H.T. Hackney Co) and three Class-7 2005 Optima LF-34 buses (KAT), for collection of duty cycle data. In addition, ORNL has collaborated with the Federal Motor Carrier Safety Administration (FMCSA) to determine if there were possible synergies between this duty cycle data collection effort and FMCSA's need to learn more about the operation and duty cycles of the second-largest fuel consuming commercial vehicle category in the US. FMCSA's primary interest was in collecting safety data relative to the driver, carrier, and vehicle.

In order to collect the duty cycle and safety-related data, ORNL developed a data acquisition and wireless communication system that was placed on each test vehicle. Each signal recorded in this FOT was collected by means of one of the instruments incorporated into each data acquisition system (DAS). Native signals were obtained directly from the vehicle's J1939 and J1708 data buses. A VBOX II Lite collected information available from a Global Positioning System (GPS) including speed, acceleration, and spatial location information at a rate of $5 \mathrm{~Hz}$, and communicated this data via the CAN (J1939) protocol. The Air-Weigh LoadMaxx, a self-weighing system which determines the vehicle's gross weight by means of pressure transducers and posts the weight to the vehicle's J1939 data bus, was used to collect vehicle payload information. A cellular modem, the Raven X EVDO V4221, facilitated the communication between the eDAQ-lite (the data collection engine of the system) and the user, via the internet. The modem functioned as a wireless gateway, allowing data retrievals and system checks to be performed remotely. Also, and in partnership with FMCSA, two additional safety sensors were installed 
on the combination vehicles: the MGM e-Stroke brake monitoring system and the Tire SafeGuard tire pressure monitoring system. Both of these systems posted data to the J1939 data bus, enabling these signals to be read without any additional DAS interface hardware.

Seventy-three signals from the different deployed sensors and available vehicle systems were collected. Because of the differences in vehicle data buses (i.e., J1939 and J1708 data buses), not all desired signals were available for both types of vehicles. The safety-related sensors (i.e., the e-stroke device by MGM Brake and the Tire SafeGuard tire pressure monitoring system) post the signals to the J1939 data bus and were read as vehicle-native sensors similar to those provided by the vehicle on-board computer. The additional sensors, including the GPS-based VBOX II Lite, the self-weighing Air-Weigh unit, and a wiper switch used to collect basic rain data, were incorporated directly into the DAS.

Upon installation of the DAS, the sensors were individually monitored through a computer with a wired connection to the eDAQ-lite. When the proper operation of the installed sensors was confirmed, the cellular modem was connected and the ability to communicate with the system remotely via the internet was checked. When all systems appeared to be functioning as intended, the vehicle was released to resume normal operations. In addition, ORNL developed a data-retrieval and archiving system that accessed the vehicles automatically over the air and downloaded the information collected and residing on the on-board DAS. Each day the system e-mailed to the ORNL researchers a summary of the data downloaded from each vehicle, highlighting any sensors that showed a percentage of errors above a predefined threshold.

Data collection began in May 2009 with the official signing of the Memoranda of Agreement taking place at a ceremony held at the National Transportation Research Center in Knoxville, Tennessee on July 7 , 2009. During the one-year data collection period, the six participating vehicles logged over 95,000 miles (45,400 for the combination trucks and 49,400 for the transit buses) and consumed over 17,000 gallons of fuel $(6,000$ for the combination trucks and 11,300 for the transit buses), while conducting business in the East Tennessee area.

For the combination trucks, the largest proportion of idling time $(61 \%)$ and fuel consumed (50\%) while idling correspond to idling intervals that lasted between 0 and 5 minutes, that is, traffic congestion and delays at traffic signals. This is followed by intervals of 5 to 10 minutes of idling time $(25 \%$ of total idling time and $25 \%$ of total fuel consumed), and by the 15 to 60 minute time interval. The transit buses also spent most of their idling time (31\%) in congestion, and bus dwelling stops ( 0 to 5 minute idling interval) which also consumes the largest proportion of fuel spent while idling. However, as opposed to the combination trucks, the transit buses spent $26 \%$ ( $0 \%$ for the combination trucks) of their idling time in intervals that are larger than 4 hours consuming also about $26 \%$ of the fuel spent while idling. These large idling times were observed mostly at the KAT parking lot while the vehicles were waiting to start a trip.

For the transit buses, the percentage difference between the overall fuel efficiencies (i.e., fuel efficiencies computed taking into consideration idling times) and the moving fuel efficiencies (i.e., fuel consumed only when the vehicle was moving) was much higher (between 10 and 20 times higher) than that of the combination trucks. Some of this difference can be attributed to differences between vehicle types, but most is due to idling. While the total miles traveled by the two sets of vehicles during the year-long project was almost the same, the transit buses consumed almost 20 times more fuel than the combination trucks while idling due to the greater idling times.

One very important variable affecting the fuel efficiency of any vehicle is its payload level. ORNL developed a model that predicted the trailer weight based on the tractor weight provided by the Air-Weigh device (trailers were not instrumented with this device, so no direct measurements of trailer payload were 
made). On the other hand, the deployed Air-Weigh devices provided weight information for the entire vehicle in the case of the transit buses.

On average, the combination trucks weighted between $27,700 \mathrm{lbs}$ to $29,000 \mathrm{lbs}$ and the buses between 23,000 lbs and 23,800 lbs. To generate the distribution of fuel efficiency under the payload levels described above, 10-mile segments were considered for which the fuel efficiency was computed and counted as one observation. Overall, and as expected, the fuel efficiency decreases as the payload increases for the combination trucks. However, because the payload categories are very narrow and low (those combination trucks never made any trips with gross vehicle weights that were above 42,000 lbs, far below the legal weight limit for Class-7 trucks), the variation of the fuel efficiency is not significant. In the case of the transit buses, the relationship between fuel efficiency and vehicle weight is not as it would be predicted (i.e., decreasing fuel efficiency with increasing payload). This phenomenon is due to several factors. First, when the vehicle is empty (lowest payload) it spends a considerable amount of time idling. This results in very low fuel efficiencies for this load-level category. At the other end of the spectrum, the highway routes (express routes) are the ones that carry the largest number of passengers. The buses on these routes spent very little dwelling time (there are fewer bus stops) and also encounter the least number of traffic lights. This results in fuel efficiencies that are higher than regular surface street routes (light fare) with many more stops (bus stops and traffic signals).

The data collected in this project was also used to investigate the variability that may exist in duty cycles that are generated by the same vocation and that follow the same route. In order to do so, two sets of duty cycles collected from the data gathered by the transit buses were identified. The first set focused on freeway duty cycles. A $19 \mathrm{~km}$ segment of Westbound I-40 in Knoxville, Tennessee was selected and the corresponding duty cycles extracted from the database. The length of the 74 highway duty cycles ranged from 10.5 minutes to 27.6 minutes with an average length of 12.6 minutes and a standard deviation of 2.71 minutes. The variability of the duty cycles was measured by the ratio of the standard deviation of the distribution of duty cycle lengths over the mean of that distribution. For these 74 freeway duty cycles, this ratio was 0.214 (i.e., 2.71 minutes/12.7 minutes).

A $4.9 \mathrm{~km}$ loop in downtown Knoxville, Tennessee was selected and the corresponding duty cycles extracted from the database. Thirty-eight surface street duty cycles with the characteristics described above were selected. The length of the duty cycles ranged from 10.9 minutes to 22.8 minutes with an average length of 18.4 minutes and a standard deviation of 3.29 minutes. Computing the duty cycle variability in the same way as for the freeway duty cycles, the 38 surface street duty cycles had a ratio equal to 0.179 (i.e., 3.29 minutes/18.4 minutes). Under this variability measure, the highway duty cycles presented a higher variability than the surface street duty cycles. This higher variability in highway duty cycles is due to the effect of the variability in traffic conditions (i.e., highway congestion level). On surface-street routes, the main factors contributing to the duty cycle length are the number of bus stops and dwelling times (none of these factors are present on the highway duty cycles) with traffic congestion playing a relatively less preponderant role. 


\section{PROJECT OVERVIEW}

Since the early part of the 20th century, the US trucking industry has provided a safe and economical means of moving commodities across the country. Now, in the early part of the 21 st century, nearly $80 \%$ of the US domestic freight movement involves the use of trucks. The 2002 Vehicle Inventory and Use Survey (VIUS, Department of Commerce, 2005) estimates that there are over 3 million medium trucks [Class-3 through Class-7 trucks, between 10,001 and 33,000 lbs gross vehicle weight rating] registered in the US. It is estimated that Class- 6 and Class- 7 commercial vehicles together consume in excess of 4 billion gallons of fuel each year, second only to Class- 8 tractor-trailers in the commercial truck sector. ${ }^{1}$ With the increasing cost of fuel, increased efforts need to be placed on advanced technologies and new research and development efforts that will significantly contribute to enhanced fuel efficiencies.

The US Department of Energy (DOE) is spearheading a number of research efforts to improve heavy vehicle fuel efficiencies. This includes research in engine technologies (including hybrid and fuel cell technologies), lightweight materials, advanced fuels, and parasitic loss reductions. In addition, DOE is developing advanced tools and models to support heavy vehicle truck research, and is leading the 21st Century Truck Partnership (21CTP) $)^{2}$ whose stretch goals involve a reduction by $50 \%$ of the fuel consumption of heavy vehicles on a ton-mile basis.

This Medium Truck Duty Cycle (MTDC) Project is a critical element in DOE's vision for improved heavy vehicle energy efficiency. It involves the collection of real-world data for various situational characteristics (rural/urban, freeway/arterial, congested/free-flowing, good/bad weather, etc.) and looks at the unique nature of medium trucks' drive cycles (stop-and-go delivery, power takeoff, idle time, shortradius trips), to provide a rich source of data that can contribute to the development of new tools, provide DOE a sound basis upon which to make technology investment decisions, and provide a national archive of real-world-based medium-truck operational data to support heavy vehicle energy efficiency research.

A quantitative profile of the driving behavior of medium trucks does not currently exist. A thorough understanding of the operation of medium trucks within duty cycles that reflect real-world conditions is an asset that would have great benefit to DOE, other federal agencies (e.g., the US Department of Transportation (DOT), and their interest in truck safety; the Environmental Protection Agency (EPA), and their interest in real-time emissions data), and the overall trucking industry (e.g., to better understand how improvement in energy efficiencies can contribute toward improved profit margins).

\subsection{PRIOR DUTY CYCLE EFFORTS}

The Oak Ridge National Laboratory's (ORNL's) Center for Transportation Research (CTA) recently conducted the Heavy Truck Duty Cycle (HTDC) project which focused on the collection of duty cycle data relative to Class- 8 vehicles. ${ }^{3}$ The HTDC effort was conducted in three Phases.

Phase 1 of the HTDC project was conducted from January 2005 through March, 2006, and involved pilot testing of one instrumented Class-8 tractor-trailer, operating cross-country (East-to-West and North-toSouth) while fully loaded. The purpose of the pilot testing was to design an appropriate data collection suite and to field harden associated sensors, instrumentation and a data acquisition system (DAS). The effort involved outfitting a truck with sensors and instrumentation to collect 104 channels of data over a

1 Based on estimates from the 2002 VIUS.

2 http://www1.eere.energy.gov/vehiclesandfuels/about/partnerships/21centurytruck/

3 Capps G., O. Franzese, B. Knee, M. B. Lascurain, and P. Otaduy, Class-8 Heavy Truck Duty Cycle Project Final

Report (ORNL/TM-2008/122), 2008. 
three-month time frame. The truck completed two east-west runs and two north-south runs. In order to leverage the resources required for pilot testing, partnering was engaged in with Dana Corp. (Dana) of Kalamazoo, Michigan, and Michelin Americas Research and Development Corp. (MARC) of Greenville, South Carolina. Both Dana and MARC were interested in collecting data for their own research from the pilot testing and provided in-kind resources (e.g., gratis usage of a Dana tractor and trailer and specialized test equipment). MARC's interest involved the field testing of their New-Generation Wide-Based Single Tires (NGWBSTs) and standard dual tires to assess any fuel efficiency impacts. The pilot test involved alternating between standard dual tires and the NGWBSTs for the various runs. Over 50 gigabytes of data were collected during the pilot test.

Phase 2 of the HTDC project involved the design of a streamlined DAS and sensor suite that could withstand the rigors of being mounted on a commercial vehicle during its normal vocation, and would require no human interaction for initialization on vehicle startup at the beginning of each day or at the beginning of each route. Additionally, the number of major system components was reduced to four (DAS; vehicle self-weighing system; weather station; vehicle position and motion system). The resulting DAS was capable of collecting 60 channels of data and storing it internally in its dynamic memory. Each Phase 2 DAS went through a "burn-in" cycle for 30 days prior to being installed onto the test vehicles. A working fleet (Schrader Trucking of Jefferson City, Tennessee) was down-selected for partnership in the project, and six tractors and ten trailers were instrumented in the fall of 2006 using the HTDC DASs. The first vehicle to be instrumented was closely monitored in the field for 30 days for issues with the equipment, installation, driver distraction, and software. A Memorandum of Understanding between DOE, Schrader Trucking and ORNL was signed in late-October 2006, and all test vehicles were deployed in the field operational test (FOT) by mid-November 2006.

Phase 3 of the HTDC project involved conducting FOT, collecting and storing the data, developing the HTDC Duty Cycle Generation Tool (DCGenT), support of Powertrain Systems Analysis Toolkit module development, and data analysis. The FOT was completed in November 2007. The six instrumented heavy trucks and ten instrumented trailers cumulatively hauled freight for more than 690,000 miles in an area approximately 1,000 miles in diameter, centered near Jefferson City, Tennessee. During the FOT, 60 channels of data were collected on each test truck for a twelve-month period, resulting in the collection of 290 GB of raw data. The Phase 3 FOT is believed to be the most comprehensive real-world data for Class-8 long-haul performance known to exist. The data collected during the FOT included speed, fuel consumption, road grade, location, and weather conditions; and it is expected to be of value in truck fuel economy studies as called for in the Energy Independence and Security Act of 2007, and to EPA in their efforts to define a standardized duty cycle for Class- 8 trucks. The data analysis of the FOT data permitted the computation of fuel efficiencies for Class- 8 trucks as a function of the type of tires mounted on the tractor and trailer. These calculations, which were made using fuel consumption information obtained from the vehicles' data bus, showed an overall fuel efficiency above $6.0 \mathrm{mpg}$. This level of fuel efficiency is on the upper limits of today's large-truck fleets and is mostly a result of the participating trucking company partner being a very technologically-minded organization, providing excellent programs in driver training, and their extensive vehicle maintenance program (including, for example, maintaining constant tire pressures). The results of the data analysis showed that there is always a statistically significant improvement in fuel efficiency with respect to the base case (i.e., dual tires on both the tractor and trailer; duals-duals) when NGWBSTs are used on the tractor and/or trailer.

Moreover, the fuel efficiency improvement increases as the number of NGWBSTs on the truck increases, with observed improvements of around 6\% when either the tractor or the trailer was equipped with NGWBSTs, and more than $9 \%$ when both were mounted with these types of tires. When the data was parsed by tractor-trailer tire configuration and load level, the results of the analysis show that there was again an improvement in fuel efficiency with respect to the base case (i.e., duals-duals) when NGWBSTs are used. In fact, for the particular case in which all tires are NGWBSTs, there was considerable improvement in fuel efficiency with respect to the base case (i.e., improvements that were above 10\%), 
and those improvements were more pronounced as the load level increased. They were also statistically significant at the $99 \%$ level of confidence.

\subsection{OVERALL PROJECT DESCRIPTION}

This project involves efforts to collect, analyze and archive data related to medium-truck operations in real-world driving environments. Such data and information will be useful to support technology evaluation efforts and to provide a means of accounting for real-world driving performance within medium-class truck analyses. The project is being led by ORNL's CTA and involves private industry partners from various truck vocations.

The MTDC project is unique in that there currently does not exist a national database of characteristic duty cycles for medium trucks. This project involves the collection of data from multiple vocations (four vocations) and multiple vehicles within these vocations (three vehicles per vocation) while the vehicles conduct their normal operations. The collection of this data is does not perturb the vehicle's normal duty cycle. For the MTDC Part 1 FOT, three vehicles, each from two vocations (urban transit and dry-box delivery) were instrumented for one year of data collection. The MTDC Part 2 FOT will involve the towing/recovery and utility vocations.

This program involves a number of tasks to collect and analyze real-world duty cycle data from medium trucks data in order to accomplish the following objectives:

- Provide a source of real-world medium-truck performance data that can be utilized by DOE for making decisions related to future technology investments.

- Provide a baseline of data that can be utilized to gauge 21CTP technology advancements.

- Provide a national source of real-world data for the medium-truck research community.

- Potentially provide data germane to EPA's goal of collecting emissions data from medium trucks in quantifiable driving environments.

- Potentially provide data germane to the Federal Motor Carrier Safety Administration's (FMCSA's) goal of collecting vehicle, driver and carrier data in real-time during normal vocational operation.

\subsection{PARTNERSHIPS}

The vehicles participating in the MTDC project are doing so through gratis partnerships in return for access to the results of this study. Partnerships such as these are critical to FOTs in which real-world data are being collected. A partnership with FMCSA allowed the collection of additional safety-related signals beyond the normal set of duty cycle data collected in previous efforts.

\subsubsection{H.T. Hackney}

The H.T. Hackney Company ${ }^{4}$ is one of the largest wholesale distributors in the country, distributing products to 21 states. ORNL worked with personnel at their Roane County facility to collect real-world duty cycle data on three of their Class-7 units whose duty cycles involved delivery to local convenience stores. This fleet's safety consciousness and progressive attitude toward fuel efficiency also made them an excellent venue for the installation of additional safety sensors to supplement the standard duty cycle data.

4 http://www.hthackney.com/ 


\subsubsection{K-Trans Management}

K-Trans Management operates the Knoxville Area Transit (KAT) ${ }^{5}$, the City of Knoxville's transit system, operating services across the city. They partnered with ORNL to provide access to three of their Class-7 buses for collection of duty cycle data.

\subsubsection{Federal Motor Carrier Safety Administration}

Additionally, ORNL has collaborated with FMCSA to determine if there were possible synergies between the MTDC duty cycle data collection effort and FMCSA's need to learn more about the operation and duty cycles of the second-largest fuel consuming commercial vehicle category in the US. FMCSA's primary interest was in collecting safety data relative to the driver, carrier, and vehicle. Further, FMCSA is in the process of developing and testing requirements for wireless transfer of data from a commercial vehicle to the roadside.

Beyond the standard duty cycle data collection system used in previous DOE duty-cycle research, additional sensors were installed on three test vehicles (Class-7 single-axle, tractor-trailers) to collect several safety-related signals of interest to FMCSA. These additional sensors were provided through a partnership with FMCSA who provided for the additional cost of incorporating these sensors into the DAS. The real-time brake stroke, tire pressure, and weight information obtained from these sensors makes possible a number of safety-related analyses such as determining the frequency and severity of braking events and tracking tire pressure changes over time.

5 http://www.katbus.com/ 


\section{PART 1 FIELD OPERATIONAL TEST}

For the MTDC Part 1 FOT, three vehicles each from two vocations (urban transit and dry-box delivery) were instrumented for one year of data collection (2009-2010). The Part 2 MTDC FOT will involve the towing/recovery and utility vocations, and data will be collected in the 2010-2011 timeframe.

\subsection{VOCATIONAL ASSESSMENT}

Within the medium weight category of trucks, there is such a variety of applications that it is virtually impossible to identify a single typical duty cycle for the weight class. Therefore, the decision was made to select a few major medium-truck vocations for the MTDC FOT. A vocational assessment ${ }^{6}$ was conducted to identify several recommended vocations for participation in the FOT for the MTDC project.

\subsubsection{Initial Down-Selection}

The following vocations were originally under consideration for participation in the FOT based on available VIUS data:

- Dry-box delivery

- Beverage delivery

- Refrigerated/frozen delivery

- Package delivery

- Utility (where power takeoff [PTO] is used)

- Public transit

- School bus

- Towing and recovery

- Farming

Because of the limited number of DASs available and the desire to collect seasonal data, not all of these vocations could participate in the FOT. The list of vocations was down-selected to six vocations. The selections were made to give a typical representation of medium trucks based on vehicle miles traveled, hours of operation per day, typical payload, area of operation, PTO operation, auxiliary cargo heating and cooling, and daily idle time.

Farming vehicles were removed from the list because most medium trucks used in farming operations are not over-the-road vehicles in regular use. An additional vocation which was considered but not chosen for participation in the FOT was package delivery. This vocation was not selected because although it may be distinct from other vocations, the data collected would be inapplicable to all but the very few companies in this industry. It was therefore omitted from the final list due to the limited application of this duty cycle data; there are very few entities that would find this duty cycle characteristic of their operations.

The rental truck vocation was also considered because it represents a significant number of medium trucks-particularly Class-6 trucks. However, in addition to the "niche market" reason for excluding package delivery, equipment security was also an issue. Unlike other vocations where the drivers are employed by the company, the drivers of these vehicles could not be known and therefore presented a potential data security risk and risk of damage to or theft of the data collection equipment.

6 Internal ORNL report 
Another vocation under consideration was beverage delivery. However, when the potential partner proved unable to participate, it was decided that the duty cycle of this vocation may be very similar to that of refrigerated/frozen delivery and dry-box delivery. Thus, a replacement for this fleet was not pursued.

\subsubsection{Recommended Vocations}

Following the formal down-selection, the following vocations were recommended for the FOT.

\subsubsection{Dry Box Delivery}

The dry box delivery trucks are expected to be in operation the entire business day and travel routes within a certain distance from the distribution center. The payload of these vehicles is expected to range from near-capacity to empty throughout the day as deliveries are made.

\subsubsection{Refrigerated/Frozen Delivery}

This vocation is expected to have a lower fuel efficiency than dry-box delivery due to the energy requirements of the refrigeration system. The trucks involved in this type of delivery will likely go out fully loaded on a regular basis. Of particular interest is the seasonal variation in the energy demands of the refrigeration system.

\subsubsection{Utility}

The duty cycles of utility trucks are unique in that the typical utility truck spends very little time traveling (and thus has a much lower annual mileage than other medium trucks). These trucks typically spend most of their operating time in PTO operation, up to eight hours in a ten-hour operational day. It is expected that data collected from the utility trucks will reveal negligible change in weight. These vehicles travel in both rural and urban areas.

\subsubsection{Urban Transit}

Urban transit buses experience stop-and-go operations throughout the day, often driving in congested areas. Although these vehicles are expected to drive predictable routes, the collection of data for an entire twelve-month period will permit seasonal data to be collected.

\subsubsection{Rural Pupil Transportation}

The inclusion of a rural school bus will permit the collection of an exclusively rural duty cycle. Unlike other vocations selected, there will be times during the year when these vehicles are not in operation. This duty cycle is unique because although it is based on a regular schedule, it does not typically include hours of operation during the entire business day.

\subsubsection{Towing and Recovery}

Unlike the other vocations selected, vehicles employed in towing and recovery do not have regular, predictable duty cycles. Furthermore, the payload will likely be either fully loaded or unloaded. Like the utility trucks, these vehicles are expected to travel in both urban and rural areas. 


\subsubsection{Selected Vocations}

Based on further correspondence with the project sponsor, the list of vocations to be tested was reduced to the following four vocations.

- Local delivery

- Urban transit

- Towing and recovery

- Utility

The selected test methodology allows a full year of duty cycle data to be collected from three vehicles from each vocation. The first two vocations were involved in the Part 1 FOT; the Part 2 FOT will involve the final two vocations with an emphasis on PTO operation.

\subsection{DATA ACQUISITION SYSTEM AND SENSORS}

A DAS was placed on each test vehicle to collect relevant duty cycle data for wireless upload and subsequent analysis. Native signals were obtained directly from the vehicle's J1939 and J1708 data buses. The DAS enclosure is shown in Figure 1.

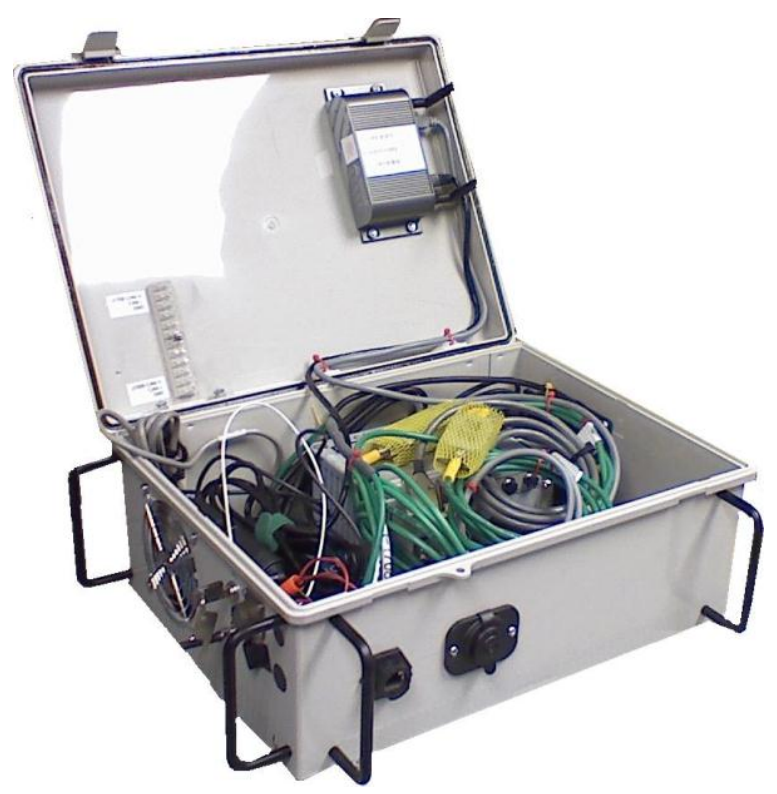

Figure 1. MTDC DAS

The VBOX II Lite (Figure 2) collected Global Positioning System (GPS)-related information including speed, acceleration, and GPS at a rate of $5 \mathrm{~Hz}$. This data was communicated via the CAN (J1939) protocol. 


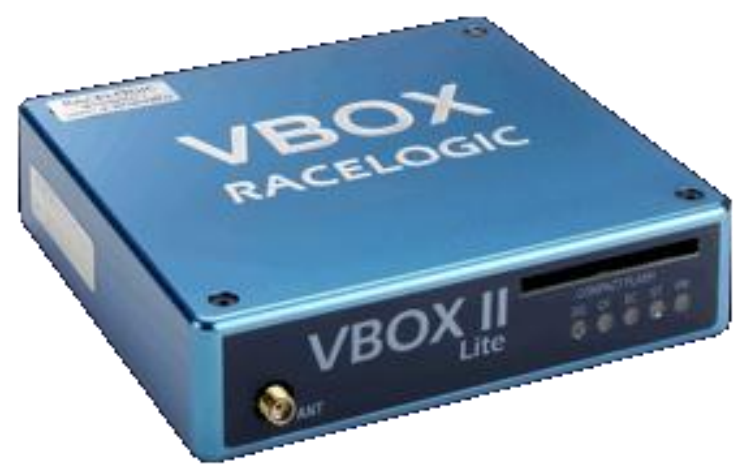

Figure 2. VBOX II Lite GPS Unit

The Air-Weigh LoadMaxx (Figure 3) is a self-weighing system which determines the vehicle's gross weight by means of pressure transducers and posts the weight to the vehicle's J1939 data bus.

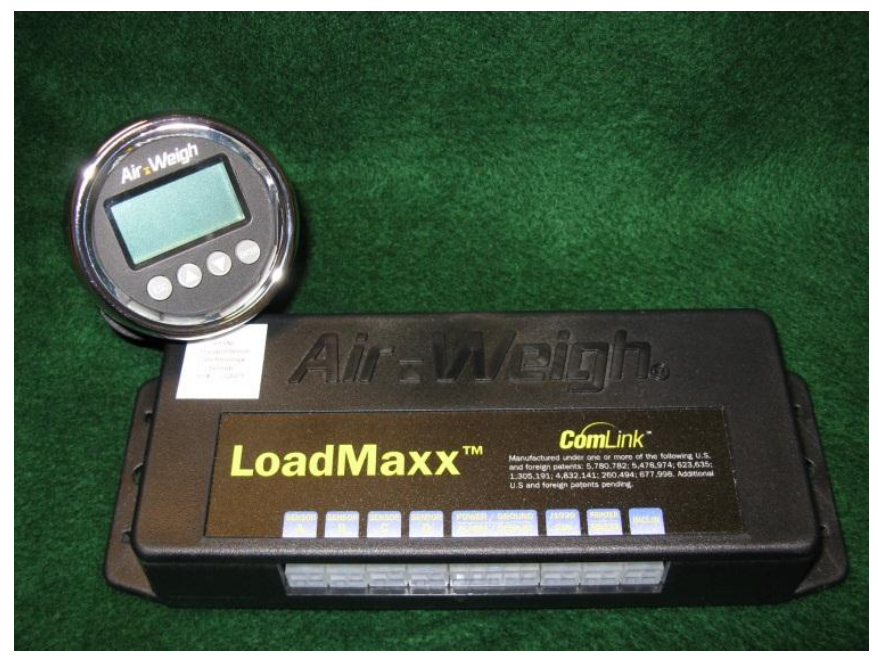

Figure 3. Air-Weigh LoadMaxx Kit

The eDAQ-lite data acquisition unit (Figure 4) collected and stored data from the vehicle's J1939 and J1708 data bus and connected J1939 sensors (i.e., the VBOX and Air-Weigh units). For the purposes of the FOT, it was configured to record all data at a rate of $5 \mathrm{~Hz}$. The eDAQ-lite was the central data collection unit with internal data storage for use between periodic uploads. 


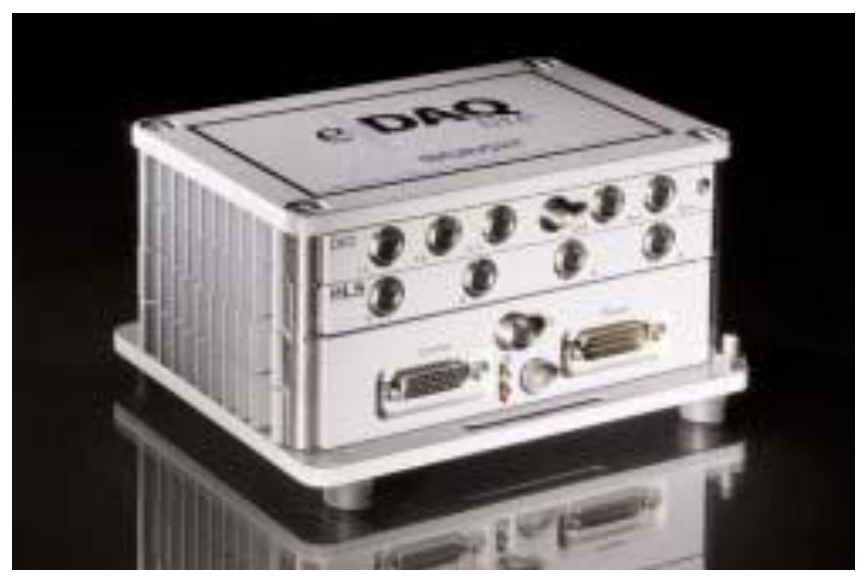

Figure 4. EDAQ-lite Data Acquisition Unit

The Raven X EVDO V4221 (Figure 5) is the cellular modem which facilitated the communication between the eDAQ-lite and the user via the internet. The modem functioned as a wireless gateway, allowing data retrievals and system checks to be performed remotely.

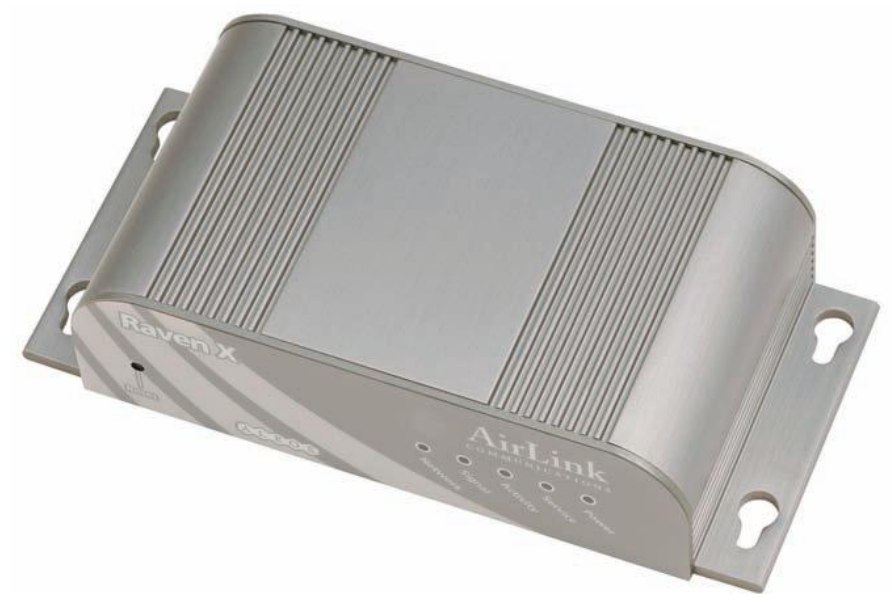

Figure 5. Raven X EVDO Cellular Modem

In partnership with FMCSA, two additional safety sensors were installed on the combination vehicles: the MGM e-Stroke brake monitoring system (Figure 6) and the Tire SafeGuard tire pressure monitoring system (Figure 7). Both of these systems posted data to the J1939 data bus, enabling these signals to be read without any additional DAS interface hardware.

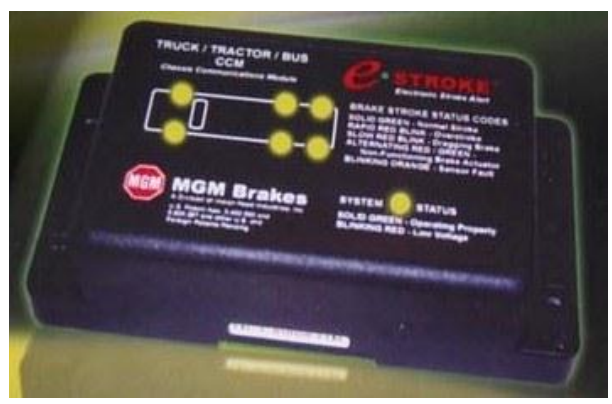

Figure 6. MGM E-Stroke Control Unit 


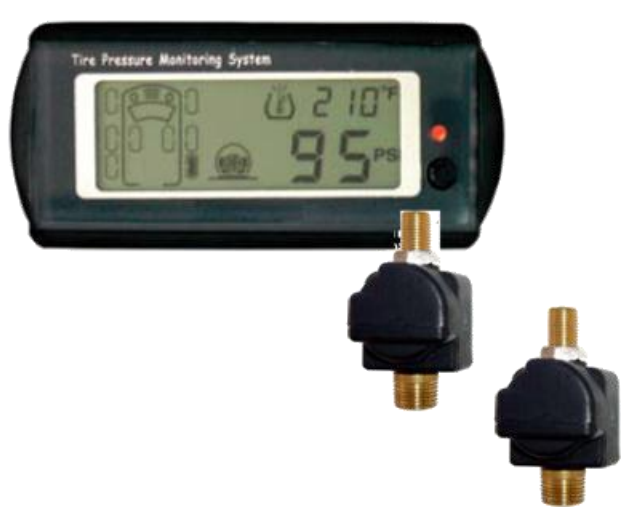

Figure 7. Tire SafeGuard Display and Transducers

\subsection{TEST VEHICLES}

For Part 1 of the MTDC FOT, three vehicles each from two vocations, Class- 7 combination tractor-trailer (tractor-trailer) and urban transit bus, were instrumented with the previously-constructed HTDC DASs to collect 12 months of real-world data. Vehicles from the remaining vocations (utility and towing/recovery) will be instrumented with the DASs in Part 2 of the MTDC FOT.

Medium tractor-trailers were represented by the local delivery vocation. The H.T. Hackney Company made available three 2005/2007 International day-cab tractors, model 8600, which regularly haul 28-ft pup trailers. These vehicles have routes within a 60-80 mile radius of H.T. Hackney's Roane County facility. The trailers have three compartments: insulated, refrigerated, and frozen. Because the cooling for the trailer is powered separately (i.e., a separate diesel engine run from a separate fuel tank located on the trailer), there will be no additional parasitic losses (which would influence fuel efficiency) due to the refrigerated units. These vehicles' regular daily operation involves delivery of 16,000 to 20,000 lbs of cargo, while generally covering 125 miles on a given day. A typical H.T. Hackney vehicle is pictured in Figure 8. In addition to the standard data acquisition equipment, the tractors were up-fitted with additional safety technologies provided by FMCSA. 


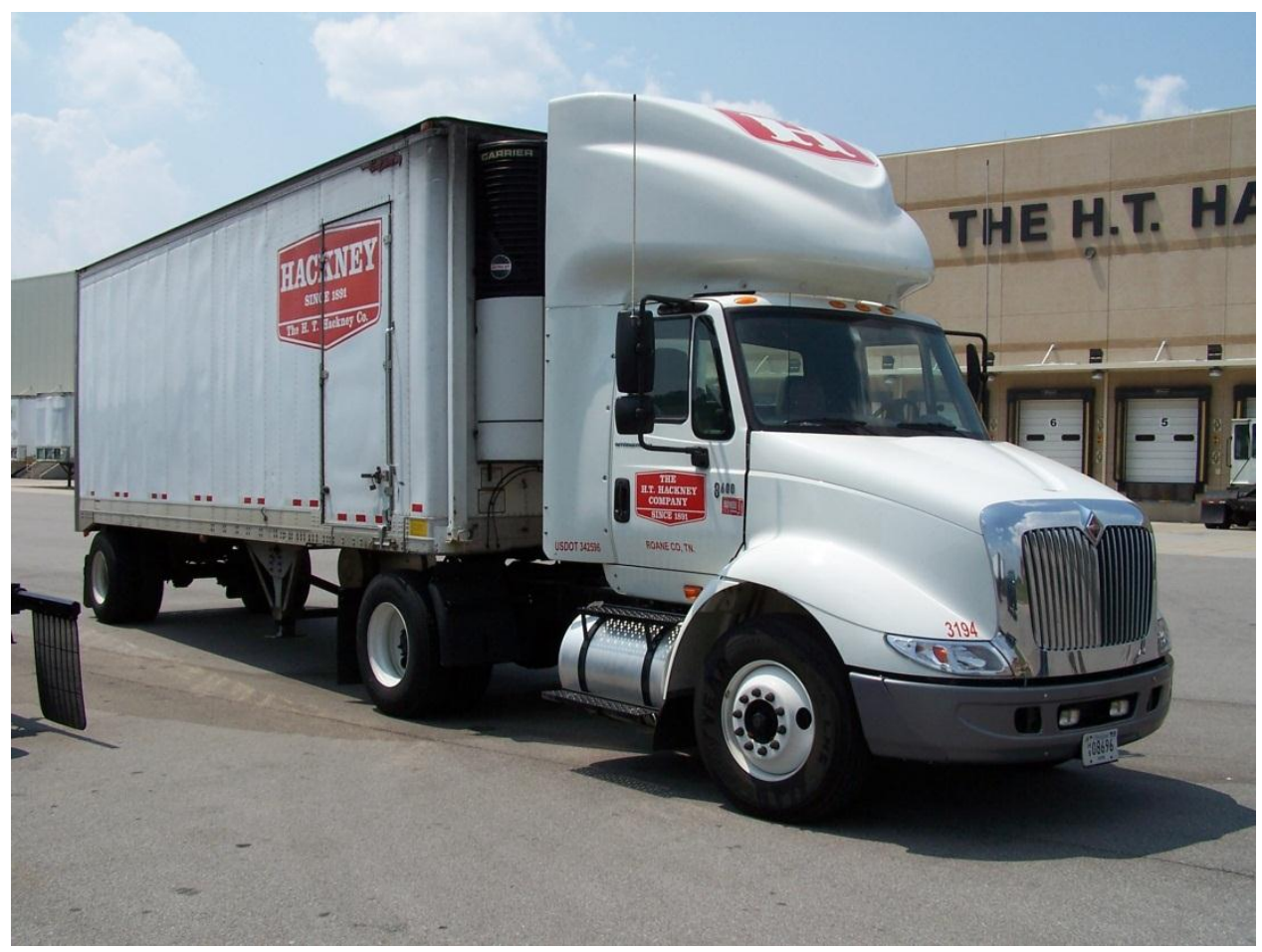

Figure 8. H.T. Hackney 2007 International 8600

The transit buses were made available for the Part 1 MTDC FOT by K-Trans Management which operates KAT. These vehicles are 2005 Optima LF-34 buses. While additional safety technologies were not installed on these vehicles, the standard DAS and Air-Weigh self-weighing units were used. These vehicles' regular operation involves short trips throughout the Knoxville area; although the majority of the routes traveled by these buses consist of surface streets, highway data was also collected. A typical test vehicle is shown in Figure 9. 


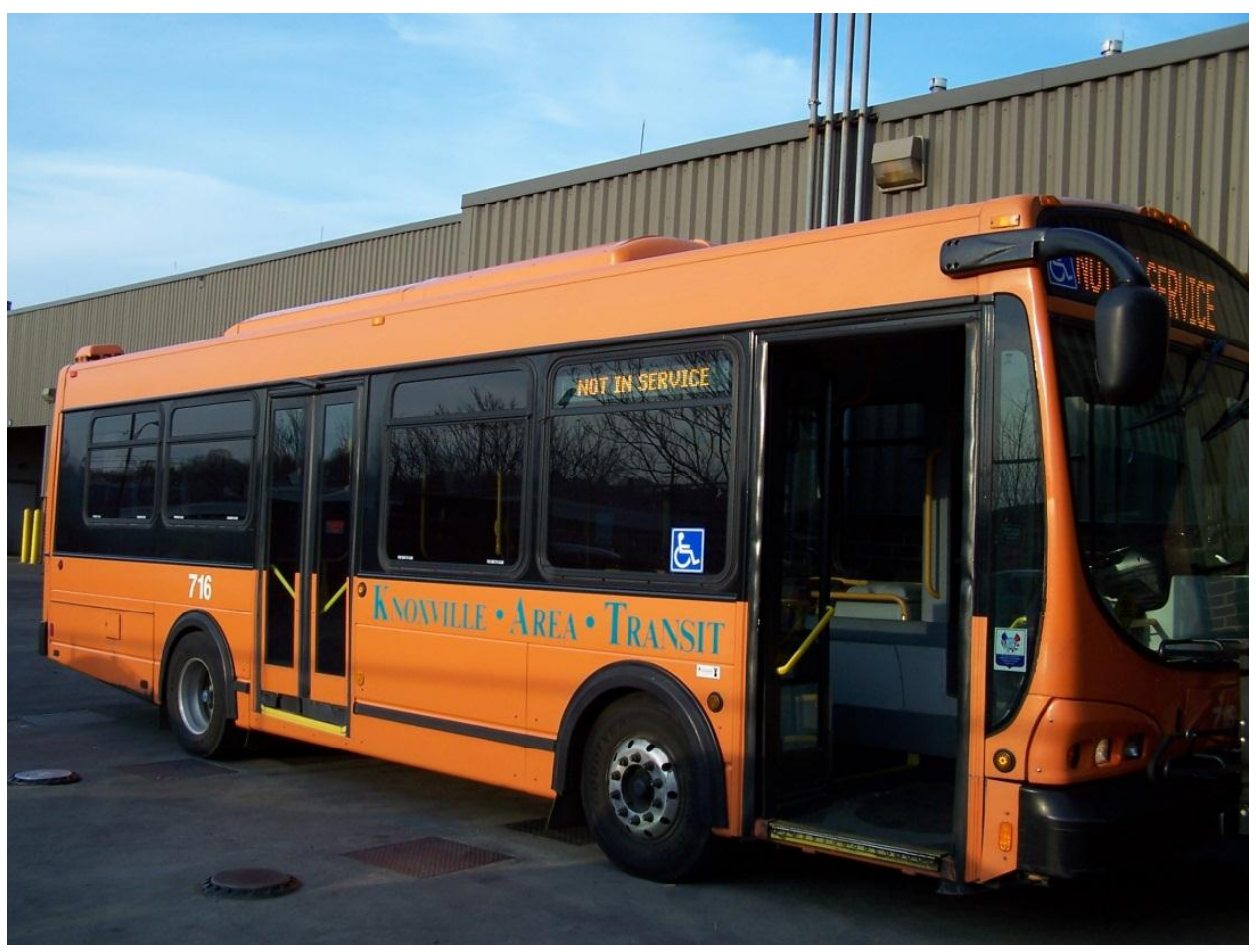

Figure 9. KAT Transit Bus

\subsection{COLLECTED SIGNALS}

Because of the differences in vehicle data buses, not all desired signals were available for both vehicles. Table 1 shows the list of signals and their availability on the tractors' and urban transit buses' J1939 and J1708 data buses. The signals available for each vocation are similar, but may not be identical where different data buses are used. For example, the J1939 data bus signals are often updated more frequently than their J1708 counterparts. Typically, SI units are used for J1939 data, whereas the J1708 signals are occasionally reported in US Customary Units.

For a vehicle with a J1939 data bus, the torque values reported by the data bus are in terms of percent of a reference value. This "Engine Reference Torque" is a static value which is valid as long as the engine is not re-programmed with a different engine map (which is rarely done). This value can therefore be read from the data bus once for use in interpreting duty cycle data. During the test vehicle assessment, this value was found to be $2,118 \mathrm{Nm}$ for the delivery vehicle tested and 1,024 $\mathrm{Nm}$ for the transit bus. 
Table 1. Vehicle Data Bus Signals

\begin{tabular}{|c|c|c|c|}
\hline No. & Description & Delivery & $\begin{array}{l}\text { Urban } \\
\text { Transit }\end{array}$ \\
\hline 1 & Total Vehicle Distance & J1939 & J1939 \\
\hline 2 & Road Speed Limit Status & J1939 & J1939 \\
\hline 3 & Wheel-Based Vehicle Speed/Road Speed & J1939 & J1939 \\
\hline 4 & Front Axle Speed & J1939 & J1939 \\
\hline 5 & Engine Speed & J1939 & J1939 \\
\hline 6 & Current Gear & J1939 & $-\cdots$ \\
\hline 7 & Selected Gear & J1939 & J1939 \\
\hline 8 & Actual Gear Ratio & J1939 & $-\cdots$ \\
\hline 9 & Output Shaft Speed & J1939 & J1939 \\
\hline 10 & Transmission Selected Range & J1939 & $\mathrm{J} 1708$ \\
\hline 11 & Transmission Current Range & J1939 & $\mathrm{J} 1708$ \\
\hline 12 & Engine Oil Temperature & $\mathrm{J} 1939$ & --- \\
\hline 13 & Intake Manifold Temperature & J1939 & J1939 \\
\hline 14 & Engine Coolant Temperature & J1939 & J1939 \\
\hline 15 & Boost Pressure & $\mathrm{J} 1708$ & $\mathrm{~J} 1708$ \\
\hline 16 & Fuel Rate & J1939 & J1939 \\
\hline 17 & Instantaneous Fuel Economy & J1939 & $\mathrm{J} 1939$ \\
\hline 18 & Actual Engine - Percent Torque & J1939 & J1939 \\
\hline 19 & Percent Accelerator Pedal Position & J1939 & J1939 \\
\hline 20 & Percent Load at Current Speed & J1939 & J1939 \\
\hline 21 & Driver's Demand Engine - Percent Torque & J1939 & J1939 \\
\hline 22 & Nominal Friction Percent Torque & J1939 & $\mathrm{J} 1939$ \\
\hline 23 & Brake Switch & J1939 & J1939 \\
\hline 24 & Clutch Switch & J1939 & $-\cdots$ \\
\hline 25 & Cruise Control Accelerate Switch & $\mathrm{J} 1939$ & J1939 \\
\hline 26 & Cruise Control Active & J1939 & J1939 \\
\hline 27 & Cruise Control Coast Switch & $\mathrm{J} 1939$ & $\mathrm{~J} 1939$ \\
\hline 28 & Cruise Control Enable Switch & J1939 & J1939 \\
\hline 29 & Cruise Control Resume Switch & J1939 & J1939 \\
\hline 30 & Cruise Control Set Switch & $\mathrm{J} 1939$ & J1939 \\
\hline 31 & Cruise Control Set Speed & J1939 & J1939 \\
\hline 32 & Power Takeoff Governor/Status Flags & J1939 & J1939 \\
\hline 33 & Power Takeoff Set Speed & J1939 & J1939 \\
\hline 34 & Total Power Takeoff Hours & J1939 & J1939 \\
\hline 35 & Battery Voltage & J1939 & $\mathrm{J} 1939$ \\
\hline 6 & Fan Drive State & J1939 & J1939 \\
\hline 37 & AC High Pressure Fans Switch & $\mathrm{J} 1939$ & $\mathrm{~J} 1939$ \\
\hline 38 & Barometric Pressure & J1939 & J1939 \\
\hline
\end{tabular}

For the actual data collection, certain on/off status flags were incorporated into a single channel for more efficient use of data acquisition resources. For example, signals 23-24 were combined into a Brake/Clutch Switch channel, and signals 25-30 were combined into a Cruise Control Status channel. The individual indicators may be easily recovered via post-processing of the data. 
Table 2 lists additional signals available from sensors incorporated into the DAS. These additional sensors include the GPS-based VBOX II Lite and the self-weighing Air-Weigh unit. In order to collect basic rain data, a channel was used to monitor the wiper switch as well.

Table 2. Signals from Other Sensors (All Part 1 Vehicles)

\begin{tabular}{|c|l|c|}
\hline No. & \multicolumn{1}{|c|}{ Description } & Sensor \\
\hline 39 & Latitude & VBOX II Lite \\
\hline 40 & Longitude & VBOX II Lite \\
\hline 41 & Altitude & VBOX II Lite \\
\hline 42 & Vertical Velocity & VBOX II Lite \\
\hline 43 & Velocity over Ground & VBOX II Lite \\
\hline 44 & Longitudinal Acceleration & VBOX II Lite \\
\hline 45 & Lateral Acceleration & VBOX II Lite \\
\hline 46 & Heading & VBOX II Lite \\
\hline 47 & Satellites & VBOX II Lite \\
\hline 48 & Time UTC & VBOX II Lite \\
\hline 49 & Distance & VBOX II Lite \\
\hline 50 & Steer Axle Weight & Air-Weigh \\
\hline 51 & Drive Axle Weight & Air-Weigh \\
\hline 52 & Wiper Switch Status & Wiper Switch \\
\hline
\end{tabular}

In addition to the tentative signals already listed, additional safety-related sensors were provided via partnership with FMCSA. Two areas of interest to FMCSA are brake system operation and dynamic weight changes to vehicle components and tires during operation. The e-stroke device by MGM Brake and the Tire SafeGuard tire pressure monitoring system post the signals described in Table 3 to the J1939 data bus; these signals provided some of the data of interest to FMCSA related to brake operation and tire pressure. 
Table 3. Additional Safety Signals (Tractor of Delivery Vehicles Only)

\begin{tabular}{|c|l|c|}
\hline No. & \multicolumn{1}{|c|}{ Description } & Sensor \\
\hline 53 & Brake Actuator Status - Left Front & E-Stroke \\
\hline 54 & Brake Actuator Status - Right Front & E-Stroke \\
\hline 55 & Brake Actuator Status - Left Rear & E-Stroke \\
\hline 56 & Brake Actuator Status - Right Rear & E-Stroke \\
\hline 57 & Lining Status - Left Front & E-Stroke \\
\hline 58 & Lining Status - Right Front & E-Stroke \\
\hline 59 & Lining Status - Left Rear & E-Stroke \\
\hline 60 & Lining Status - Right Rear & E-Stroke \\
\hline 61 & Brake Application Pressure & E-Stroke \\
\hline 62 & Tire Pressure - Left Front & Tire SafeGuard \\
\hline 63 & Tire Pressure - Right Front & Tire SafeGuard \\
\hline 64 & Tire Pressure - Left Rear Outside & Tire SafeGuard \\
\hline 65 & Tire Pressure - Left Rear Inside & Tire SafeGuard \\
\hline 66 & Tire Pressure - Right Rear Inside & Tire SafeGuard \\
\hline 67 & Tire Pressure - Right Rear Outside & Tire SafeGuard \\
\hline 68 & Tire Temperature - Left Front & Tire SafeGuard \\
\hline 69 & Tire Temperature - Right Front & Tire SafeGuard \\
\hline 70 & Tire Temperature - Left Rear Outside & Tire SafeGuard \\
\hline 71 & Tire Temperature - Left Rear Inside & Tire SafeGuard \\
\hline 72 & Tire Temperature - Right Rear Inside & Tire SafeGuard \\
\hline 73 & Tire Temperature - Right Rear Outside & Tire SafeGuard \\
\hline
\end{tabular}

\subsection{INSTALLATION AND SHAKEDOWN TESTING}

\subsubsection{Physical Installation}

Instrumentation of the test vehicles involved making connections to the vehicle's internal wiring to tap into the data bus, to obtain power for the DAS, and to monitor the ignition switch. Cable runs to and from sensors were routed in a manner to allow for sustained testing and occupant safety. Consideration was also given to shielding these cables from engine/exhaust heat and damage from objects thrown from the roadway.

Prior to installation, ORNL staff discussed the location, orientation, and connections for the DAS with managers and technicians at the partner facilities to ensure that the equipment would be installed in such a way as to be "transparent" to the vehicle operator and not require driver interaction, while still permitting safe access by test engineers when necessary. This partner input also ensured that the installation would not damage the structure of the test vehicles, affect their appearance once the equipment is removed, or decrease the value of the vehicle beyond normal depreciation. ORNL provided the materials and accompanying instructions for installation of cabling and GPS antennas and installed the actual DASs on the partners' prepared vehicles. All equipment was securely fastened in place to prevent driver distraction and to avoid damage to the test equipment.

Installation and calibration of the self-weighing system was performed by Air-Weigh personnel. The calibrations of the Air-Weigh units were performed at the partners' respective bases of operations. Portable scales for the calibration were provided by the Tennessee Department of Safety via partnership with FMCSA. 


\subsubsection{Sensor/DAS Verification}

Upon installation of the DAS, the sensors were individually monitored through a computer with a wired connection to the eDAQ-lite. When the proper operation of the installed sensors was confirmed, the cellular modem was connected and the ability to communicate with the system remotely via the internet was checked. When all systems appeared to be functioning as intended, the vehicle was released to resume normal operations.

Prior to the start of the FOT, in order to diagnose any sensor and data problems which were not recognized during the equipment configuration stage, one test vehicle was instrumented for approximately one month of shakedown testing. During this time, minor adjustments and corrections were made to equipment settings, and frequent data uploads were performed. Procedures to be used throughout the FOT were finalized, and data analysis was performed on a small scale in order to identify problems in the analysis software so that they could be corrected before the full FOT. One goal of this preliminary testing was to verify that the use of a cellular modem to interface with the eDAQ-lite through the internet provided an efficient and robust data collection solution. At the successful conclusion of the shakedown testing, the remaining test vehicles were instrumented and deployed.

\subsection{CONDUCT OF THE FOT}

\subsubsection{Launch of the FOT and MOA Signing}

Data collection began in May 2009 with the official signing of the Memoranda of Agreement (MOA) taking place at a ceremony on July 7, 2009, at the National Transportation Research Center (NTRC, http://www.ntrc.gov/), located in Knoxville, Tennessee (Figure 10). Representatives from DOE, DOT, H.T. Hackney, and K-Trans Management participated in this event, which was well-attended by the media.

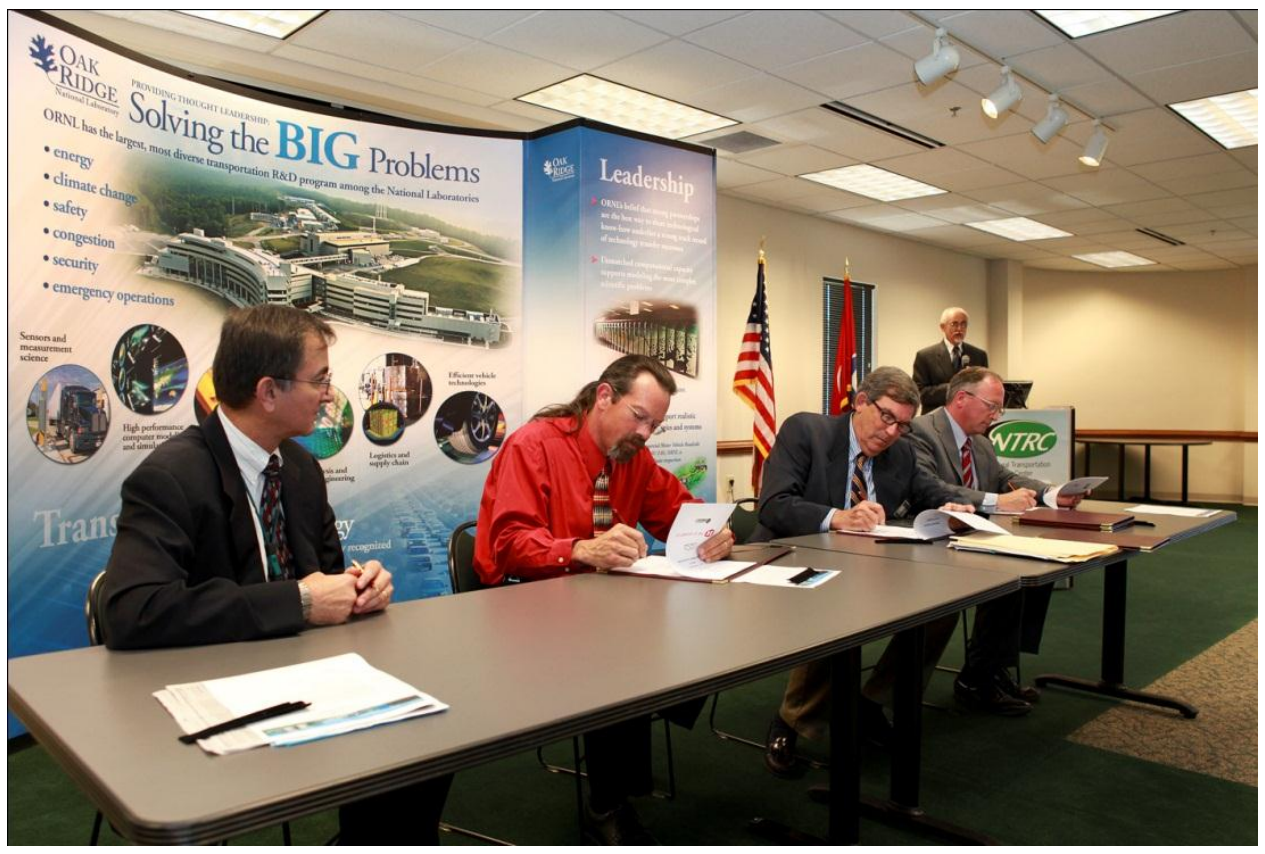

Figure 10. MOA Signing Ceremony (July 7, 2009) 


\subsubsection{Data Retrieval Software}

In order to efficiently collect data from test vehicles based in two different locations, cellular modems were integrated into each DAS. This methodology permitted test data to be uploaded via the internet, making regular site visits unnecessary for properly-functioning systems.

With the twelve-month FOT of six test vehicles, the uncompressed data files reached approximately 190 GB of raw data. Because of the large volume of data to be collected, regular data uploading, preliminary checking, and data archiving was performed throughout the FOT. This also permitted the recognition of sensor problems soon after development to minimize data loss due to equipment malfunctions.

The data retrieval process generated two copies of the data, stored on separate external hard drives on the project computer. An additional copy of these drives was kept on an ORNL server maintained for this purpose at the ORNL campus (located approximately 12 miles from the NTRC), backed up on a daily basis to protect against data loss. The retrieval and archiving process was fully automated and did not require human intervention. Each day, the system e-mailed to the ORNL researchers a summary of the data downloaded from each vehicle, highlighting any sensors that showed a percentage of errors above a pre-defined threshold. Any identified problem was quickly corrected either by accessing the data collection system over the air, or by physically accessing the system and making the necessary adjustments or replacements.

\subsection{DE-INSTRUMENTATION}

The DAS and sensor suites were removed from each of the test vehicles at the conclusion of the FOT. The majority of this de-instrumentation was performed by technicians at the partners' facilities. Because it was not cost-effective to remove the safety sensors (provided by FMCSA) from the combination test vehicles and re-install them for future projects, these sensors remained installed on those test vehicles for potential future research and use by H.T. Hackney in their normal operations. Additional cables for discrete sensor or systems were left in place at the partners' discretion. This decision saved DOE/ORNL the cost of removing the cabling from the test vehicle (Note: this type of cabling has no remaining value to DOE/ORNL once removed). The DAS and sensors were returned to ORNL for use in the Part 2 MTDC effort. The fleet vehicle was "restored" as needed after the equipment was removed (e.g., cable feed-through holes were plugged, dash cut-outs were covered, and electrical connections were terminated). 


\section{ANALYSIS OF THE PART 1 FOT DATA}

The main goal of the MTDC project was to collect real-world performance and spatial data related to the operations of Class- 6 and -7 vehicles from fleets engaged in normal commerce, and to provide a means for managing and using the collected data to generate real-world-based duty cycle data and information. In the Part 1 of this project, three Class-7 tractors from the H.T. Hackney fleet and three transit buses from KAT were instrumented and over 70 channels of data were collected for over a one-year period of time at a rate of $5 \mathrm{~Hz}$. The H.T. Hackney local fleet, which is headquartered in Roane County, Tennessee, operates within an East Tennessee region covering an area that extends from Cookeville, Tennessee to the border with North Carolina in the east-west direction, and from the Tennessee-Georgia border to the Tennessee-Kentucky border in the north-south direction (see Figure 11 for spatial coverage of the three trucks that participated in this project). The KAT operates in Knoxville, Tennessee, and surrounding areas; the routes of the buses participating in this project are shown in Figure 12.

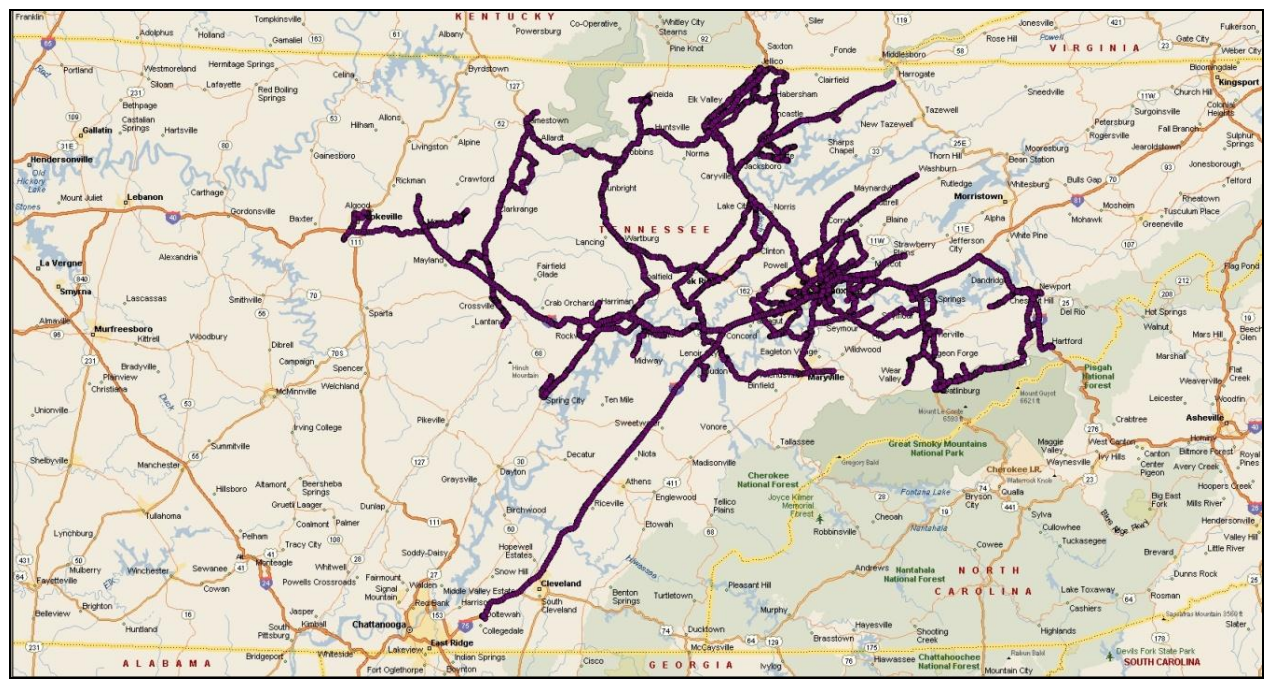

Figure 11. Routes of Participating H.T. Hackney Trucks (East Tennessee Area)

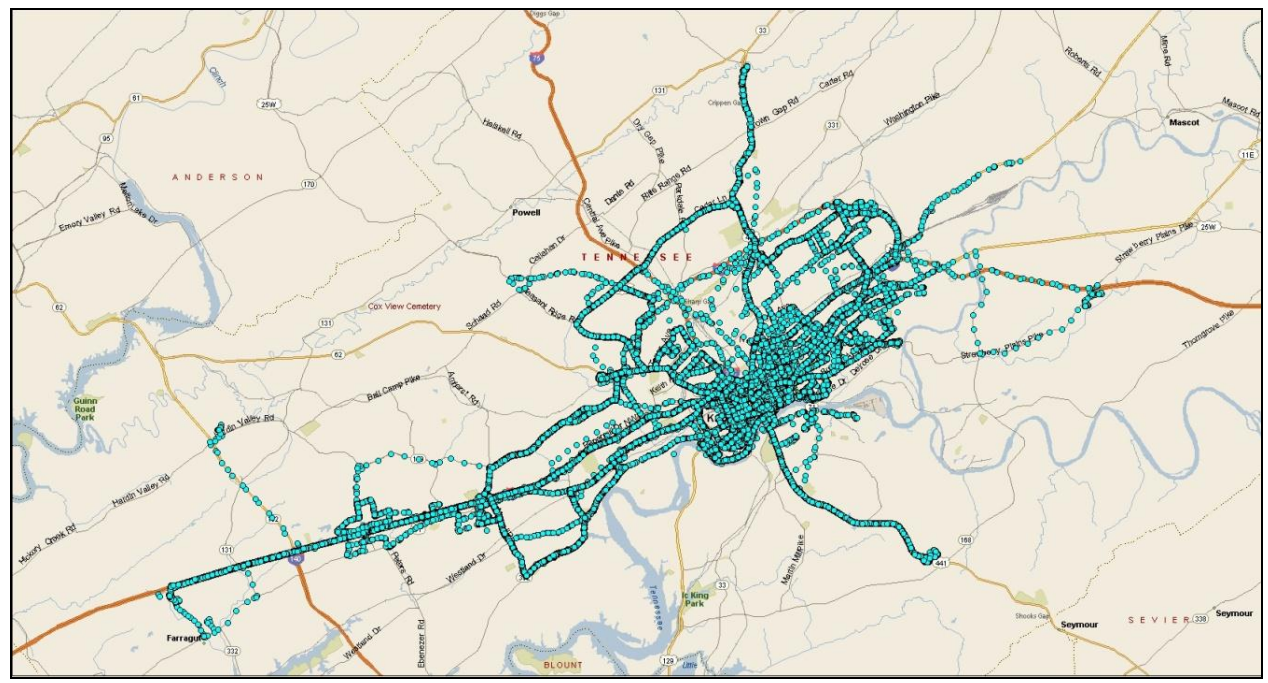

Figure 12. Routes of Participating KAT Buses (Knoxville, Tennessee Area) 
The data gathered in this project included information such as instantaneous fuel rate, engine speed, gear ratio, vehicle speed, and other information read from the vehicle's data bus; spatial information (latitude, longitude, altitude) acquired from a GPS device; and instantaneous tractor (for the combination trucks) and bus weight were obtained from devices mounted on the six participating vehicles. Some of the vehicles were also mounted with safety sensors providing brake and tire information. Over the duration of the Part 1 MTDC FOT (just over a year), the three combination trucks traveled nearly 45,500 miles, while the three buses logged 49,500 miles.

This section presents the first results of a data analysis of the information collected in the Part 1 MTDC FOT. Before the results are presented, the next section describes, in some detail, the data cleansing process and the organization of the extensive information collected in this project. Subsequently, a discussion is provided regarding additional models (such as a vehicle weight model) that were developed to complement the collected information. Those models were developed using the data gathered in the project and were used to "fill-in-the-gaps" for those unusual cases in which a particular channel of information was not collected.

The results of the analysis are subsequently presented; starting with some general statistics and followed by comparisons of the average fuel efficiencies observed from vehicles operating with different payload levels. A preliminary assessment of duty cycle variability is also presented for both the combination trucks and the transit buses.

\subsection{DATA AND ORGANIZATION}

The organization and accessibility of the information, as well as its accuracy, play a key role in the quality of any data analysis. Moreover, organization and accessibility of the data collected are particularly important when the size of the information database is significantly large. The field tests conducted as part of this project (more than 70 channels of data collected at $5 \mathrm{~Hz}$ for six vehicles) produced a database with a size of $190 \mathrm{~GB}$ of uncompressed information (see Table 4).

Table 4. Size and Number of Data Files Generated in the Project

\begin{tabular}{|c|c|c|c|c|c|c|c|c|c|}
\hline & \multicolumn{4}{|c|}{ MTDC Vehicle: Combination Trucks } & \multicolumn{4}{|c|}{ MTDC Vehicle: Transit Buses } & \multirow[b]{2}{*}{$\begin{array}{l}\text { Grand } \\
\text { Total }\end{array}$} \\
\hline & 1 & 2 & 3 & $\begin{array}{l}\text { Total } \\
\text { for } \\
\text { Vehicles } \\
1-3\end{array}$ & 4 & 5 & 6 & $\begin{array}{l}\text { Total } \\
\text { for } \\
\text { Vehicles } \\
4-6\end{array}$ & \\
\hline $\begin{array}{c}\text { Number of SoMat } \\
\text { Information Files (SIF) }\end{array}$ & 179 & 123 & 149 & 451 & 227 & 140 & 211 & 578 & 1,029 \\
\hline SIF Files Total Size [GB] & 3.79 & 3.39 & 3.57 & 10.75 & 12.99 & 6.23 & 12.30 & 31.51 & 42.26 \\
\hline $\begin{array}{l}\text { Number of American } \\
\text { Standard Code for } \\
\text { Information Interchange } \\
\text { (ASCII) Files }\end{array}$ & 179 & 121 & 149 & 449 & 227 & 137 & 214 & 578 & 1027 \\
\hline ASCII Files Total Size [GB] & 16.27 & 13.83 & 15.46 & 45.56 & 59.83 & 27.37 & 57.95 & 145.16 & 190.72 \\
\hline
\end{tabular}

Because of the size and complexity of the information collected, a significant effort was required to prepare the raw data for the analysis. As explained elsewhere in this report, the information was collected using a DAS that saved the information in its proprietary format (i.e., SIF files [SoMat Information Files]; SoMat was the manufacturer of the equipment used in the data collection task). These binary files were saved using a data structure format that optimizes the size and accessibility of the information. The information stored in this proprietary format was exported by means of software developed by ORNL to a 
flat text (i.e., ASCII, [American Standard Code for Information Interchange]) format that is readable by any user.

For the Part 1 MTDC FOT, ORNL developed additional software that allowed the retrieval of the information collected "over the air." The protocol that was used to save the information collected by the instrumented vehicles was as follows. Every night (8:00 PM to 12:00 AM for the combination trucks, and 1:00 AM to 5:00 AM for the transit buses) the ORNL data retrieval software contacted each vehicle and retrieved the SIF file with the information collected during that particular day. The day-long SIF file was then translated into an easily readable format as explained above, and the information was parsed into the 70+ channels that were collected and saved to files that stored one-day's worth of data collection data. These files were named with the calendar date in which the data was collected (e.g., 20091113, for information collected on November 13, 2009), plus a one-digit identifier that identified the truck (1 to 6) that gathered the information.

Simultaneous with the formatting of the information from binary to ASCII, the software developed by ORNL also checked the validity of the data provided by the 70+ channels. There were instances in which the on-board sensors did not provide information due to, for example, a malfunction of the sensor ("sensor invalid data"), and other cases where the DAS had problems storing the data ("eDAQ invalid data"). Each channel had a numeric code that identified these types of problems, and those codes were stored in the file for that particular channel and record. The data cleansing software read this information and generated statistics that were automatically e-mailed every morning by the software to the ORNL researchers in charge of the data collection effort. This information allowed the researchers to determine when a certain sensor was systematically failing or when the failures were just "glitches" that occurred sporadically. In the first case, troubleshooting, repair/replacement of the particular sensor was accomplished as soon as it was feasible.

Other errors such as out-of-range errors and errors attributed to the GPS were also identified and indications of their occurrence were added to the summary reports. Any record that had any kind of error for any channel was marked with a code that allowed for the identification of those errors, and this information was added to the corresponding calendar date file as a new field.

\subsubsection{Spatial Information}

The spatial location provided by the on-board GPS (i.e., latitude, longitude, and altitude collected every 0.2 seconds), permitted the ORNL-developed software to determine the road classification (e.g., freeway) and identification (e.g., I-40) on which the vehicle was traveling, if it was traveling inside or outside an urban area, as well as the state in which the truck was located.

The determination of the road on which the truck was traveling was not an easy task, especially since databases with this type of information are not readily available. In a normal geo-coding procedure, a given spatial feature (e.g., a segment of roadway) is represented by a series of points defined by latitude, longitude, and sometimes altitude; and the attributes of that spatial feature (e.g., the name of the roadway, its classification, number of lanes, etc.) that are associated to these points. This information, kept in proprietary databases, is used by Geographic Information System (GIS) software to draw these features on a map such that when a coordinate is entered (e.g., the location of a truck as determined by the latitude and longitude provided by the on-board GPS device), it could be displayed by placing a symbol of some sort on its actual location (e.g., the off-ramp from eastbound I-75 at Exit 374 in Knoxville, Tennessee). A reverse geo-coding procedure is the process of finding the attributes of a certain feature given the latitude and longitude information of a given point within that feature. That is, and for this project, it was necessary to find the classification and name of a given roadway once a point on, or very close to that roadway was known. These points were obtained from the on-board GPS devices as the vehicles traveled 
and therefore were known. Software was written to determine the name and classification of a given spatial feature (roadways in this case) when a latitude-longitude point was provided.

The information collected in this way was added to the database of channels, data errors, and spatial information (city and state) described above. At this point, the collected information database was archived and three copies were made which were stored on different ORNL data servers and at different locations to minimize the risk of data loss.

\subsubsection{Vehicle Weight Information}

In any study involving the fuel efficiency of a vehicle, weight plays a key role. It was therefore paramount that this type of information be collected in this study. To do so, a device (Air-Weigh) which is described elsewhere in this report, was mounted on the three participating tractors and the three transit buses, and provided instantaneous weight measurements at the steer and drive axels ${ }^{7}$, which were saved as two of the 70+ data channels of the DAS. The Air-Weigh device was not mounted on any of the H.T. Hackney trailers. Based on vehicle weight data collected at commercial scales (i.e., Inspection Stations in Knox County, Tennessee), ORNL developed a model that predicted the trailer weight based on the tractor weight provided by the Air-Weigh device. This model, which is described in detail later in this report, was used to assign trailer weight to each one of the records in the database of data collected in this project. For the transit buses, the deployed Air-Weigh devices provided weight information for the entire vehicle, and therefore it was not necessary to develop a payload estimation model in this case.

\subsubsection{Additional Information}

Additional information relevant to the data analysis task was also generated and added to the database of data collected in this project. This included roadway slope (in percent), which was computed using the altitude data gathered with the help of the GPS device, and filtered to smooth out local fluctuations due to GPS errors; and filtered vehicle speed which was later used to determine acceleration, deceleration and constant speed intervals ${ }^{8}$. The filter used was a sliding exponential smoothing filter that considered the median of eleven observations before, and eleven observations after the current point.

\subsubsection{Information Indexing}

In order to efficiently find and retrieve specific information from the large database of information collected in this project (i.e., $190 \mathrm{~GB}$ ) it was necessary to index the information and to create a database of pointers that would allow for the quick retrieval of data records that comply with possibly multiple search criteria specified by the analyst.

Several aspects of the collected information were expected to play a relevant role in the data analysis; for example, the following situations were explored.

- a vehicle moving on a freeway vs. a vehicle moving on a surface street

- a vehicle moving in an urban area vs. a vehicle moving in a rural area

- a vehicle moving under rainy conditions vs. a vehicle moving under clear weather

- a vehicle moving during the morning peak hour vs. a vehicle moving during mid-morning offpeak hours

- a vehicle moving vs. a vehicle in a stationary position; and many others

\footnotetext{
${ }^{7}$ The Air-Weigh device uses inputs from the vehicle air-suspension system. For the tractor, only the drive axle weight is measured; the steer axle weight is computed by the Air-Weigh device.

${ }^{8}$ Filtering was needed because of the inherent fluctuations in the data, which if not filtered would have introduced errors into the data analyses.
} 
For these situations, the ORNL-developed software identified, within the daily data files previously generated, the records in which each attribute is valid, and where it is not valid. This indexing allowed for a very efficient management of the information and was used in the data analysis task to allow for the selection of files that contained only the specific information of interest; for example, a vehicle traveling in urban areas, during the morning peak-hour, during rainy weather.

A search engine, that uses the index pointers was developed and included with the DCGenT Prototype to help with the identification and retrieval of information that satisfies one or more user-selected criteria simultaneously. This newly collected information was added to the already existing HTDC database made available to the user through a menu choice that allows selecting information about Class- 8 trucks, mid-size combination trucks, or transit buses (see Figure 13). Figure 14 and Figure 15 show screen captures of the data selection criteria that are part of the DCGenT Prototype for the combination trucks and transit buses, respectively. Those criteria include the following:

1) time of day (peak and off-peak periods),

2) precipitation (indicated by wiper status),

3) roadway type (freeways or surface streets),

4) spatial location (urban or rural areas),

5) congestion level based on vehicle speed and road type,

6) topography (different type of terrains from upslope to flat to down slope),

7) specific vehicle (one or more of the three instrumented tractors, or one or more of the three instrumented buses),

8) vehicle dynamic condition (e.g., truck stationary, truck accelerating, etc.), and

9) vehicle weight (in four levels: empty, light load, medium load, and heavy load).

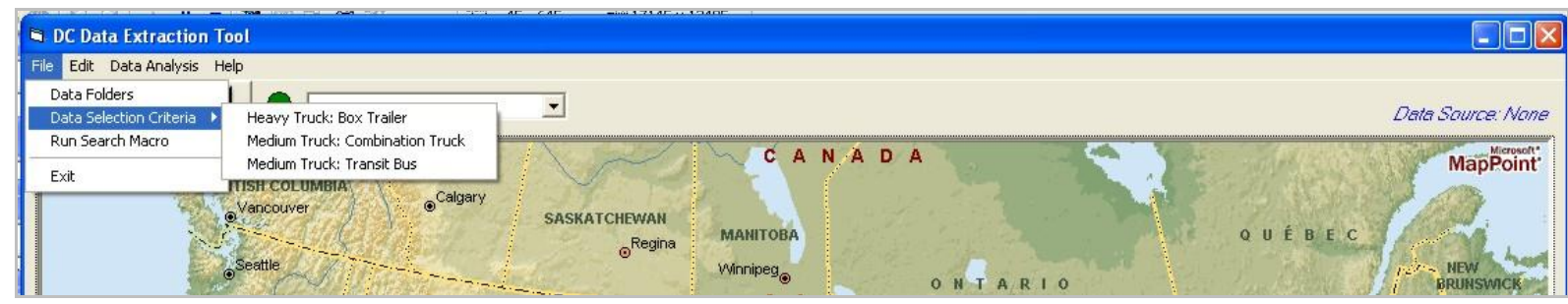

Figure 13. Vehicle Class and Vocation Selection Menu 


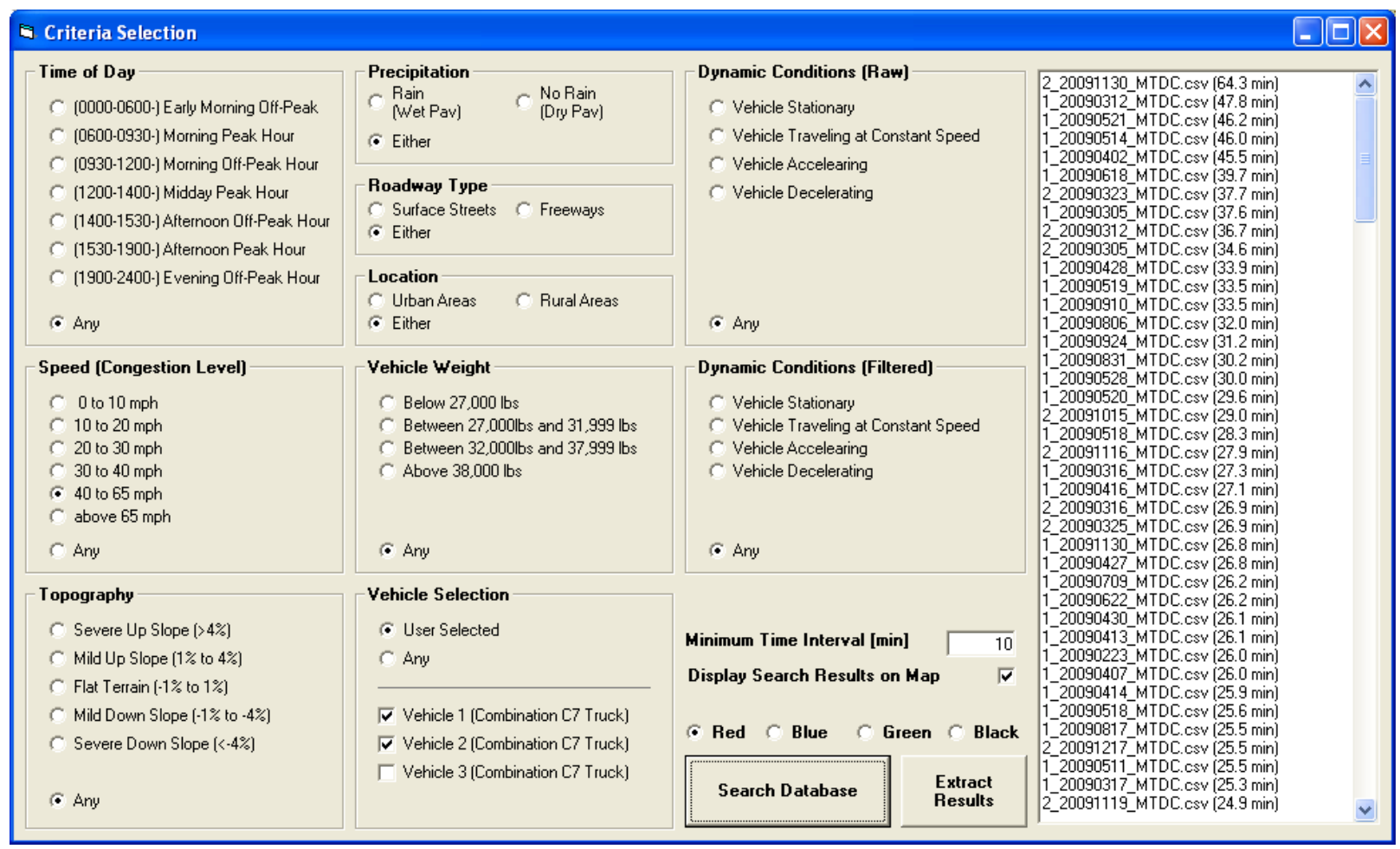

Figure 14. Criteria Selection - Class-7 Combination Trucks

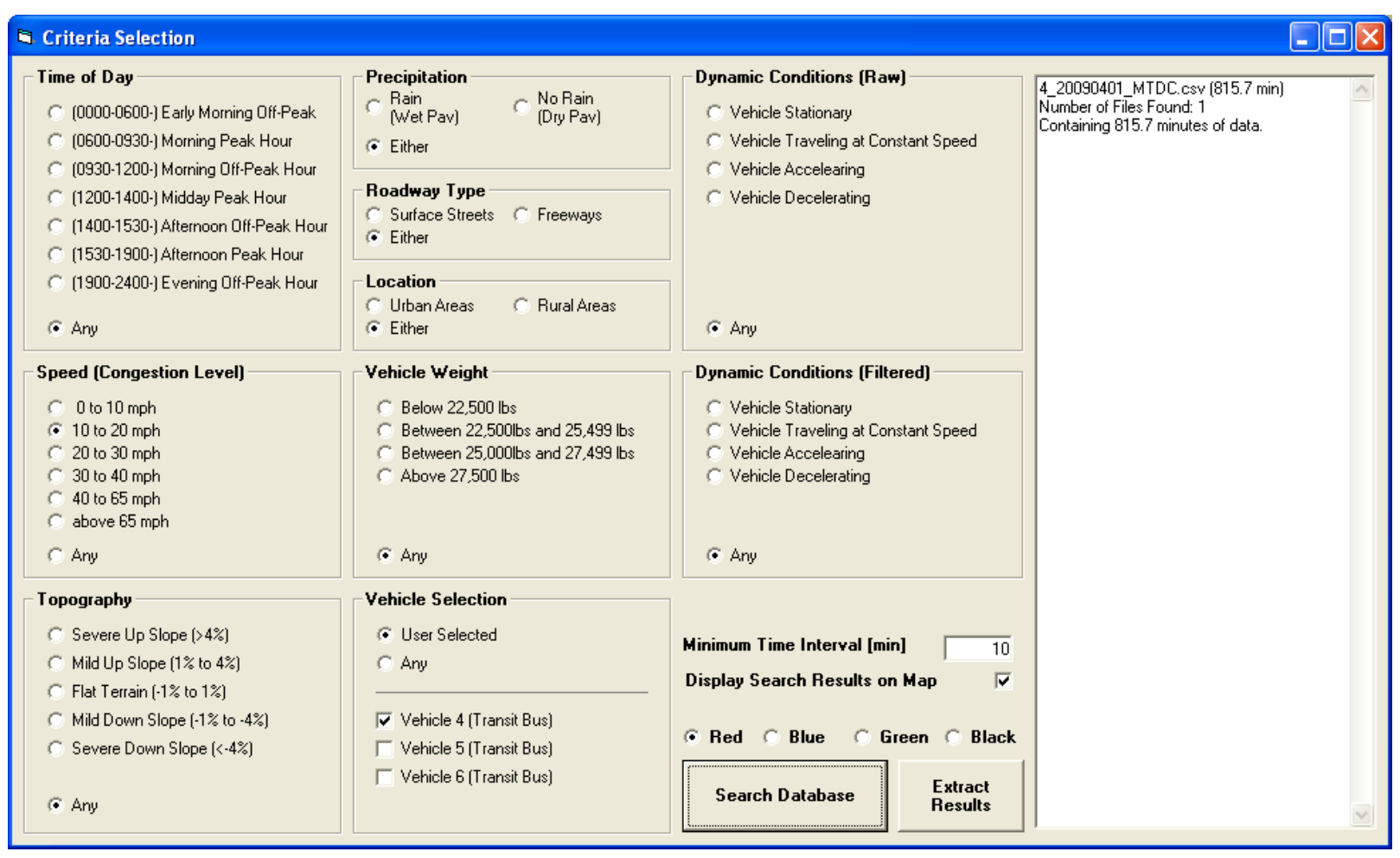

Figure 15. Criteria Selection - Class-7 Transit Buses 
After the user has selected all of the searching criteria of interest and the Search Database button is pressed, the software finds the list of pointers for the chosen criteria and retrieves from the database all of the records that satisfy these criteria simultaneously.

\subsection{ADDITIONAL DEVELOPED MODELS AND DATA CALIBRATION}

\subsubsection{Vehicle Weight Model}

As discussed briefly in the last section, none of the participating trailers were instrumented with AirWeigh devices, and therefore the truck's total weigh information could not be directly assessed. A truck total-weight-prediction model was developed that used the tractor weight, which was always available, total vehicle weight information obtained at certified commercial vehicle scales, and the physics related to inertia. That is, as a truck accelerates, the weight of the trailer would resist the change in speed by inducing a force proportional to that weight. This force would impede the movement of the tractor and would appear as a loss of weight as registered by the Air-Weigh device on the tractor, which determines axle weight by sampling the pressure of the air suspension system. Therefore, by observing the behavior (i.e., the weight readings provided by the Air-Weigh device) of the instrumented tractor under different acceleration levels and for known vehicle weights (as obtained from commercial vehicle scales), it would be possible, in theory, to characterize the tractor and trailer's response for a particular payload. A parametric study of payload weights would theoretically provide a truck response map that can be used to estimate the payload weight of tractors mated to un-instrumented trailers.

This theory was tested using vehicle dynamics and tractor weight information collected for the cases in the database in which the vehicle total weight was known from external sources. The tractor weight was sampled within pre-specified acceleration intervals, ranging from -1.4 to $1.4 \mathrm{~m} / \mathrm{s}^{2}$, and linear regression models ${ }^{9}$ were developed to fit the "loss of weight" observed as a function of the vehicle acceleration. Figure 16 and Figure 17 show a representation of the cabin weight as a function of vehicle acceleration for a loaded truck (i.e., a truck leaving the warehouse at the beginning of the day) and an empty truck (a truck returning to the warehouse after delivering the entire payload), respectively.

9 A linear fitting function was used since the force opposing the movement of the tractor is proportional to the acceleration (and trailer weight). 


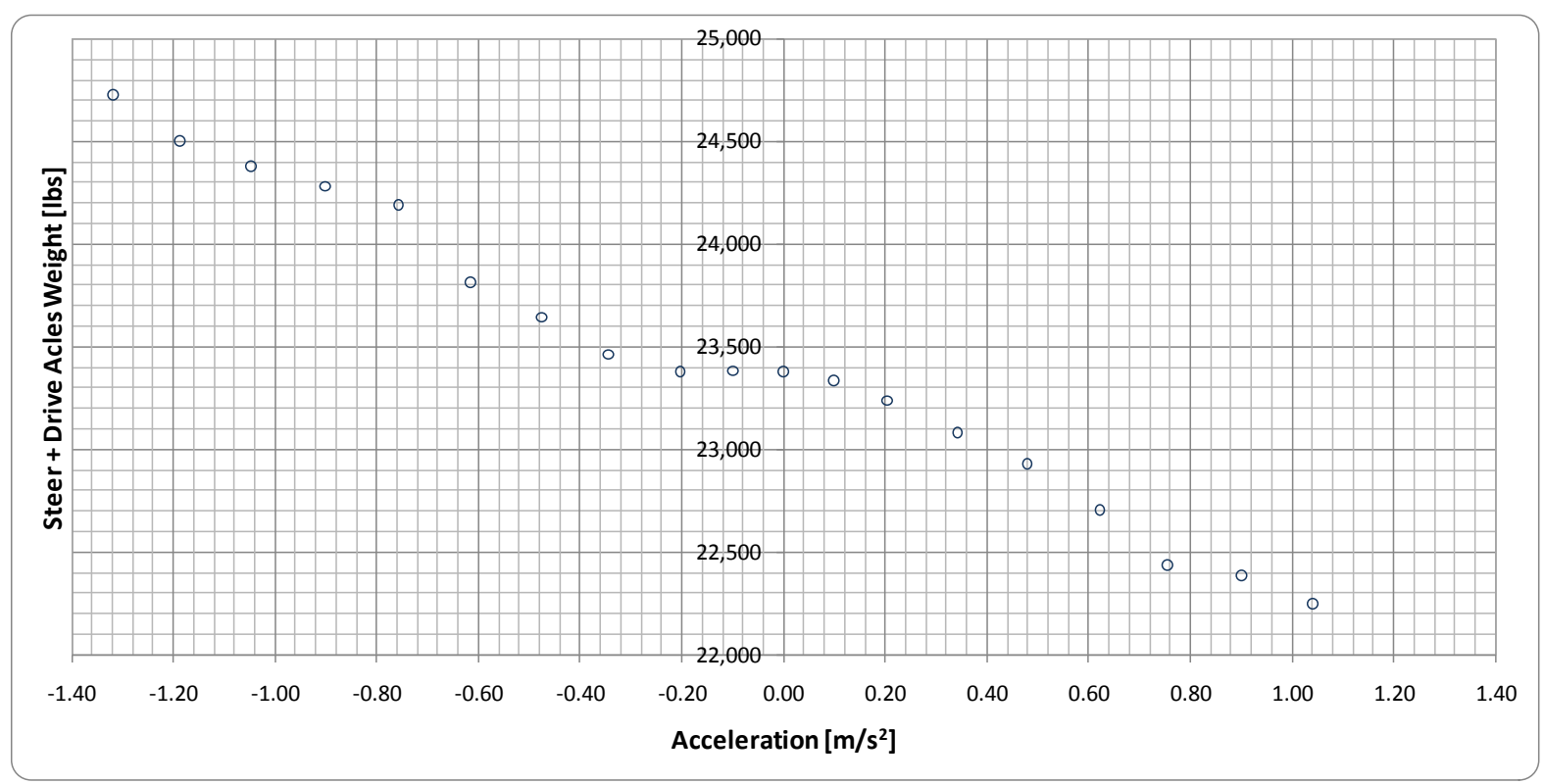

Figure 16. Tractor Weight vs. Acceleration - Loaded Truck

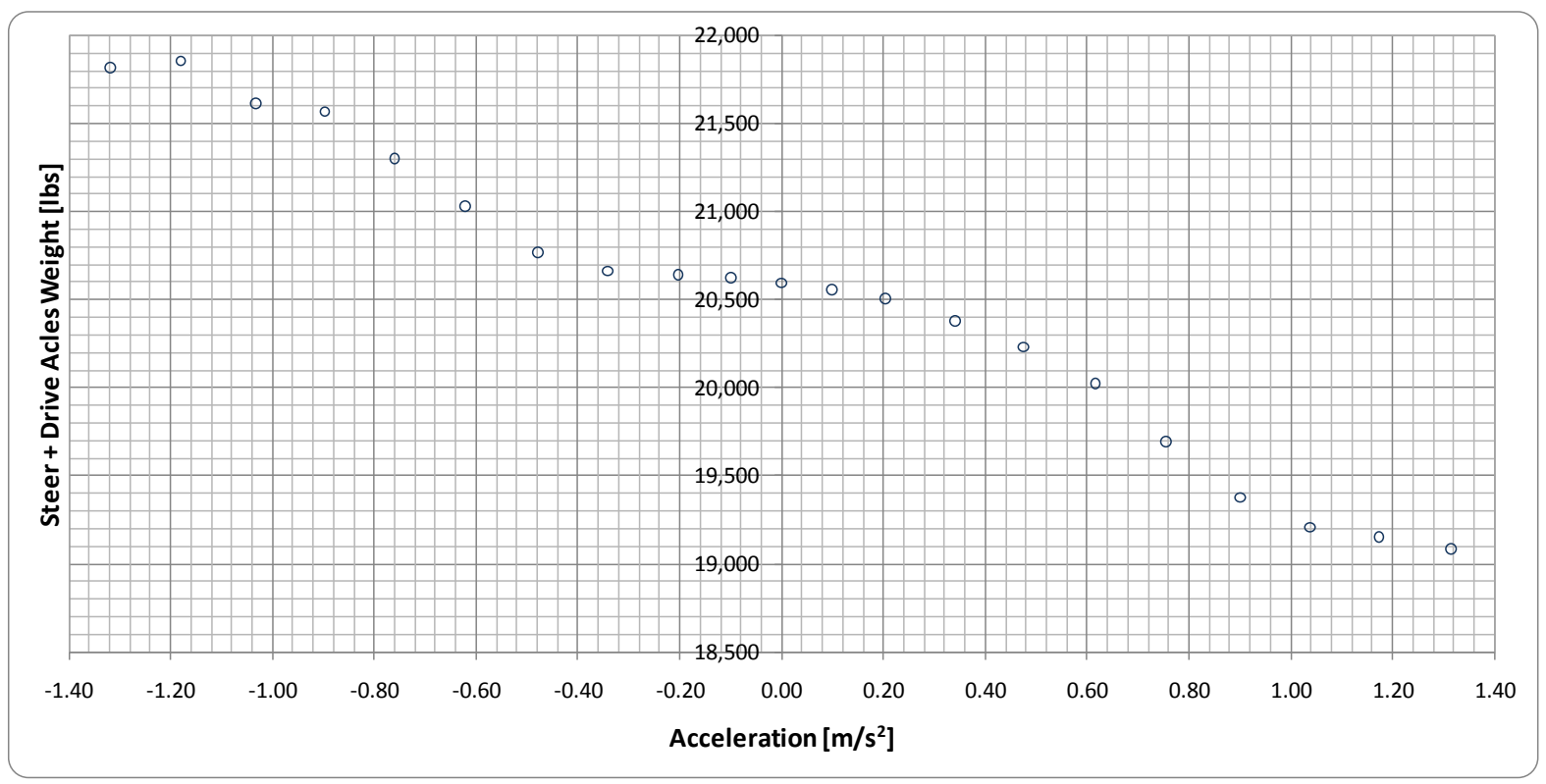

Figure 17. Tractor Weight vs. Acceleration - Empty Truck

Notice that in both cases the shape of the tractor weight vs. acceleration relationship follows the same pattern, with three distinct regions. Region 1: a close-to-linear region for the vehicle decelerating (braking) in which the Air-Weigh device registers less weight as the vehicle acceleration increases (i.e., the deceleration becomes smaller in absolute value). Region 2: an almost "flat" region with acceleration values ranging from -0.20 to $0.15 \mathrm{~m} / \mathrm{s}^{2}$ (note: when the vehicle acceleration is very low in absolute value, then there is no "shifting" in weight among the vehicle axles and therefore the weight-acceleration relationship is constant). Region 3: another close-to-linear region for the vehicle accelerating in which the Air-Weigh device registers less weight as the vehicle acceleration increases. 
In both cases (i.e., loaded and empty trucks) Regions 1 and 3 are very similar. Therefore, and in order to be consistent with the HTDC weight prediction models, the acceleration region (Region 3) was used to develop the total-truck weight prediction model (see Capps et al., 2008, ORNL/TM-2008/122). Figure 18 and Figure 19 show the linear regression lines as well as the parameters of these lines, and the $\mathrm{R}^{2}$ (or "goodness of fit" parameter) for Region 3 of the two payload cases. The high $\mathrm{R}^{2}$ indicates that, as was expected, the observed weight of the tractor decreases in a way that is very close to a linear function as the vehicle acceleration increases. Notice also that, as expected, the intercept of the linear model (i.e., weight of tractor when the vehicle is stationary) is lower for the empty-truck case than for the loaded one.

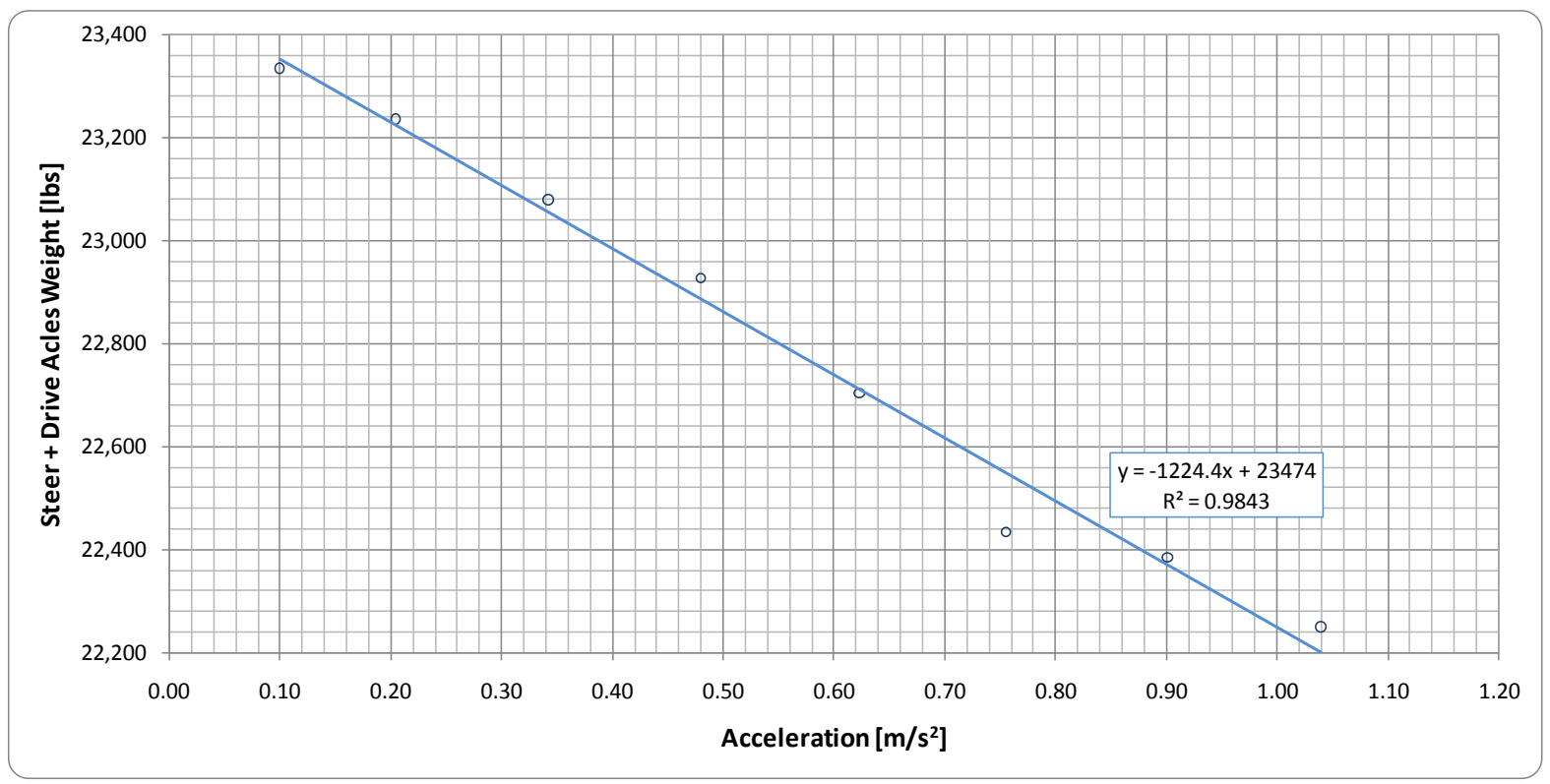

Figure 18. Linear Model for Tractor Weight vs. Acceleration - Loaded Truck

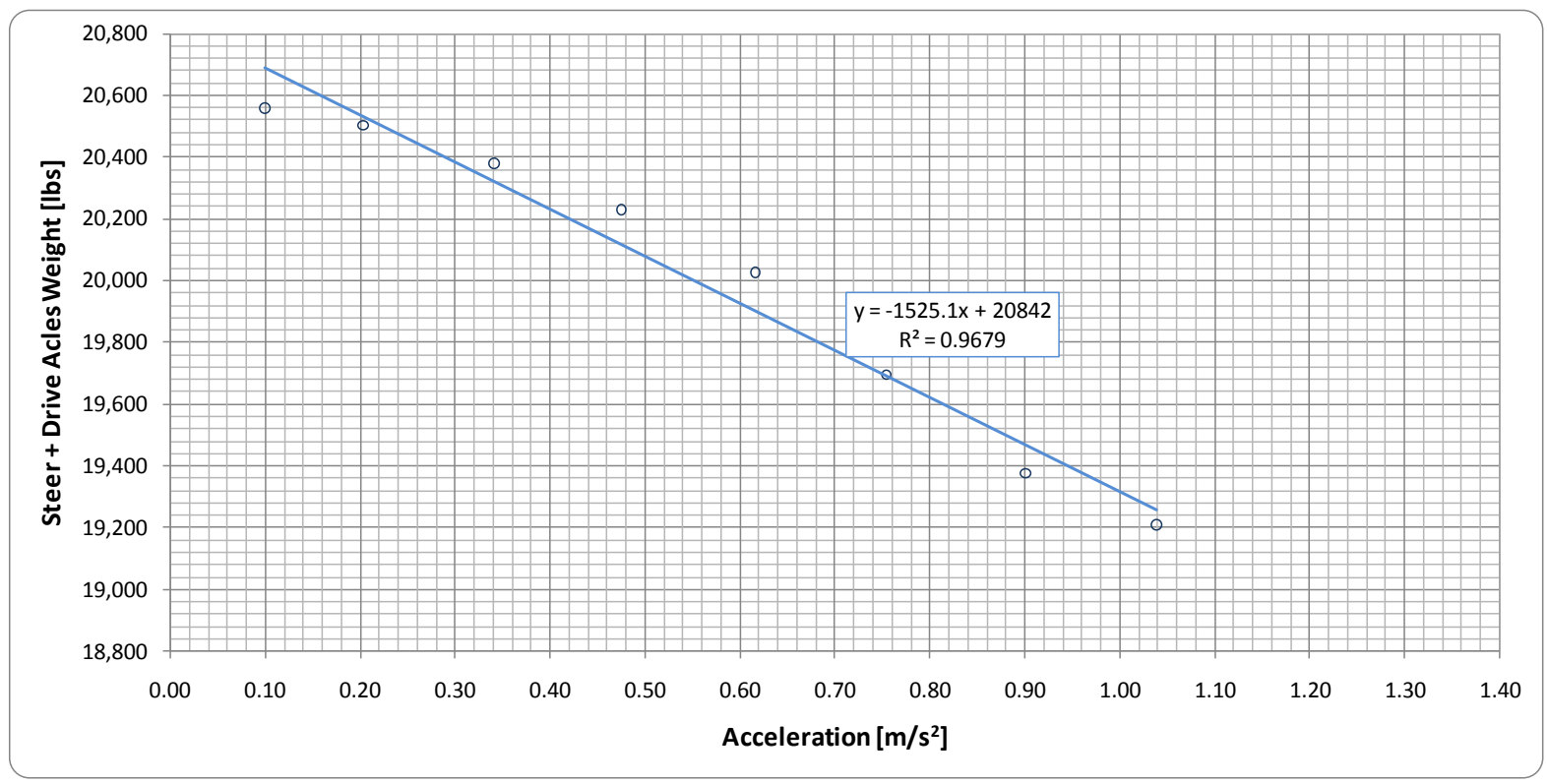

Figure 19. Linear Model for Tractor Weight vs. Acceleration - Empty Truck 
In order to develop a truck weight prediction model, the information (i.e., vehicle speed and tractor weight obtained from the on-board Air-Weigh device) collected from trips in which the vehicle total weight was obtained from commercial vehicle scales was used to generate the linear regression parameters of the tractor weight $t w$ (i.e., its slope $m$ and intercept $b$ ) as a function of vehicle acceleration $v a$ (call this linear regression model, M1). At the same time, the total truck weight $t t w$ was noted; this parameter was obtained from exogenous sources (commercial vehicle scales). All of these three parameters $(m, b$, and $t t w)$ were determined using information from several trips in which the drivers of the vehicles stopped at an Inspection Station in Knox County, Tennessee at the beginning of their shift (loaded truck) and at the end of the working day (empty truck). The vehicle speed and Air-Weigh information collected from the on-board sensors while the truck traveled from the warehouse to the Inspection Station and from there to their first stop (i.e., two trips in which the vehicle total weight was constant, except for the fuel consumed) was used to determine parameters $m$ and $b$ for a loaded vehicle. The other parameter, $t t w$, was obtained from the weight ticket issued by the Tennessee Highway Patrol at the Inspection Station. Similarly, on certain days the drivers stopped at the Knox County Inspection Station at the end of the working day. Information collected during the trips from the last delivery stop on that day to the Inspection Station and from the latter to the warehouse was used to determine parameters $m$ and $b$ for an empty vehicle. The other parameter, $t t w$, was obtained from the weight ticket issued at the Inspection Station.

A second linear regression model (M2) was developed using the observed $m$ 's and $b$ 's, as independent variables, and the $t t w$ 's as the dependent variable. M2 models were developed for each one of the three tractors. The external information collected at the Knox County Inspection Station to develop these M2 models (i.e., steer and drive axle weights, trailer axle weight, and total vehicle weight $t t w s$ ) is presented in Table 5 and Table 6 for the loaded and empty vehicle cases, respectively.

Table 5. Weight Ticket Information - Loaded Truck

\begin{tabular}{|c|c|c|c|c|c|c|}
\hline \multirow{2}{*}{ Date } & \multirow{2}{*}{ Time } & \multirow{2}{*}{$\begin{array}{c}\text { Vehicle } \\
\text { ID }\end{array}$} & \multicolumn{4}{|c|}{ Axle Weight [lbs] } \\
\hline & & & Steer & Drive & Trailer & Total \\
\hline $9 / 28 / 2009$ & 06:04 & 1 & 9,460 & 14,400 & 9,960 & 33,820 \\
\hline $9 / 28 / 2009$ & 06:01 & 3 & 9,360 & 13,080 & 9,820 & 32,260 \\
\hline $9 / 30 / 2009$ & 06:00 & 1 & 9,700 & 14,460 & 9,620 & 33,780 \\
\hline $9 / 30 / 2009$ & $05: 52$ & 2 & 9,560 & 14,720 & 10,200 & 34,480 \\
\hline $9 / 30 / 2009$ & $06: 33$ & 3 & 9,040 & 13,800 & 9,500 & 32,340 \\
\hline $10 / 5 / 2009$ & 06:02 & 1 & 9,460 & 15,740 & 11,860 & 37,060 \\
\hline $10 / 5 / 2009$ & 05:11 & 2 & 9,460 & 14,000 & 10,200 & 33,660 \\
\hline $10 / 5 / 2009$ & $06: 40$ & 3 & 9,340 & 13,440 & 8,820 & 31,600 \\
\hline $3 / 15 / 2010$ & 08:56 & 1 & 9,940 & 14,660 & 9,520 & 34,120 \\
\hline $3 / 15 / 2010$ & $05: 12$ & 2 & 9,080 & 14,400 & 11,960 & 35,440 \\
\hline $3 / 15 / 2010$ & 05:55 & 3 & 9,100 & 13,100 & 8,540 & 30,740 \\
\hline $3 / 16 / 2010$ & $07: 47$ & 1 & 9,860 & 15,020 & 13,240 & 38,120 \\
\hline $3 / 16 / 2010$ & 07:14 & 2 & 9,060 & 13,620 & 11,160 & 33,840 \\
\hline $3 / 16 / 2010$ & $04: 28$ & 3 & 9,280 & 13,720 & 11,260 & 34,260 \\
\hline $3 / 18 / 2010$ & 05:09 & 1 & 9,720 & 14,560 & 11,720 & 36,000 \\
\hline $3 / 18 / 2010$ & 04:06 & 2 & 9,260 & 14,600 & 10,540 & 34,400 \\
\hline $3 / 18 / 2010$ & 06:10 & 3 & 9,180 & 13,400 & 9,540 & 32,120 \\
\hline
\end{tabular}


Table 6. Weight Ticket Information - Empty Truck

\begin{tabular}{|c|c|c|c|c|r|c|}
\hline \multirow{2}{*}{ Date } & \multirow{2}{*}{ Time } & \multirow{2}{*}{$\begin{array}{c}\text { Vehicle } \\
\text { ID }\end{array}$} & \multicolumn{4}{|c|}{ Axle Weight [lbs] } \\
\cline { 5 - 7 } & & Steer & Drive & Trailer & Total \\
\hline $10 / 15 / 2009$ & $16: 18$ & 1 & 9,620 & 11,820 & 7,480 & 28,920 \\
$10 / 15 / 2009$ & $16: 39$ & 2 & 9,080 & 10,960 & 6,960 & 27,000 \\
$10 / 15 / 2009$ & $14: 11$ & 3 & 9,020 & 10,920 & 6,740 & 26,680 \\
\hline $10 / 16 / 2009$ & $14: 43$ & 1 & 9,480 & 11,820 & 7,260 & 28,560 \\
$10 / 16 / 2009$ & $12: 42$ & 2 & 9,020 & 11,200 & 7,380 & 27,600 \\
$10 / 16 / 2009$ & $15: 03$ & 3 & 9,240 & 11,000 & 6,860 & 27,100 \\
\hline $3 / 15 / 2010$ & $13: 15$ & 2 & 8,860 & 11,140 & 6,920 & 26,920 \\
$3 / 15 / 2010$ & $14: 09$ & 3 & 8,880 & 10,840 & 6,740 & 26,460 \\
\hline $3 / 16 / 2010$ & $16: 27$ & 1 & 9,480 & 11,740 & 7,400 & 28,620 \\
$3 / 16 / 2010$ & $15: 36$ & 2 & 8,900 & 10,760 & 6,820 & 26,480 \\
$3 / 16 / 2010$ & $13: 29$ & 3 & 9,520 & 11,000 & 6,960 & 27,480 \\
\hline $3 / 18 / 2010$ & $15: 01$ & 1 & 9,380 & 11,600 & 7,480 & 28,460 \\
$3 / 18 / 2010$ & $14: 02$ & 2 & 9,000 & 11,020 & 6,860 & 26,880 \\
$3 / 18 / 2010$ & $13: 47$ & 3 & 9,140 & 10,860 & 6,880 & 26,880 \\
\hline
\end{tabular}

The methodology to determine truck total weight was as follows. Using the tractor Air-Weigh readings parameters $m, b$, and $t w$ (i.e., model M1 parameters) were generated for each time segment in those trips in which the truck was moving, as explained above. Those parameters were then used as inputs in the corresponding tractor M2 model to predict the truck total weight. The latter quantity was then assigned to the corresponding records in the database of collected information.

\subsubsection{Calibration of Data Bus Fuel Readings}

Due to the focus of the MTDC project on the study of fuel efficiency, the available fuel-related vehicle data bus signals were studied to understand the accuracy of these signals. Prior to initiation of the FOT, a short-term fuel study was performed to understand the relationship between the actual fuel usage and similar data available from the vehicle's data bus. Drivers of four combination vehicles from the partnering fleet kept a fuel log for the tractor units for one month. ${ }^{10}$ Records from the vehicles' electronic on-board recorders (EOBRs) provided similar information based on signals from the J1708 vehicle data bus. Of the 20 segments for which manual and automatically-recorded (J1708-based) logs were available, there was one outlier with a value of more than four standard deviations below the mean. The remaining data is shown in Figure 20.

10 The refrigerated trailer units are fueled separately and the amount of this fuel is not included in the analysis. 


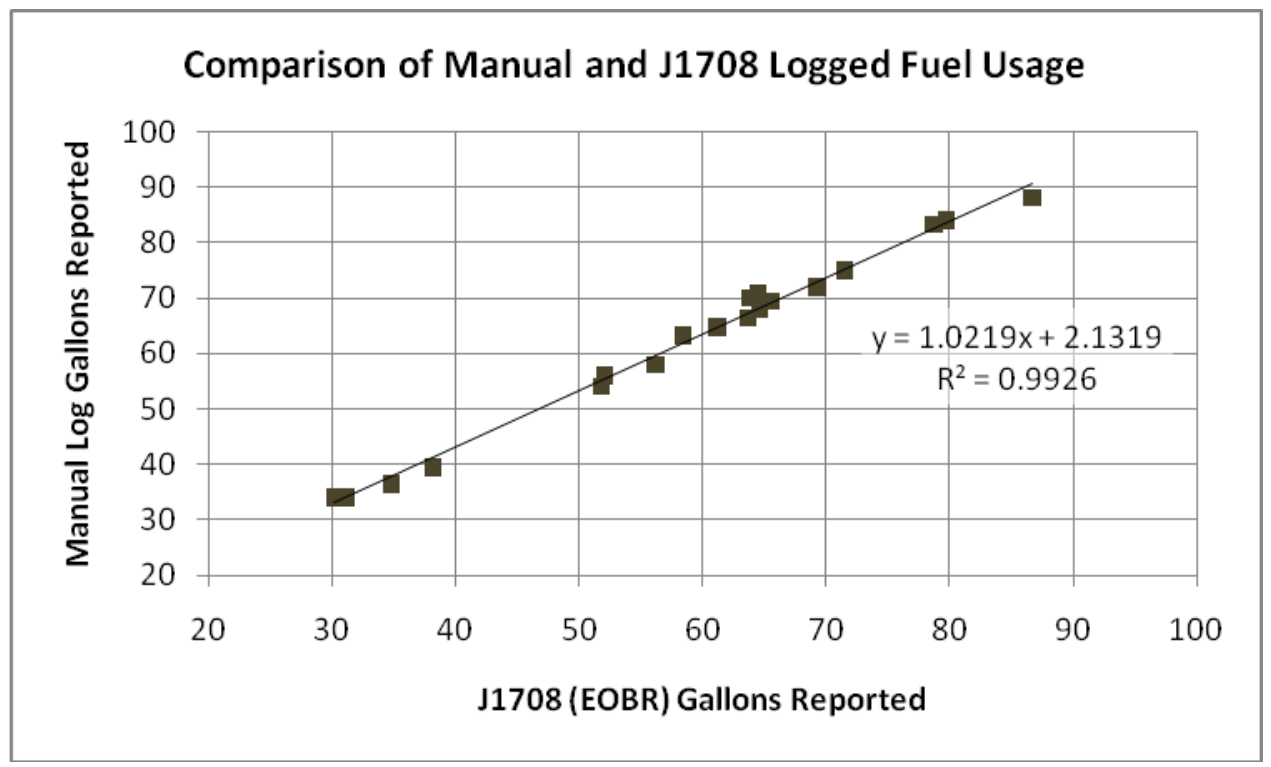

Figure 20. Gallons of Fuel Used - Manual Log Values vs. EOBR-reported Values

This plot indicates that the reported gallons of fuel used from the vehicle's J1708 data bus provides an accurate representation of the actual fuel used. However, the DAS used in the FOT recorded this value from the newer J1939 data bus rather than the older J1708 data bus. EOBR logs were obtained for one month of recorded duty cycle data to compare these two data buses. As shown in Figure 21, the two data sets were virtually identical, indicating that the J1939 provides a basis for accurate fuel usage calculations.

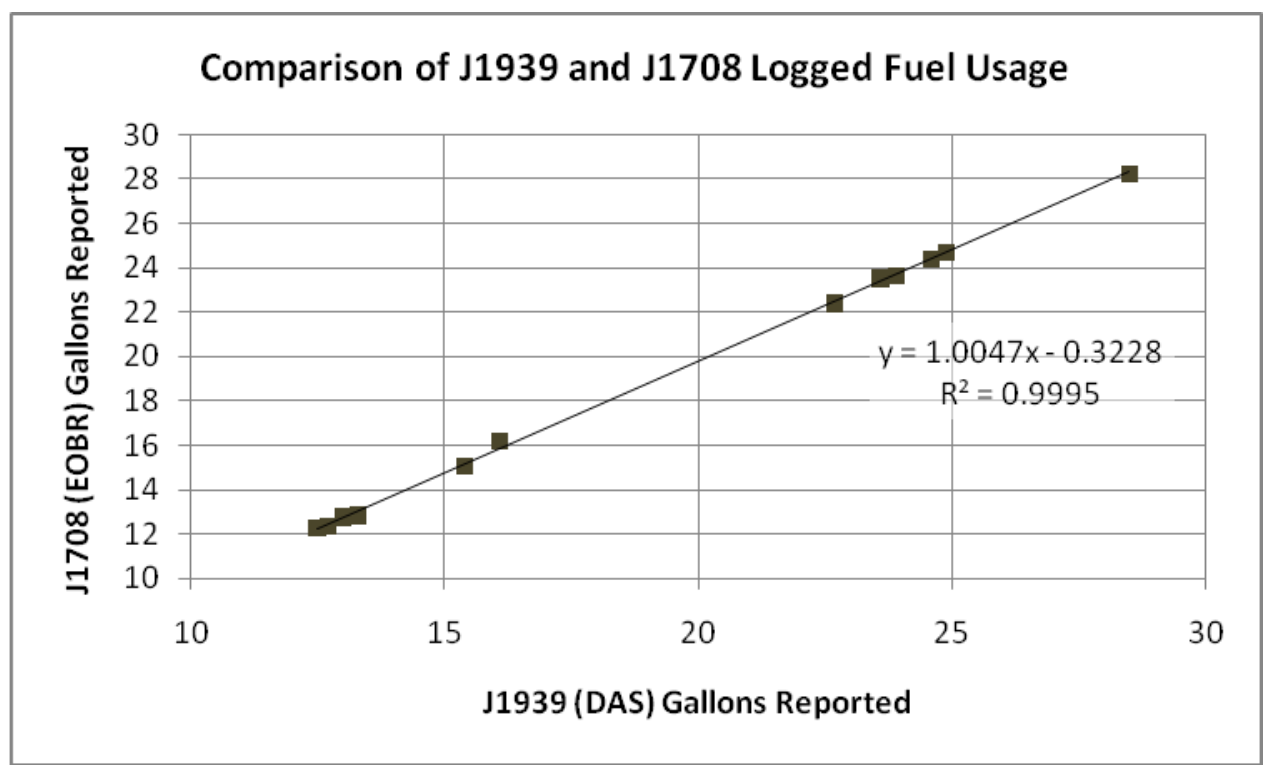

Figure 21. Gallons of Fuel Used - EOBR-Reported Values vs. DAS-recorded Values

\subsection{GENERAL STATISTICS}

The main focus of the data analysis was on the fuel efficiency of Class-7 vehicles operating in different vocations and under different payload conditions. The analysis presents some general statistics that summarize the operations of the two vocations (i.e., combination trucks and transit buses) that 
participated in the first phase of the MTDC project. An analysis of the fuel efficiency of these vehicles follows, with the payload dimension added later in this chapter. To add this dimension, a vehicle total weight was developed for the combination trucks as discussed above, and it was used to assign total vehicle weight to each record in the database. An investigation of the variability of duty cycles (both on freeways and on surface street) is also presented in this section.

\subsubsection{General Statistics}

During the one-year data collection period, the six participating vehicles logged over 95,000 miles (45,400 for the combination trucks and 49,400 for the transit buses) and consumed over 17,000 gallons of fuel $(6,000$ for the combination trucks and 11,300 for the transit buses), while conducting business in the East Tennessee area. Figure 22 to Figure 24 show the area of operations of the three combination trucks while Figure 25 to Figure 27 present the superimposed routes for the three transit buses during the oneyear data collection period.

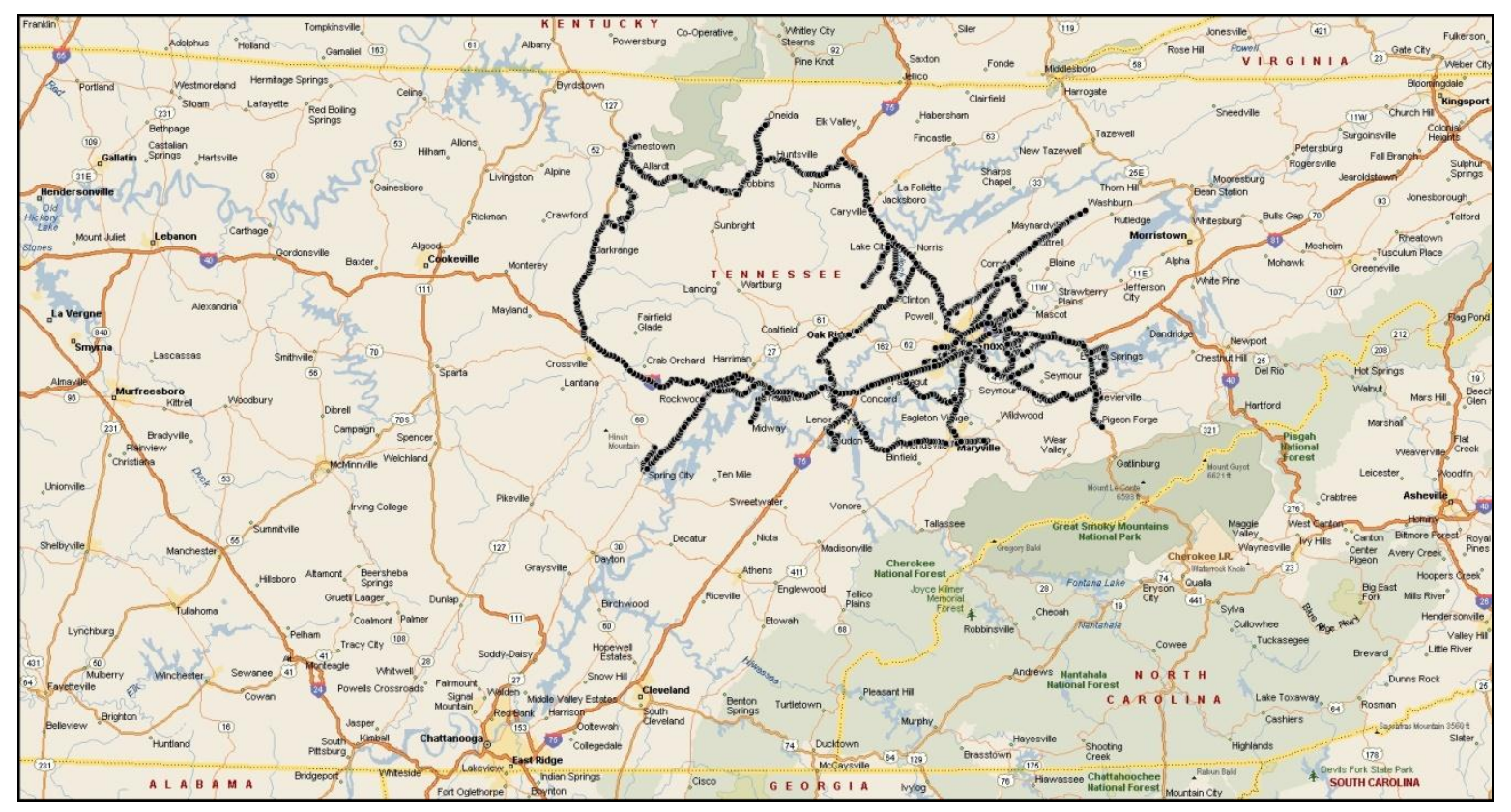

Figure 22. Combination Truck 1 (Vehicle 1) Coverage Area 


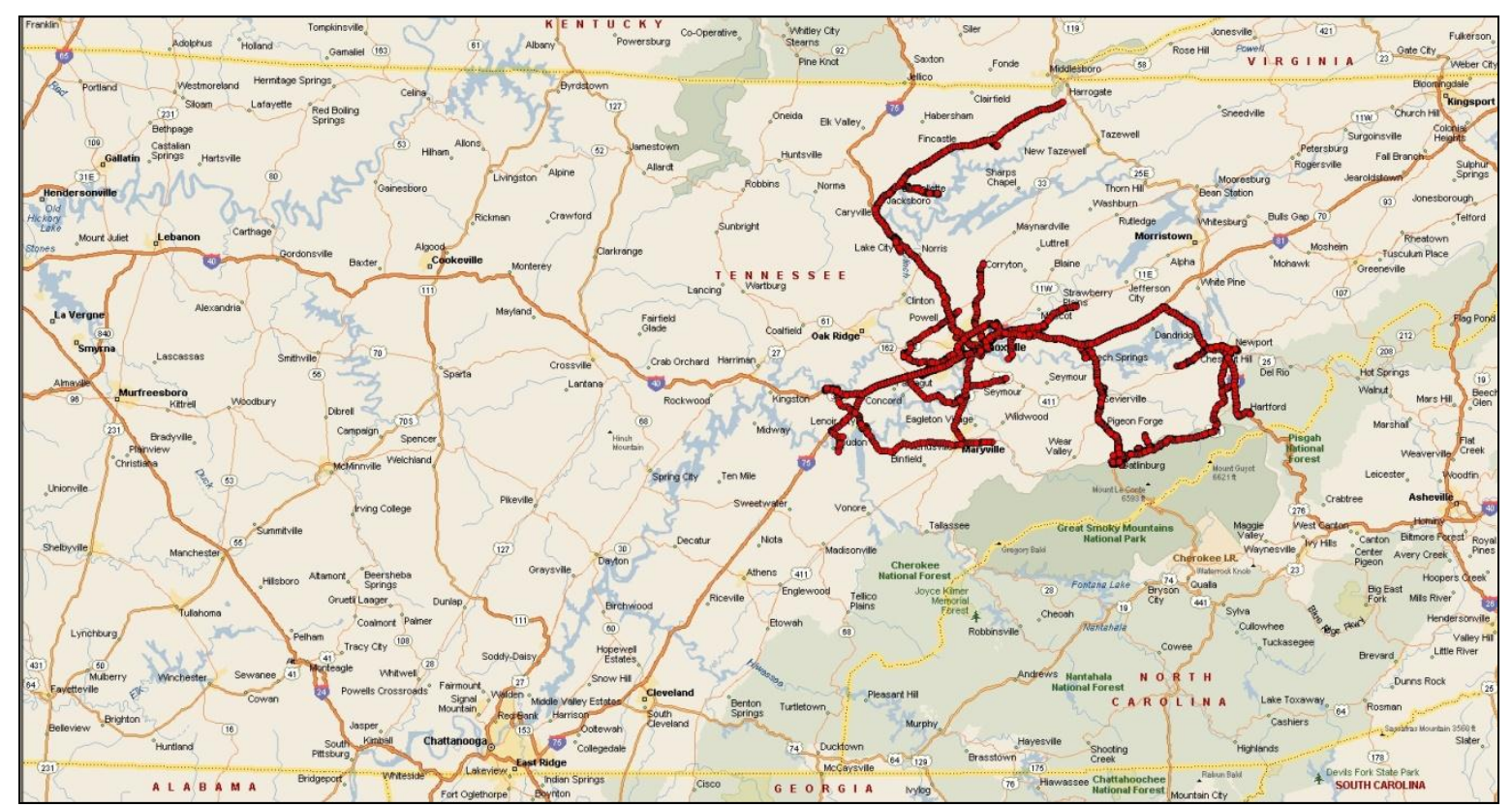

Figure 23. Combination Truck 2 (Vehicle 2) Coverage Area

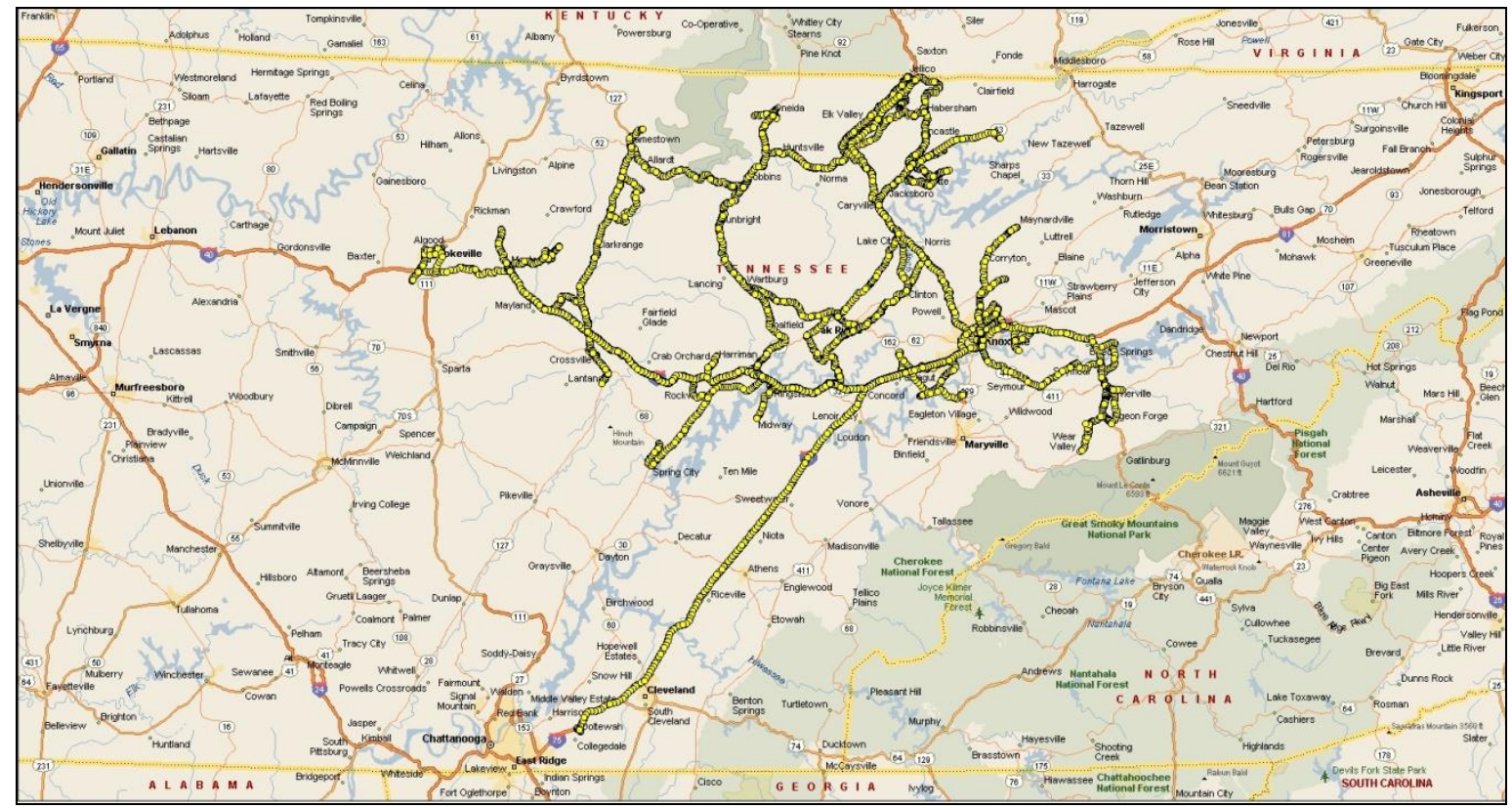

Figure 24. Combination Truck 3 (Vehicle 3) Coverage Area 


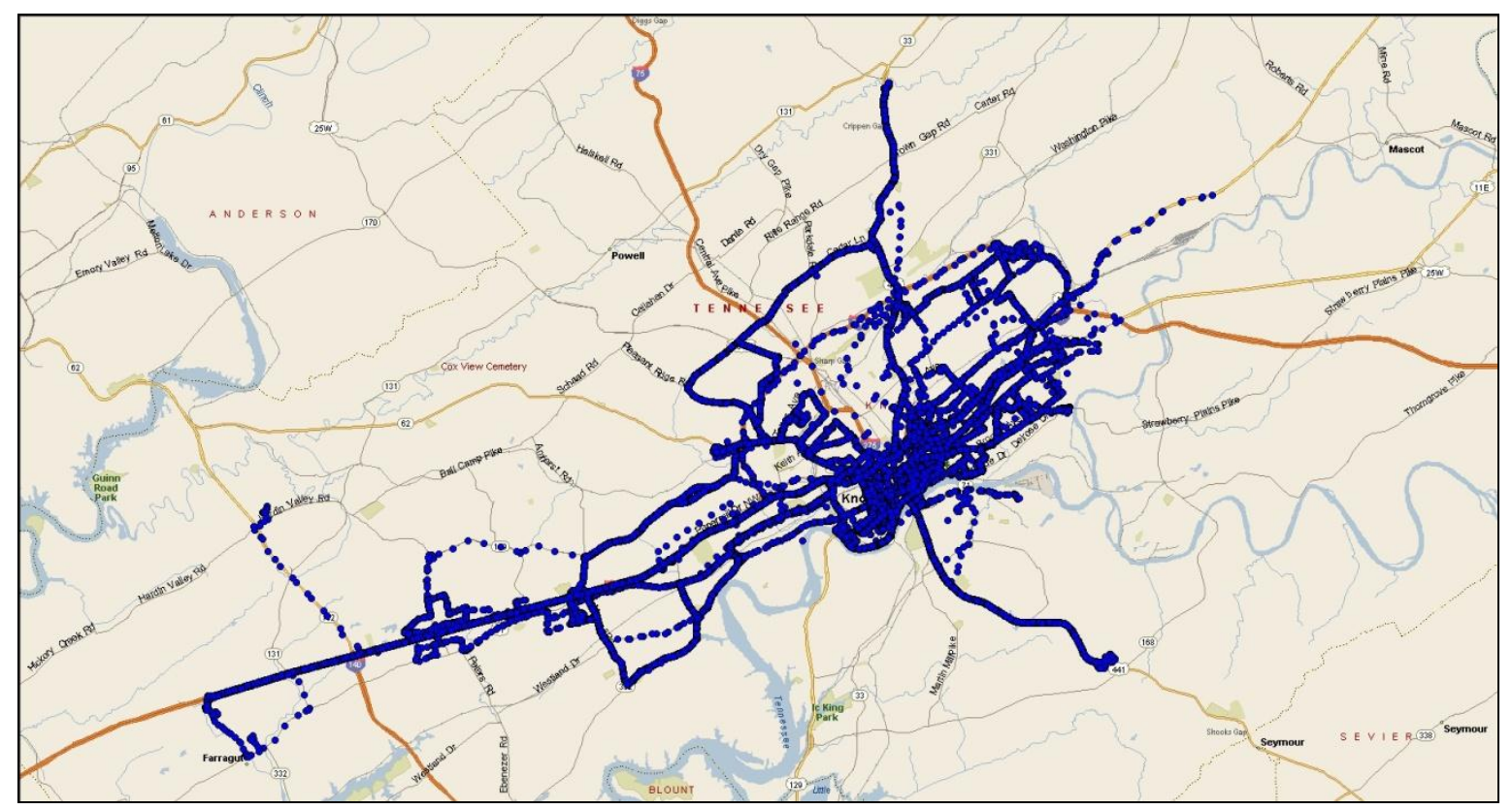

Figure 25. Transit Bus 1 (Vehicle 4) Coverage Area

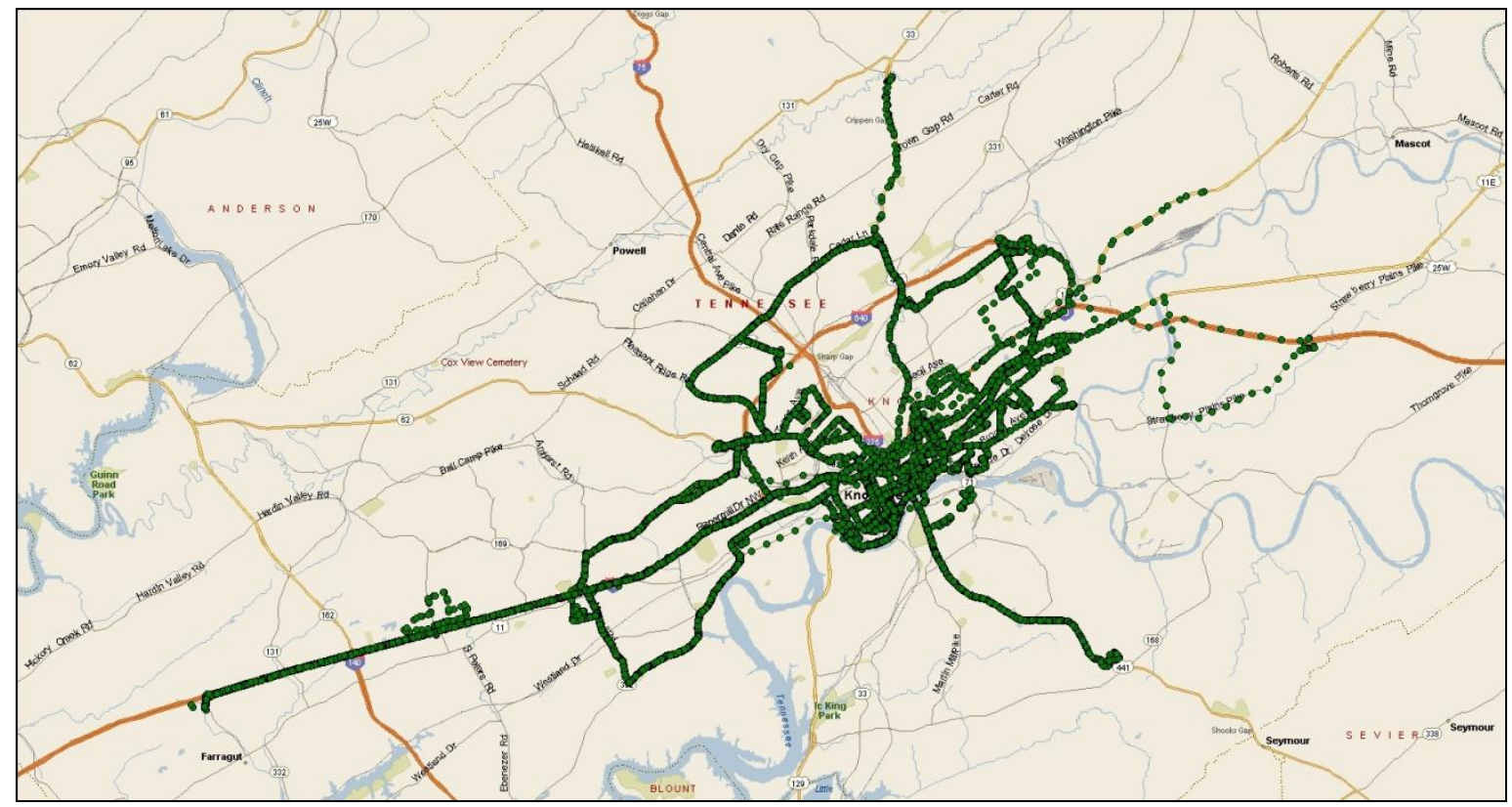

Figure 26. Transit Bus 2 (Vehicle 5) Coverage Area 


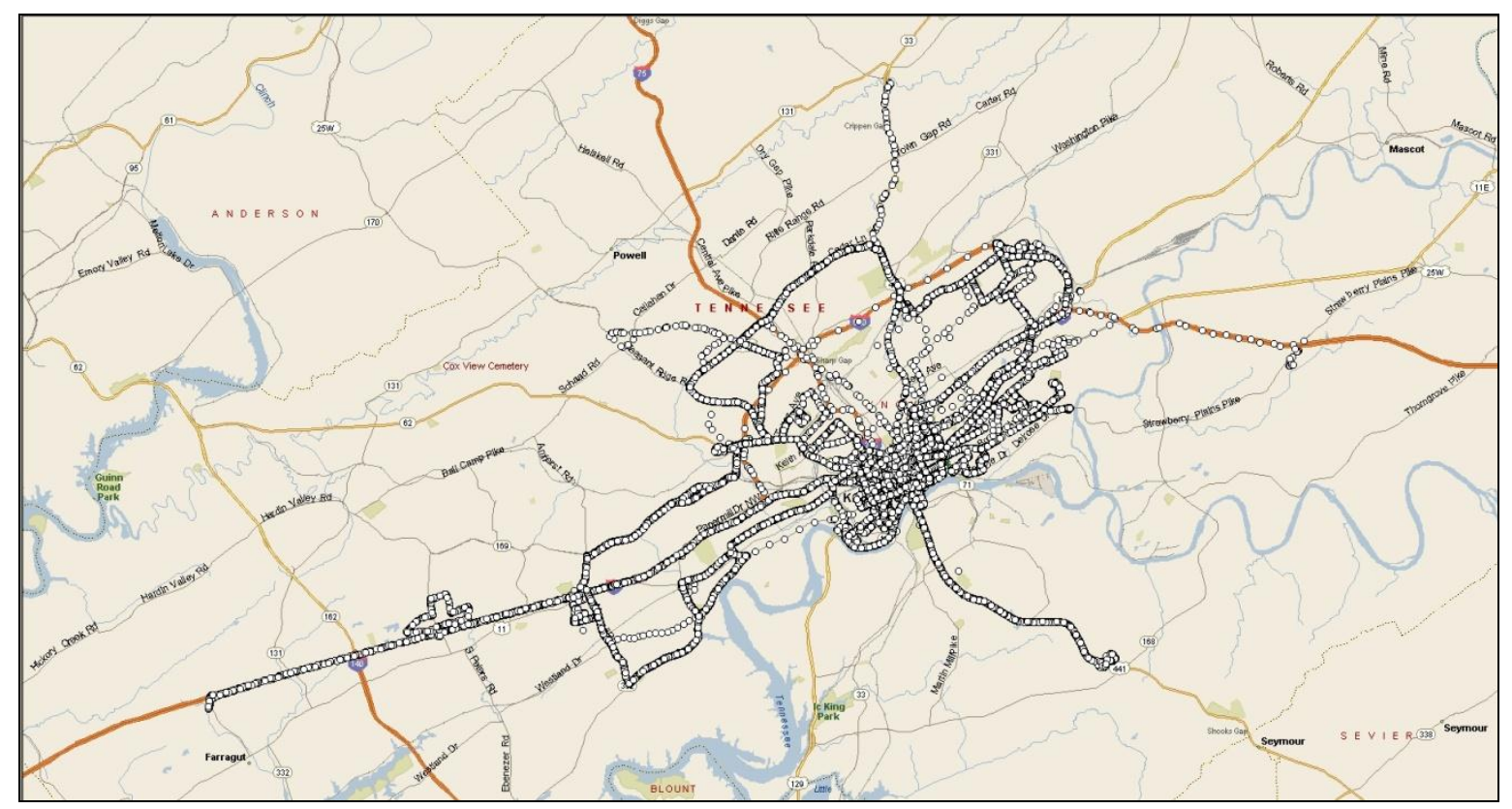

Figure 27. Transit Bus 3 (Vehicle 6) Coverage Area

Table 7 and Table 8 present a summary of these statistics for each one of the three participating combination trucks and the three transit buses, respectively. The tables also include information regarding fuel efficiencies for the entire data collection period, for each of the six vehicles, as well as their overall fuel efficiency. These calculations were made using fuel consumption information obtained from the vehicles' data bus (last row in Table 7 and Table 8 ) and include both the fuel consumed while the vehicle is moving as well as the fuel spent while idling.

Table 7. General Statistics for the Combination Trucks

\begin{tabular}{|c|r|r|r|r|}
\hline & \multicolumn{3}{|c|}{ MTDC Vehicle } & \multirow{2}{*}{$\begin{array}{c}\text { Grand } \\
\text { Total }\end{array}$} \\
\cline { 2 - 4 } & \multicolumn{1}{|c|}{$\mathbf{1}$} & \multicolumn{1}{c|}{$\mathbf{2}$} & \multicolumn{1}{c|}{$\mathbf{3}$} & \multicolumn{1}{c|}{} \\
\hline Distance Traveled [miles] & 18,483 & 6,556 & 20,397 & 45,435 \\
\hline Total Travel Time [hrs] & 565 & 190 & 556 & 1,311 \\
\hline Average Speed [mph] & 32.7 & 34.5 & 36.7 & 34.7 \\
\hline Average Moving Speed [mph] & 42.4 & 41.4 & 42.7 & 42.4 \\
\hline Total Fuel Consumed [gal] & 2,568 & 841 & 2,597 & 6,006 \\
\hline Overall Fuel Efficiency* [mpg] & 7.197 & 7.794 & 7.853 & 7.565 \\
\hline
\end{tabular}

*Computed using vehicle data bus information on fuel consumption, and integration of vehicle speed over time 
Table 8. General Statistics for the Transit Buses

\begin{tabular}{|c|c|c|c|c|}
\hline & \multicolumn{3}{|c|}{ MTDC Vehicle } & \multirow{2}{*}{$\begin{array}{l}\text { Grand } \\
\text { Total }\end{array}$} \\
\hline & 4 & 5 & 6 & \\
\hline Distance Traveled [miles] & 23,174 & 7,736 & 18,531 & 49,442 \\
\hline Total Travel Time [hrs] & 2,481 & 847 & 1,878 & 5,205 \\
\hline Average Speed [mph] & 9.3 & 9.1 & 9.9 & 9.5 \\
\hline Average Moving Speed [mph] & 19.3 & 19.7 & 19.6 & 19.4 \\
\hline Total Fuel Consumed [gal] & 5,432 & 1,764 & 4,176 & 11,372 \\
\hline Overall Fuel Efficiency* [mpg] & 4.267 & 4.386 & 4.437 & 4.348 \\
\hline
\end{tabular}

The information collected was used to generate distributions of idling time and idling fuel consumed as percentage of total time and total fuel consumed, respectively. Seven intervals of time were considered, ranging from 0-5 minutes (i.e., the vehicle was idling -vehicle static and engine running- for less than five minutes) to more than 240 minutes (4 hours); with the short intervals corresponding to idling due to traffic conditions (i.e., delays at traffic lights, congestion, and bus dwelling time at bus stops) and the largest one to overnight parking and garage idling. The idling information is presented in Table 9 and Table 10, for the combination trucks and the transit buses, respectively.

For the combination trucks (Table 9), the largest proportion of idling time (61\%) and fuel consumed (50\%) while idling correspond to idling intervals that last between 0 and 5 minutes(i.e., traffic congestion and delay at traffic signals). This is followed by intervals of 5 to 10 minutes of idling time (25\% of idling time and $25 \%$ of fuel consumption while idling), and by the 15 to 60 minute time interval. The latter is mostly idling while stopping for a delivery. The 180 to 240 minute interval is proportionally low in terms of idling time (1.5\%) but ranks third (12\%) in terms of fuel consumed while idling.

The transit buses also spent most of their idling time (31\%) in congestion and bus dwelling stops (0 to 5 minute idling interval) which also consumes the largest proportion of fuel spent while idling (see Table 10). However, as opposed to the combination trucks, the transit buses spent $26 \%$ of their idling time in intervals that are larger than 4 hours consuming also about $26 \%$ of the fuel spent while idling. These large idling times were observed mostly at the parking lot while the vehicles were waiting to start a trip.

Table 9. Distributions of Time Spent and Fuel Consumed while Idling (Combination Trucks)

\begin{tabular}{|c|r|r|r|r|r|r|}
\hline \multirow{2}{*}{$\begin{array}{c}\text { Idling Interval } \\
\text { [min] }\end{array}$} & \multicolumn{3}{|c|}{ Time Spent } & \multicolumn{3}{c|}{ Fuel Consumed } \\
\cline { 2 - 7 } & & $\begin{array}{c}\text { Percent } \\
\text { of Total } \\
\text { Idling } \\
\text { Time }\end{array}$ & $\begin{array}{c}\text { Percent } \\
\text { of Total } \\
\text { Time }\end{array}$ & [gal] & $\begin{array}{r}\text { Percent } \\
\text { of Total } \\
\text { Idling } \\
\text { Fuel }\end{array}$ & $\begin{array}{c}\text { Percent } \\
\text { of Total } \\
\text { Fuel }\end{array}$ \\
\hline $\mathbf{0 - 5}$ & 142.1 & 61.4 & 10.8 & 63.1 & 50.1 & 1.1 \\
\hline $\mathbf{5 - 1 0}$ & 59.1 & 25.5 & 4.5 & 32.3 & 25.6 & 0.5 \\
\hline $\mathbf{1 5 - 6 0}$ & 15.0 & 6.5 & 1.1 & 10.6 & 8.4 & 0.2 \\
\hline $\mathbf{6 0 - 1 2 0}$ & 2.2 & 0.9 & 0.2 & 1.4 & 1.1 & 0.0 \\
\hline $\mathbf{1 2 0 - 1 8 0}$ & 9.6 & 4.2 & 0.7 & 3.0 & 2.4 & 0.1 \\
\hline $\mathbf{1 8 0 - 2 4 0}$ & 3.4 & 1.5 & 0.3 & 15.5 & 12.3 & 0.3 \\
\hline $\mathbf{2 4 0 +}$ & 0.0 & 0.0 & 0.0 & 0.0 & 0.0 & 0.0 \\
\hline TOTAL & 231.4 & 100 & & 125.9 & 100 & \\
\hline
\end{tabular}


Table 10. Distributions of Time Spent and Fuel Consumed while Idling (Transit Buses)

\begin{tabular}{|c|r|r|r|r|r|r|}
\hline \multirow{2}{*}{$\begin{array}{c}\text { Idling Interval } \\
\text { [min] }\end{array}$} & \multicolumn{3}{|c|}{ Time Spent } & \multicolumn{3}{c|}{ Fuel Consumed } \\
\cline { 2 - 7 } & [hrs] & $\begin{array}{c}\text { Percent } \\
\text { of Total } \\
\text { Idling } \\
\text { Time }\end{array}$ & $\begin{array}{c}\text { Percent } \\
\text { of Total } \\
\text { Time }\end{array}$ & Igal] & $\begin{array}{r}\text { Percent } \\
\text { of Total } \\
\text { Idling } \\
\text { Fuel }\end{array}$ & $\begin{array}{r}\text { Percent } \\
\text { of Total } \\
\text { Fuel }\end{array}$ \\
\hline $\mathbf{0 - 5}$ & 808.4 & 30.7 & 1.6 & 782.9 & 30.6 & 6.9 \\
\hline $\mathbf{5 - 1 0}$ & 314.8 & 11.9 & 0.6 & 304.7 & 11.9 & 2.7 \\
\hline $\mathbf{1 5 - 6 0}$ & 285.3 & 10.8 & 0.6 & 281.1 & 11.0 & 2.5 \\
\hline $\mathbf{6 0 - 1 2 0}$ & 277.2 & 10.5 & 0.6 & 251.7 & 9.8 & 2.2 \\
\hline $\mathbf{1 2 0 - 1 8 0}$ & 150.9 & 5.7 & 0.3 & 154.5 & 6.0 & 1.4 \\
\hline $\mathbf{1 8 0 - 2 4 0}$ & 116.8 & 4.4 & 0.2 & 111.2 & 4.3 & 1.0 \\
\hline $\mathbf{2 4 0 +}$ & 682.2 & 25.9 & 1.4 & 670.8 & 26.2 & 5.9 \\
\hline TOTAL & $2,635.6$ & 100 & & $2,556.9$ & 100 & \\
\hline
\end{tabular}

\subsubsection{Class-7 Vehicle Fuel Efficiency}

Using the information collected in the project, it is possible to compute the fuel efficiencies of combination trucks and transit buses. The overall fuel efficiencies, that is the fuel efficiencies (FEs) computed taking into consideration idling times, as well as the moving FEs (i.e., fuel consumed only when the vehicle was moving) are presented in Table 11 and Table 12 for the three combination trucks and three transit buses, respectively. The tables also present the percentage difference between overall and moving FEs for each vehicle. Notice that for the transit buses the percentage difference between overall and moving FEs is much higher (between 10 and 20 times higher) than that of the combination trucks. Some of this difference can be attributed to differences between different types of vehicles (transmission type, age, etc.), but most is due to idling. While the total miles traveled by the two sets of vehicles during the year-long project is almost the same, about $10 \%$ larger for the transit buses than for the combination trucks, the former consumed almost 20 times more fuel than the latter while idling (see Table 7 to Table 10). Using the moving FE information, it is possible to see that the FEs are very different, being almost $40 \%$ higher for the combination trucks than for the transit buses.

Table 11. Overall and Moving Fuel Efficiency - Combination Trucks

\begin{tabular}{|c|r|r|r|}
\hline MTDC Vehicle & $\begin{array}{c}\text { Overall FE } \\
{[\mathrm{mpg}]}\end{array}$ & \multicolumn{1}{c|}{$\begin{array}{c}\text { Moving FE } \\
\text { [mpg] }\end{array}$} & $\begin{array}{c}\text { Percent } \\
\text { Difference }\end{array}$ \\
\hline 1 & 7.197 & 7.407 & 2.91 \\
\hline 2 & 7.794 & 7.925 & 1.68 \\
\hline 3 & 7.853 & 7.974 & 1.54 \\
\hline All Vehicles & 7.565 & 7.727 & 2.14 \\
\hline
\end{tabular}


Table 12. Overall and Moving Fuel Efficiency - Transit Buses

\begin{tabular}{|c|r|r|r|}
\hline MTDC Vehicle & $\begin{array}{c}\text { Overall FE } \\
{[\mathrm{mpg}]}\end{array}$ & \multicolumn{1}{c|}{$\begin{array}{c}\text { Moving FE } \\
{[\mathrm{mpg}]}\end{array}$} & $\begin{array}{c}\text { Percent } \\
\text { Difference }\end{array}$ \\
\hline 4 & 4.267 & 5.488 & 28.64 \\
\hline $\mathbf{5}$ & 4.386 & 5.773 & 31.63 \\
\hline 6 & 4.437 & 5.698 & 28.41 \\
\hline All Vehicles & 4.348 & 5.609 & 29.01 \\
\hline
\end{tabular}

\subsection{WEIGHT ESTIMATION}

\subsubsection{Weight Estimation Model}

The weight prediction models described in section 3.2.1 were applied to all of the information collected during the project by the combination trucks. Figure 28 to Figure 30 present the distributions of measured steer and drive axle weights and modeled truck weight for vehicles 1 to 3 , respectively. Those distributions were built by counting trips (i.e., from a stop to the next stop, with stopping times larger than 10 minutes) that presented the same payload level, and using 1,000 lbs payloads bins. Notice that there were a few trips where the weight of the tractor was about 14,000 lbs; those were trips within the warehouse parking area in which the tractor did not have any trailer attached. Notice also that the three combination trucks had different total vehicle weight distributions. This is mostly due the fact that each of the three vehicles, in general, repeated the same delivery routes week after week, and also to the length of the routes. For example, Combination Truck 3 carried, on average, heavier payloads (see Table 13) since it traveled further from the warehouse than the other two vehicles (see Figure 22 to Figure 24), visiting each delivery stop with less frequency.

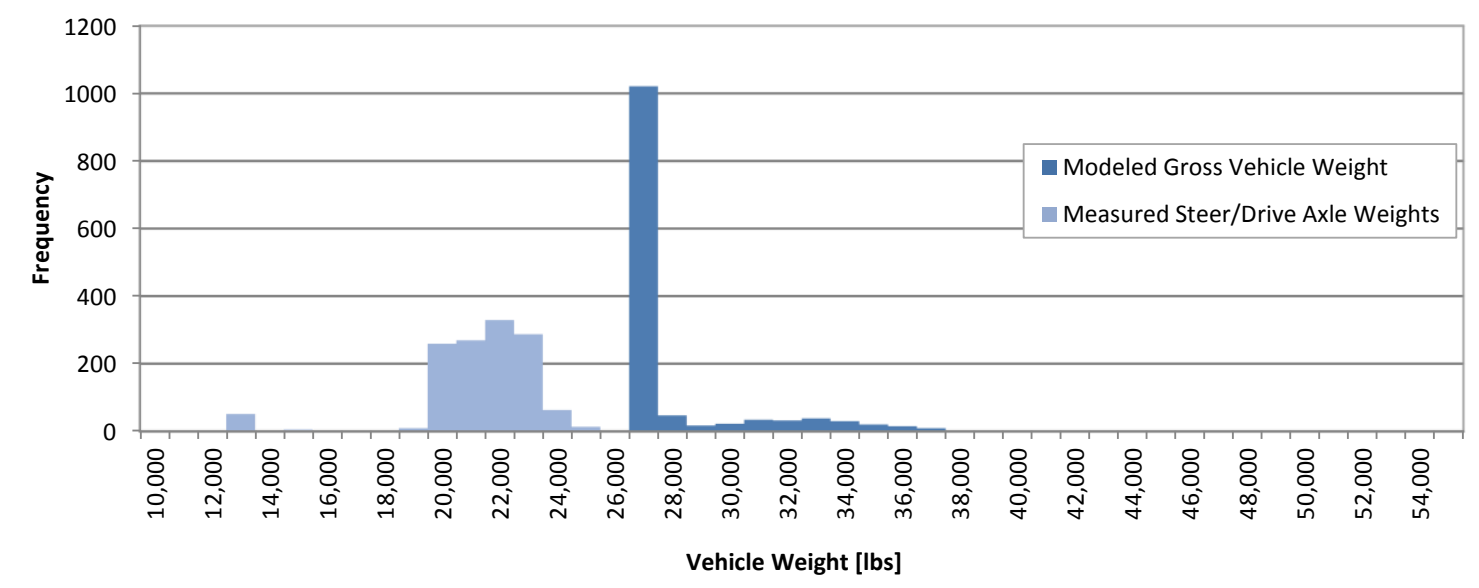

Figure 28. Combination Truck 1 (Vehicle 1) Frequency Distributions of Measured Steer/Drive Axle Weights and Modeled Gross Vehicle Weight 


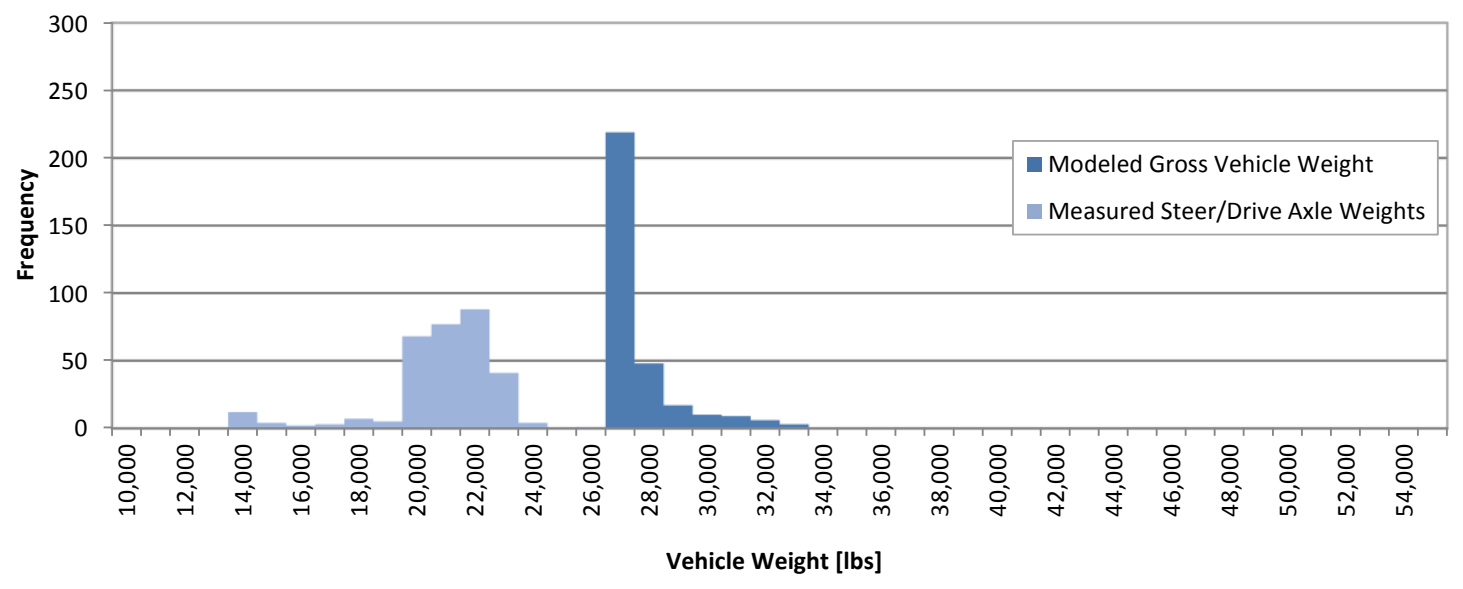

Figure 29. Combination Truck 2 (Vehicle 2) Frequency Distributions of Measured Steer/Drive Axle Weights and Modeled Gross Vehicle Weight

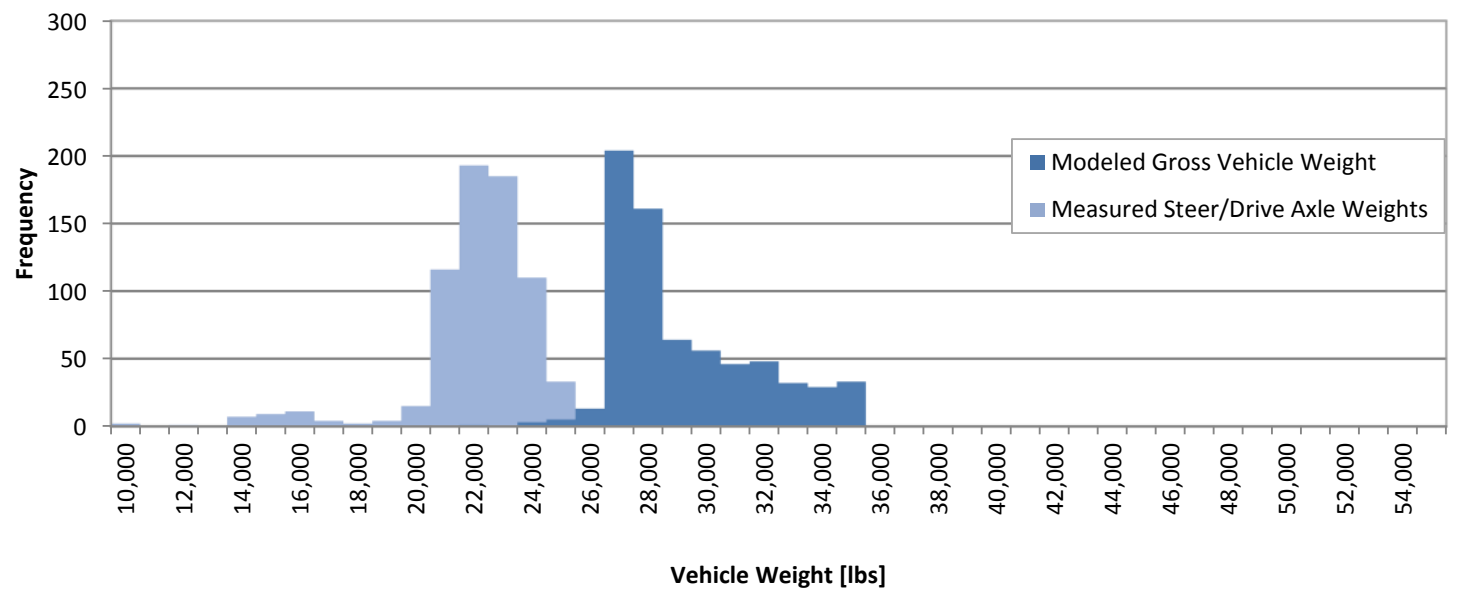

Figure 30. Combination Truck 3 (Vehicle 3) Frequency Distributions of Measured Steer/Drive Axle Weights and Modeled Gross Vehicle Weight 
In the case of the transit buses, it was not necessary to develop a weight prediction model since the vehicles were equipped with the Air-Weigh device which measured total vehicle weight all of the time. Total vehicle weight distributions were built in a similar manner as those of the combination trucks discussed above. These distributions are presented in Figure 31 to Figure 33. Transit Buses 1 and 2 had a similar, and skewed, total vehicle weight distributions with a higher frequency of trips with heavier payloads (i.e., more passengers) than the mode of the distribution (i.e., the value that occurs the most frequently in the distribution). In contrast, Transit Bus 3 showed a more normally distributed (i.e., lessskewed) total-vehicle weight with almost similar number of trips with lighter and heavier payloads. This is the result of the different routes that these vehicles followed during the year-long data collection effort.

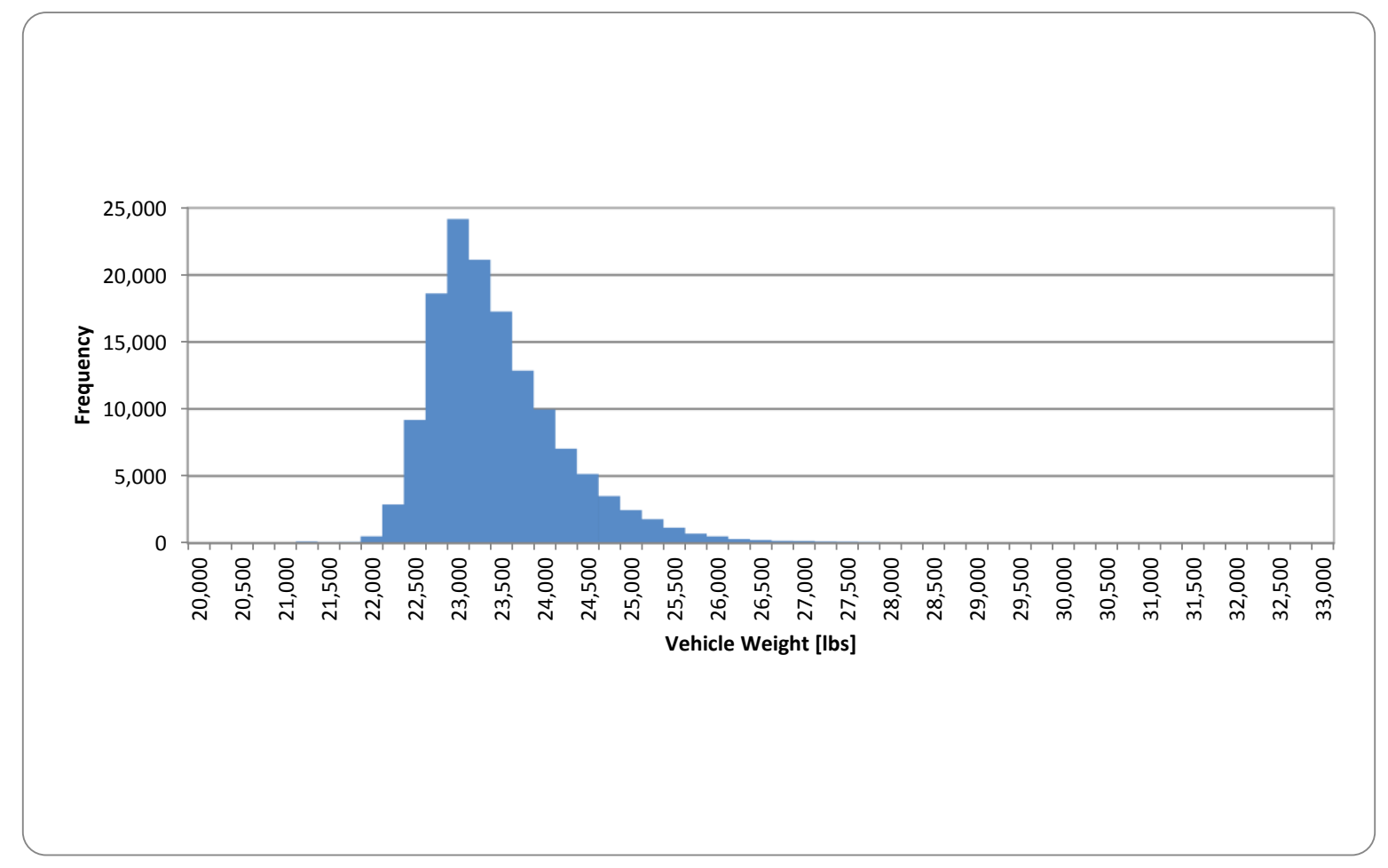

Figure 31. Transit Bus 1 (Vehicle 4) Frequency Distribution of Gross Vehicle Weight 


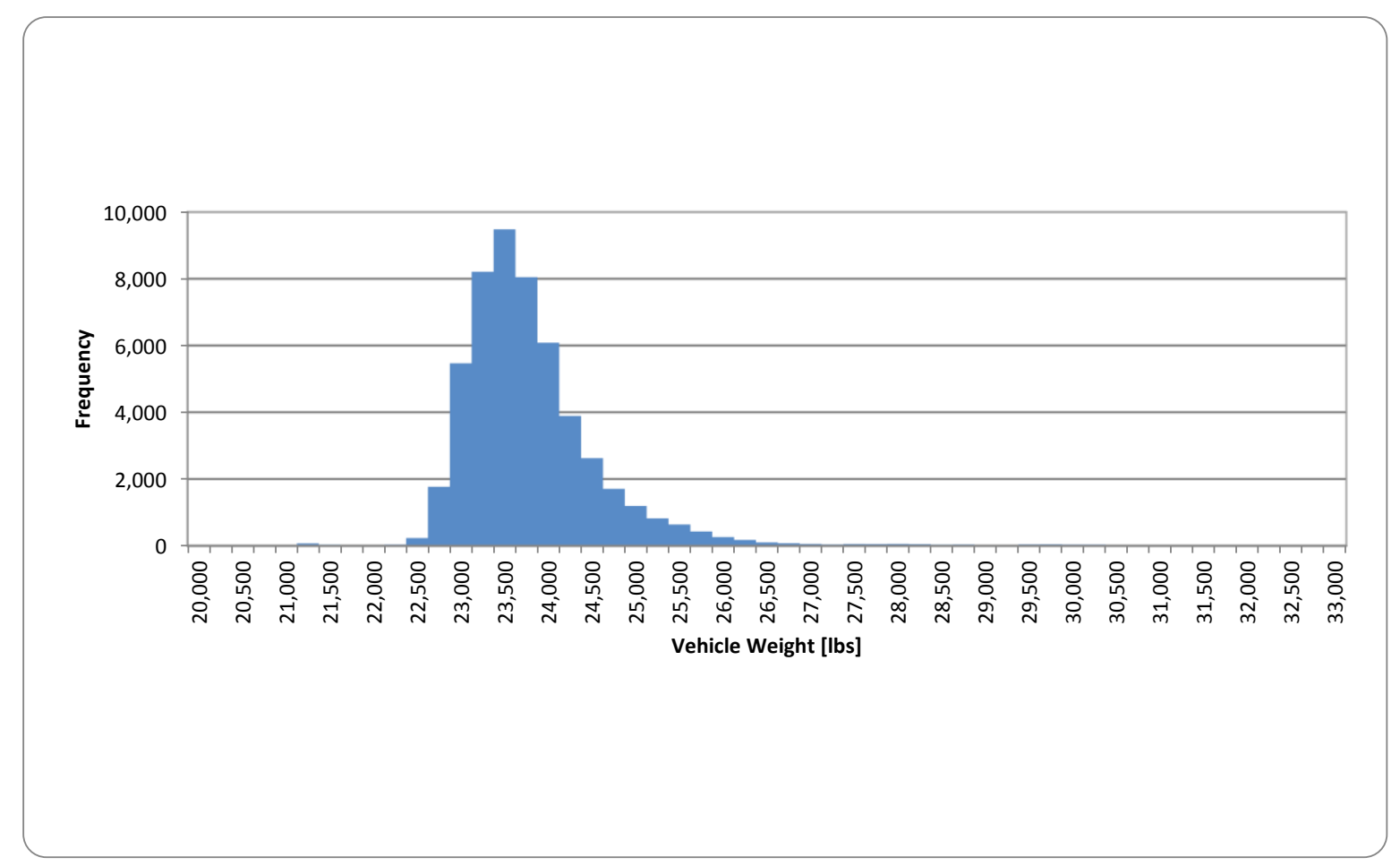

Figure 32. Transit Bus 2 (Vehicle 5) Frequency Distribution of Gross Vehicle Weight

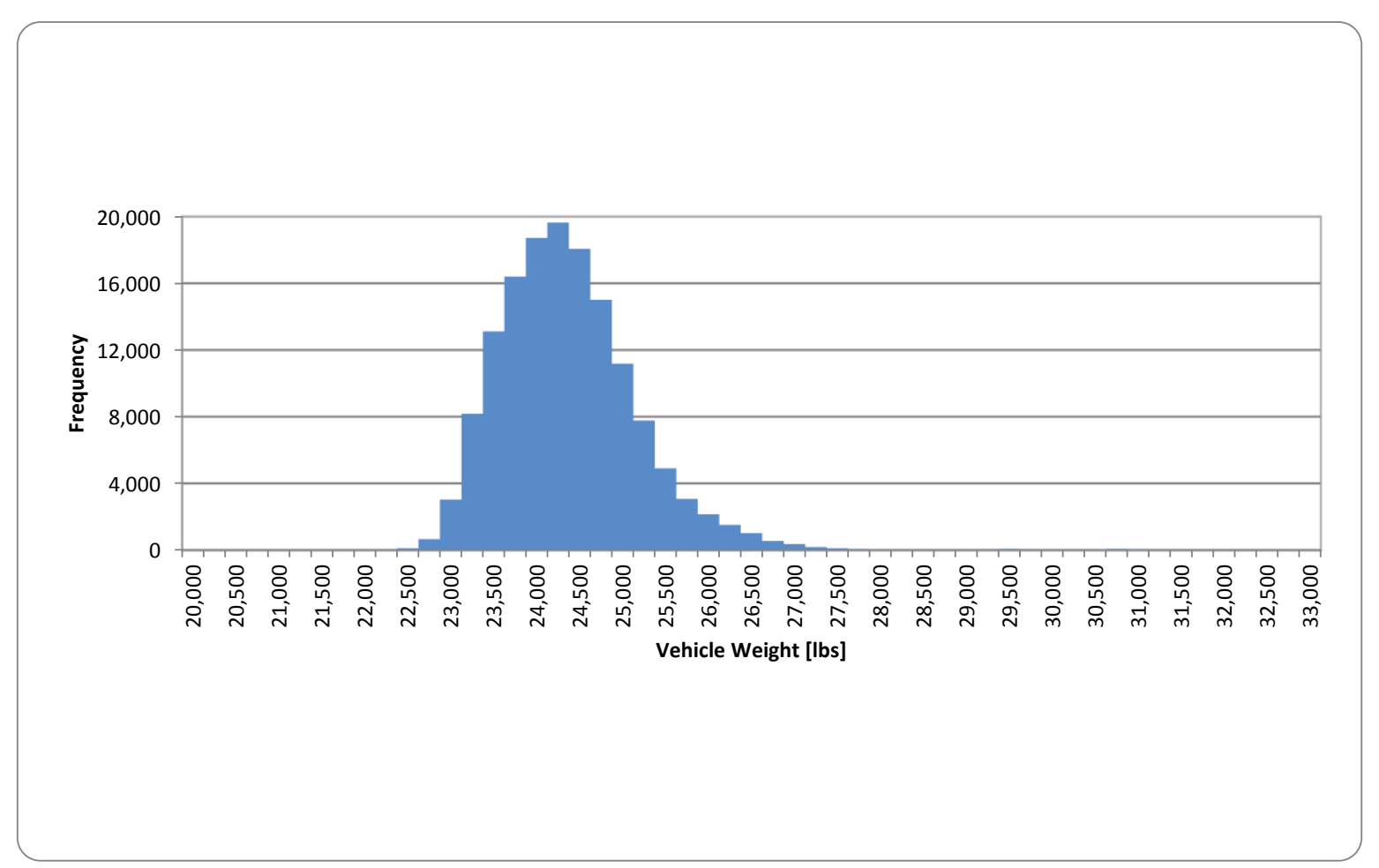

Figure 33. Transit Bus 3 (Vehicle 6) Frequency Distribution of Gross Vehicle Weight 


\subsubsection{Effect of Vehicle Weight on Fuel Efficiency}

One very important variable affecting the fuel efficiency of any vehicle is its weight or payload. The previous sub-sections showed FE measurements that were obtained without controlling for any particular variable since it could be assumed that any of the participating vehicles would experience the same situations (terrain, weather, payload, etc.) over the course of the year-long experiment. In this sub-section the weight of the vehicle is introduced as a controlling variable to study how the payload affects the FE of combination trucks and transit buses.

In order to do this, the search engine of the DCGenT Prototype was used to parse the data by vehicle load level. For the combination trucks, the vehicle weight was divided into four categories: 1) Tractor and Empty Trailer (this category also includes tractor-only trips), 2) Light Load (total vehicle weight between 27,000 lbs and 31,999 lbs); 3) Medium Load (total vehicle weight between 32,000 lbs and 37,999 lbs); and 4) Heavy Load (total vehicle weight above 38,000 lbs). In the case of the transit buses the vehicle weight was divided into three categories: 1) Empty Bus (vehicle weight less than 22,500 lbs), 2) Light Fare (total vehicle weight between 22,500 lbs and 25,499 lbs); and 3) Heavy Fare (total vehicle weight above 25,500 lbs).

Table 13 and Table 14 present summary statistics of distribution of payloads grouped under the weight levels described above for the combination trucks and transit buses, respectively. The tables show the average weight in each load level category as well as the percentage of the time that the vehicle carried a load that was within that category. On average, the combination trucks weighted between 27,700 lbs to $29,000 \mathrm{lbs}$ and the buses weighed between 23,000 lbs and 23,800 lbs. Very few trips in the "tractor and empty trailer" category were observed (these were mostly trips within the warehouse area). Most of the trips for vehicle $2(98 \%)$ were at a light or medium load, while for vehicle 3, almost $70 \%$ of the trips were at the medium and high level loads as explained above.

Table 13. Combination Truck Weight-Level Statistics

\begin{tabular}{|c|c|c|c|c|c|c|c|c|c|c|}
\hline \multirow{3}{*}{ MTDC Vehicle } & \multicolumn{10}{|c|}{ Load Level } \\
\hline & \multicolumn{2}{|c|}{ Any Payload } & \multicolumn{2}{|c|}{$\begin{array}{l}\text { Tractor and } \\
\text { Empty Trailer }\end{array}$} & \multicolumn{2}{|c|}{ Light Load } & \multicolumn{2}{|c|}{ Medium Load } & \multicolumn{2}{|c|}{ Heavy Load } \\
\hline & $\begin{array}{c}\text { Percent } \\
\text { of } \\
\text { Time }\end{array}$ & $\begin{array}{c}\text { Average } \\
\text { Weight } \\
\text { [lbs] }\end{array}$ & $\begin{array}{l}\text { Percent } \\
\text { of Time }\end{array}$ & $\begin{array}{c}\text { Average } \\
\text { Weight } \\
\text { [lbs] }\end{array}$ & $\begin{array}{l}\text { Percent } \\
\text { of Time }\end{array}$ & $\begin{array}{c}\text { Average } \\
\text { Weight } \\
\text { [lbs] }\end{array}$ & $\begin{array}{l}\text { Percent } \\
\text { of Time }\end{array}$ & $\begin{array}{c}\text { Average } \\
\text { Weight } \\
\text { [lbs] }\end{array}$ & $\begin{array}{l}\text { Percent } \\
\text { of Time }\end{array}$ & $\begin{array}{c}\text { Average } \\
\text { Weight } \\
\text { [lbs] }\end{array}$ \\
\hline 1 & 100.0 & 28,166 & $0.0^{*}$ & 0 & 70.2 & 26,557 & 15.5 & 29,691 & 14.3 & 34,398 \\
\hline 2 & 100.0 & 27,713 & $0.0^{*}$ & 0 & 58.7 & 26,838 & 40.6 & 28,894 & 0.7 & 32,460 \\
\hline 3 & 100.0 & 29,038 & 0.4 & 22,785 & 32.4 & 26,313 & 48.0 & 29,132 & 19.2 & 33,528 \\
\hline
\end{tabular}

Transit Buses 2 and 3 (vehicles 5 and 6) operated almost $90 \%$ of the time with a light fare as defined above. Vehicle 6 also had the largest average weight, indicating that this was the vehicle that carried the largest number of passengers (on average). Transit Bus 1 (vehicle 4), on the other hand spent almost 30\% of the time at an empty level. This was mostly due to idling at the parking garage. This vehicle had the lowest overall FE out of the three buses (see Table 12). 
Table 14. Transit Buses Weight-Level Statistics

\begin{tabular}{|c|r|r|r|r|r|r|r|r|}
\hline \multirow{2}{*}{ MTDC Vehicle } & \multicolumn{9}{|c|}{ Load Level } \\
\cline { 2 - 9 } & \multicolumn{2}{|c|}{ Any Fare } & \multicolumn{2}{|c|}{ Empty } & \multicolumn{2}{c|}{ Light Fare } & \multicolumn{2}{c|}{ Heavy Fare } \\
\cline { 2 - 9 } & $\begin{array}{c}\text { Percent } \\
\text { of Time }\end{array}$ & $\begin{array}{c}\text { Average } \\
\text { Weight } \\
{[\text { [lbs] }}\end{array}$ & $\begin{array}{c}\text { Percent } \\
\text { of Time }\end{array}$ & $\begin{array}{c}\text { Average } \\
\text { Weight } \\
{[\text { [lbs] }}\end{array}$ & $\begin{array}{c}\text { Percent } \\
\text { of Time }\end{array}$ & $\begin{array}{c}\text { Average } \\
\text { Weight } \\
{[\text { lbs] }}\end{array}$ & $\begin{array}{c}\text { Percent } \\
\text { of Time }\end{array}$ & $\begin{array}{c}\text { Average } \\
\text { Weight } \\
{[\mathrm{lbs}]}\end{array}$ \\
\hline 4 & 100.0 & 22,958 & 28.1 & 22,198 & 69.9 & 23,176 & 2.0 & 26,027 \\
\hline 5 & 100.0 & 23,230 & 7.6 & 22,257 & 89.7 & 23,222 & 2.7 & 26,296 \\
\hline 6 & 100.0 & 23,854 & 3.2 & 22,274 & 88.7 & 23,734 & 8.1 & 25,775 \\
\hline
\end{tabular}

To generate the distribution of fuel efficiency under the payload levels described above, 10-mile segments were considered for which the fuel efficiency was computed and counted as one observation. Table 15 presents the results of this analysis for the combination trucks. Under each load level category, the column labeled "Average FE" contains the average of the fuel efficiencies across all the 10-mile segments, with the column immediately to the right presenting the standard deviation of the distribution. Overall, and as expected, the FE decreases as the payload increases. However, because the payload categories are very narrow and low (those combination trucks never made any trips that were above 42,000 lbs, far below the legal weight limit for Class-7 trucks), the variation of the FE is not significant.

Table 15. Fuel Efficiency as a Function of Load Level - Combination Trucks

\begin{tabular}{|c|r|r|r|r|r|r|r|r|}
\hline \multirow{2}{*}{ MTDC Vehicle } & \multicolumn{4}{|c|}{ Load Level } & \multicolumn{2}{|c|}{ Heavy Load } \\
\cline { 2 - 10 } & \multicolumn{2}{|c|}{$\begin{array}{c}\text { Tractor with } \\
\text { Empty Trailer }\end{array}$} & \multicolumn{2}{|c|}{ Light Load } & \multicolumn{2}{c|}{ Medium Load } & \multicolumn{2}{c|}{$\mid$} \\
\cline { 2 - 10 } & $\begin{array}{c}\text { Average } \\
\text { FE [mpg] }\end{array}$ & $\begin{array}{c}\text { Standard } \\
\text { Deviation } \\
{[\mathrm{mpg}]}\end{array}$ & $\begin{array}{c}\text { Average } \\
\text { FE [mpg] }\end{array}$ & $\begin{array}{c}\text { Standard } \\
\text { Deviation } \\
{[\mathrm{mpg}]}\end{array}$ & $\begin{array}{c}\text { Average } \\
\text { FE [mpg] }\end{array}$ & $\begin{array}{c}\text { Standard } \\
\text { Deviation } \\
{[\mathrm{mpg}]}\end{array}$ & $\begin{array}{c}\text { Average } \\
\text { FE [mpg] }\end{array}$ & $\begin{array}{c}\text { Standard } \\
\text { Deviation } \\
{[\mathrm{mpg}]}\end{array}$ \\
\hline 1 & \#N/A* & \#N/A* & 7.520 & 1.020 & 7.191 & 1.021 & 7.091 & 1.065 \\
\hline $\mathbf{2}$ & \#N/A* & \#N/A* & 8.013 & 1.422 & 7.838 & 1.328 & 6.944 & 1.261 \\
\hline 3 & 8.299 & 1.708 & 8.345 & 1.715 & 7.667 & 1.470 & 7.904 & 1.452 \\
\hline All Vehicles & 8.299 & 1.708 & 7.997 & 1.493 & 7.574 & 1.345 & 7.532 & 1.351 \\
\hline
\end{tabular}

The distributions of FEs for each load-level and all the three vehicles combined are presented in graphical form in Figure 34. For the majority of the combination truck trips, the load level was light and medium (i.e., between 27,000 lbs and 38,000 lbs). 


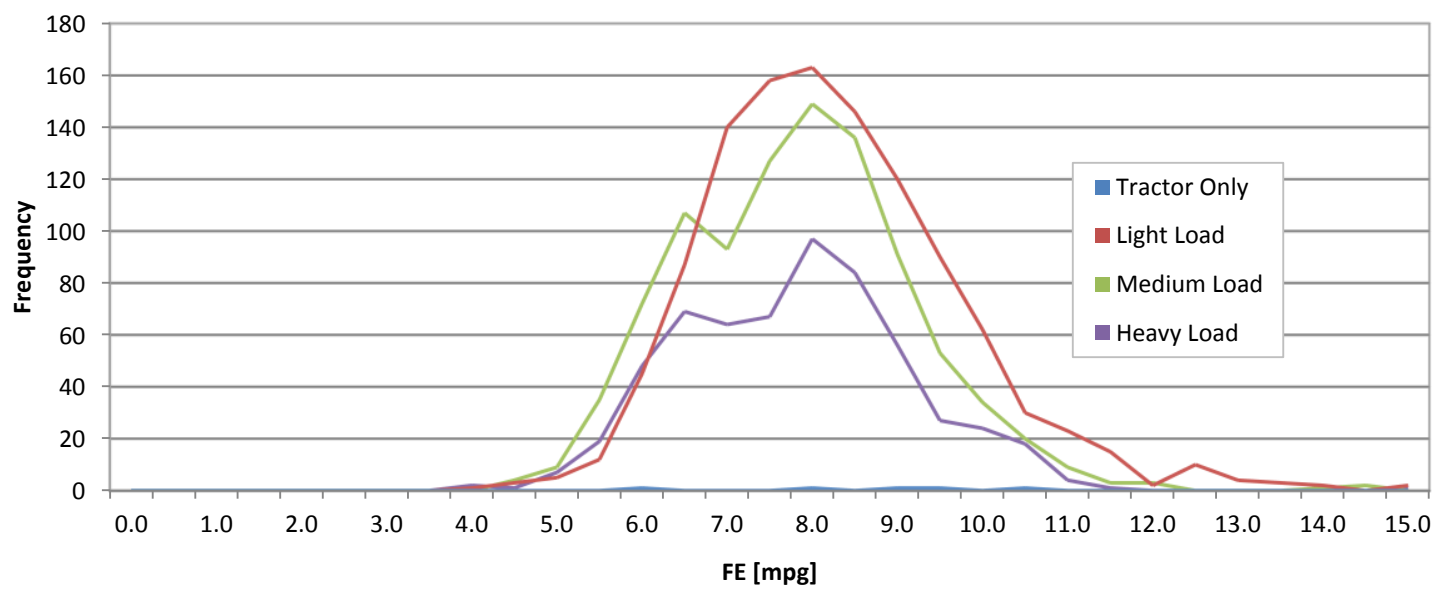

Figure 34. Fuel Efficiency Frequency Distributions as a Function of Load Level - Combination Trucks

In the case of the Transit Buses (see Table 16) the relationship between FE and vehicle weight is not as it would be predicted (i.e., decreasing FE with increasing payload). This is due to several factors. First, when the vehicle is empty (lowest payload) it spends a considerable amount time idling as discussed previously. This results in very low FEs for this load-level category. At the other end of the spectrum, the highway routes (express routes) are the ones that carry the largest number of passenger. The buses on these routes spent very little dwelling time (there are fewer bus stops) and also encounter the least number of traffic lights. This results in FEs that are higher than regular surface street routes (light fare) with many more stops (bus stops and traffic signals). Notice that in Table 16, vehicle 4 has a very low standard deviation for the Heavy Fare case. This was due to the fact that there were only two observations for that case.

Table 16. Fuel Efficiency as a Function of Load Level - Transit Buses

\begin{tabular}{|c|r|r|r|r|r|r|}
\hline \multirow{2}{*}{ MTDC Vehicle } & \multicolumn{4}{|c|}{ Load Level } \\
\cline { 2 - 7 } & \multicolumn{2}{|c|}{ Empty } & \multicolumn{2}{c|}{ Light Fare } & \multicolumn{2}{c|}{ Heavy Fare } \\
\cline { 2 - 7 } & Average $[\mathrm{mpg}]$ & $\begin{array}{c}\text { Standard } \\
\text { Deviation } \\
{[\mathrm{mpg}]}\end{array}$ & $\begin{array}{c}\text { Average } \\
\text { FE [mpg] }\end{array}$ & $\begin{array}{c}\text { Standard } \\
\text { Deviation } \\
{[\mathrm{mpg}]}\end{array}$ & $\begin{array}{c}\text { Average } \\
\text { FE [mpg] }\end{array}$ & $\begin{array}{c}\text { Standard } \\
\text { Deviation } \\
{[\mathrm{mpg}]}\end{array}$ \\
\hline $\mathbf{4}$ & 3.261 & 1.751 & 4.941 & 1.191 & 3.433 & 0.084 \\
\hline $\mathbf{5}$ & 4.465 & 1.368 & 4.724 & 1.656 & 7.412 & 0.980 \\
\hline $\mathbf{6}$ & 3.953 & 1.818 & 4.820 & 1.466 & 5.317 & 0.906 \\
\hline All Vehicles & 3.397 & 1.748 & 4.866 & 1.373 & 5.685 & 1.450 \\
\hline
\end{tabular}


The distributions of FEs for each load-level and all the three transit buses combined are presented in graphical form in Figure 35. For the majority of the transit bus trips, the load level was light fare (i.e., between $22,500 \mathrm{lbs}$ and 25,500 lbs).

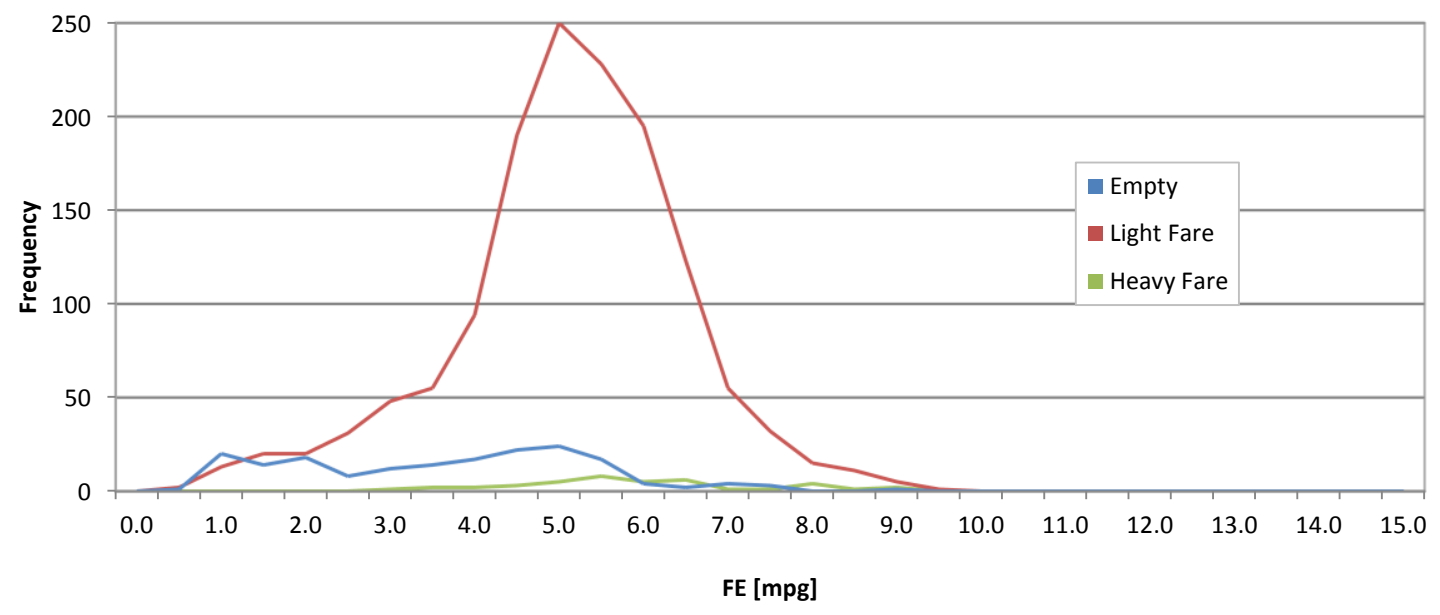

Figure 35. Fuel Efficiency Frequency Distributions as a Function of Load Level - Transit Buses

\subsection{CLASSIFICATION OF DUTY CYCLES}

\subsubsection{Transit Bus Highway Duty Cycles}

The data collected in this project can also be used to investigate the variability that may exist in duty cycles that are generated by the same vocation and that follow the same route. In order to do so, two sets of duty cycles collected from the data gathered by the transit buses were identified. The first set focused on freeway duty cycles. A $19 \mathrm{~km}$ segment of I-40 in Knoxville, Tennessee was selected (see Figure 36) and the corresponding duty cycles extracted from the database. This is a segment of urban highway that is used in several KAT bus routes in both the westbound and eastbound directions. For this study only the westbound direction was used. 


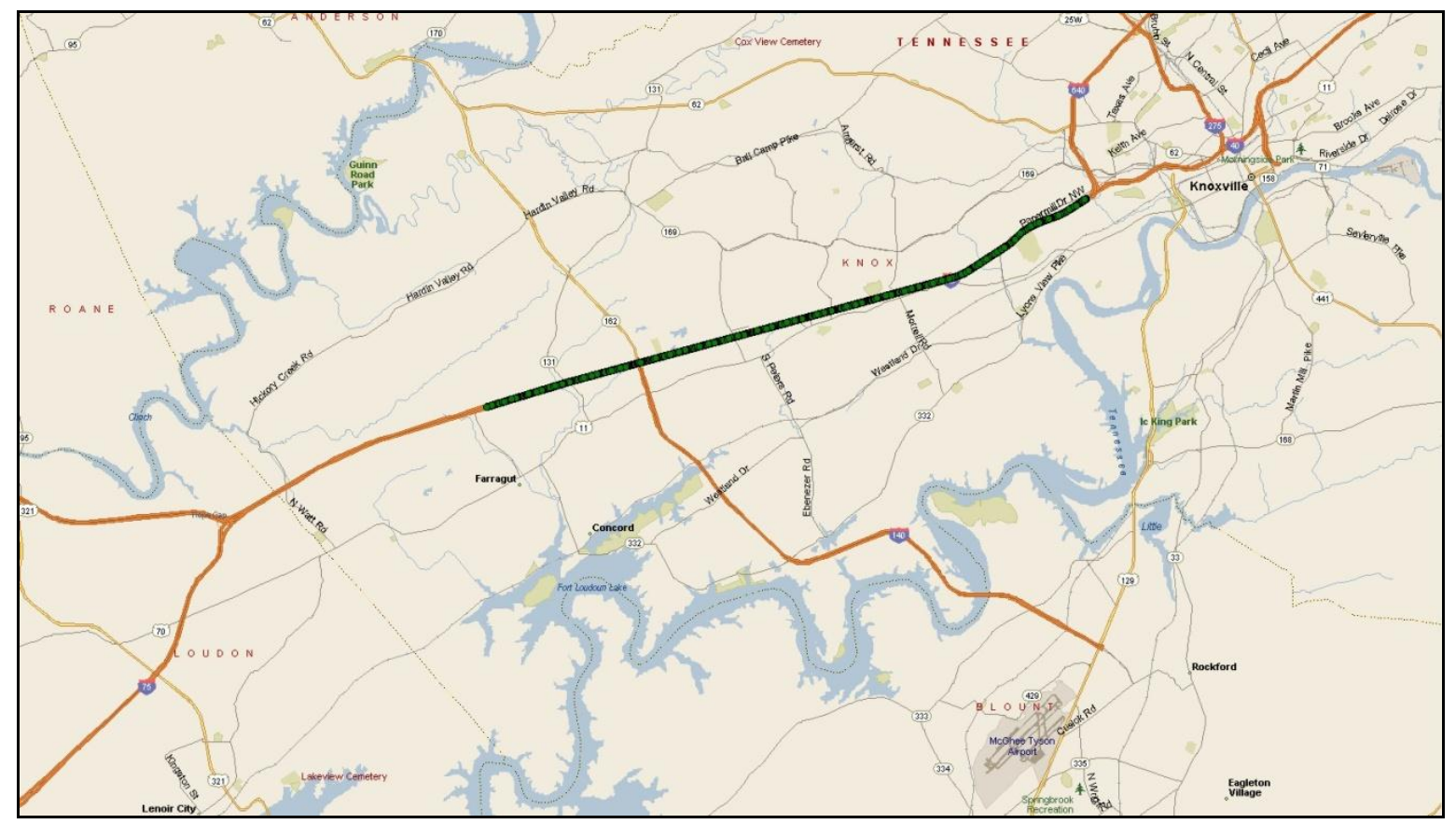

Figure 36. Transit Bus Highway Duty Cycle Route

Seventy-four highway duty cycles with the characteristics described above were selected. The majority of these duty cycles corresponded to the morning peak hour, with considerably fewer occurring during the afternoon peak hour (see Figure 37).

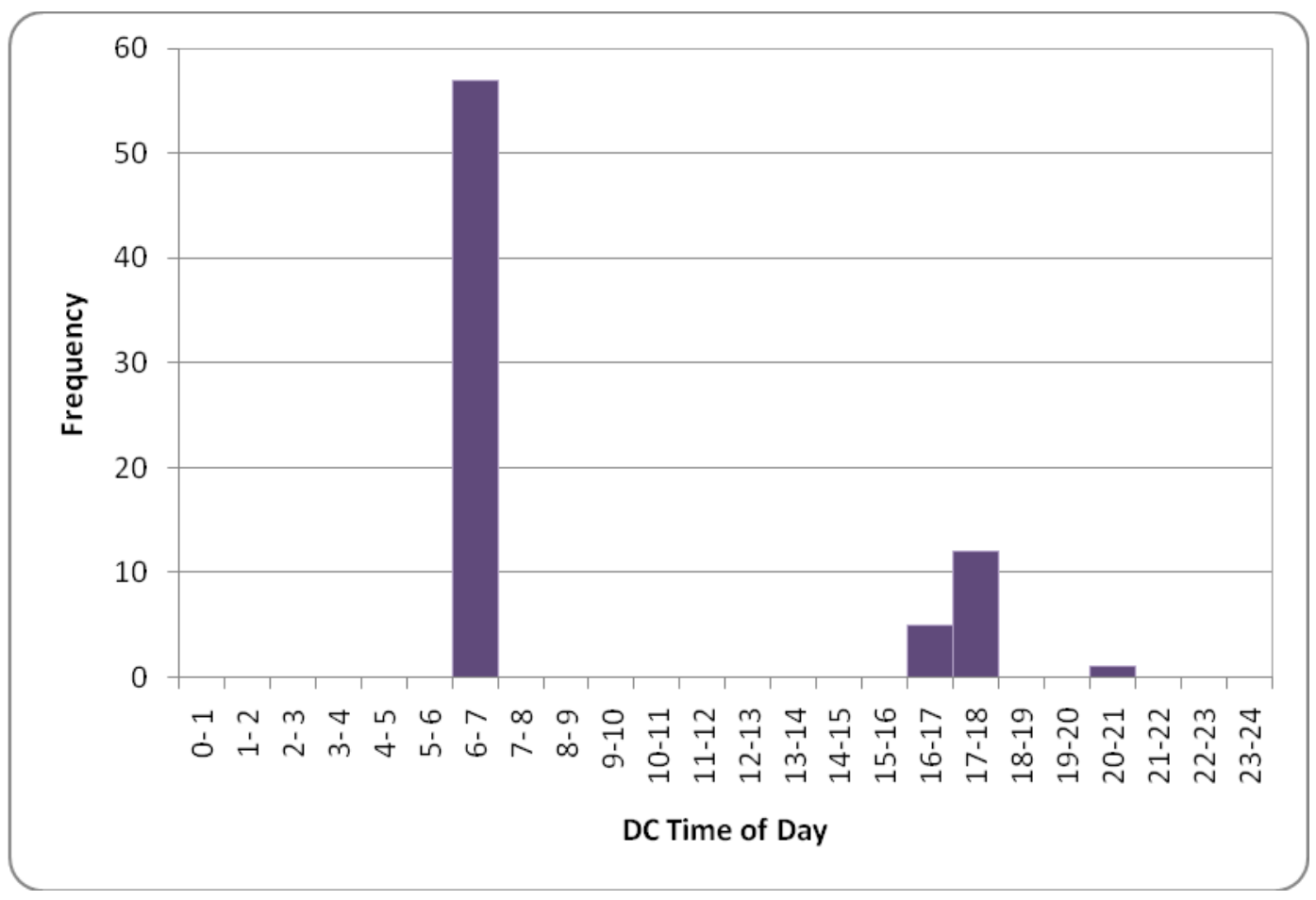

Figure 37. Transit Bus Highway Duty Cycle (DC) Time-of-Day Frequency Distribution 
The duration of the duty cycles ranged from 10.5 minutes to 27.6 minutes with an average duration of 12.6 minutes and a standard deviation of 2.71 minutes. Table 17 presents this and other statistics that define the distributions of these 74 highway duty cycles. One key statistic in the variability of the highway duty cycles is the distribution of minimum speed, which is directly related to congestion. Table 17 shows that there were significant variations in the minimum speed of the duty cycles. The minimum speed ranged from $0.0 \mathrm{mph}$ to $56.5 \mathrm{mph}$ with an average of $41.5 \mathrm{mph}$ and a standard deviation of 16.2 $\mathrm{mph}$. This statistic presented the highest ratio of standard deviation to mean, indicating a high variability. Graphical representations of the distributions of the duty cycle durations and minimum speeds are shown in Figure 38 and Figure 39, respectively.

Table 17. Transit Bus Highway Duty Cycle Distribution Statistics

\begin{tabular}{|c|r|r|r|r|r|}
\hline $\begin{array}{c}\text { Duty Cycle Parameter Distribution } \\
\text { Statistics }\end{array}$ & Mean & $\begin{array}{c}\text { Standard } \\
\text { Deviation }\end{array}$ & Minimum & Maximum & $\begin{array}{c}\text { Number of } \\
\text { Observations }\end{array}$ \\
\hline Duration [min] & 12.67 & 2.71 & 10.51 & 27.64 & 74 \\
\hline Average Speed [mph] & 55.81 & 7.61 & 25.04 & 64.85 & 74 \\
\hline Standard Deviation in Speed [mph] & 5.04 & 5.90 & 1.39 & 26.21 & 74 \\
\hline Maximum Speed [mph] & 63.80 & 3.40 & 56.54 & 69.03 & 74 \\
\hline Minimum Speed [mph] & 41.52 & 16.16 & 0.00 & 56.50 & 74 \\
\hline
\end{tabular}

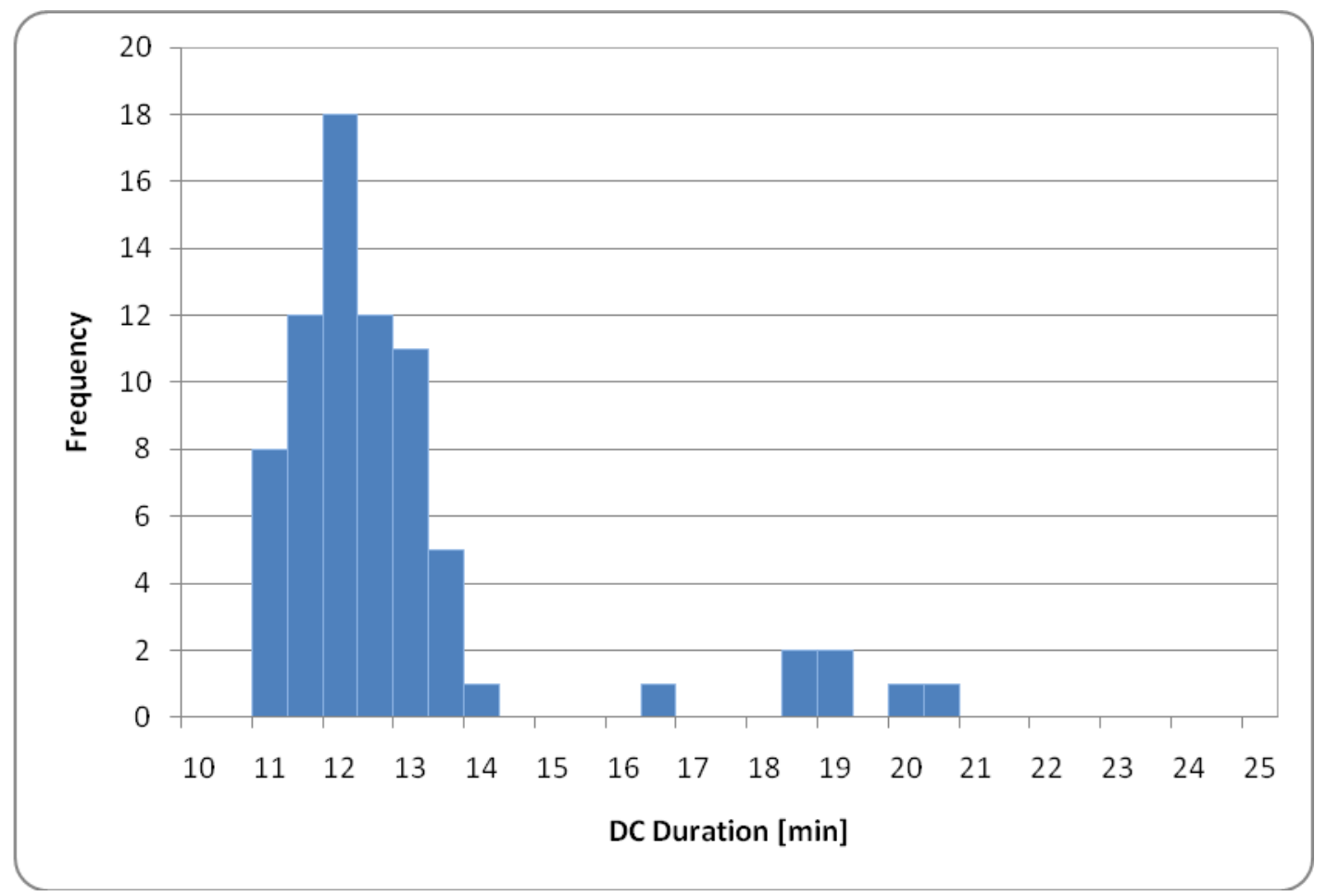

Figure 38. Transit Bus Highway Duty Cycle (DC) Duration Frequency Distribution 


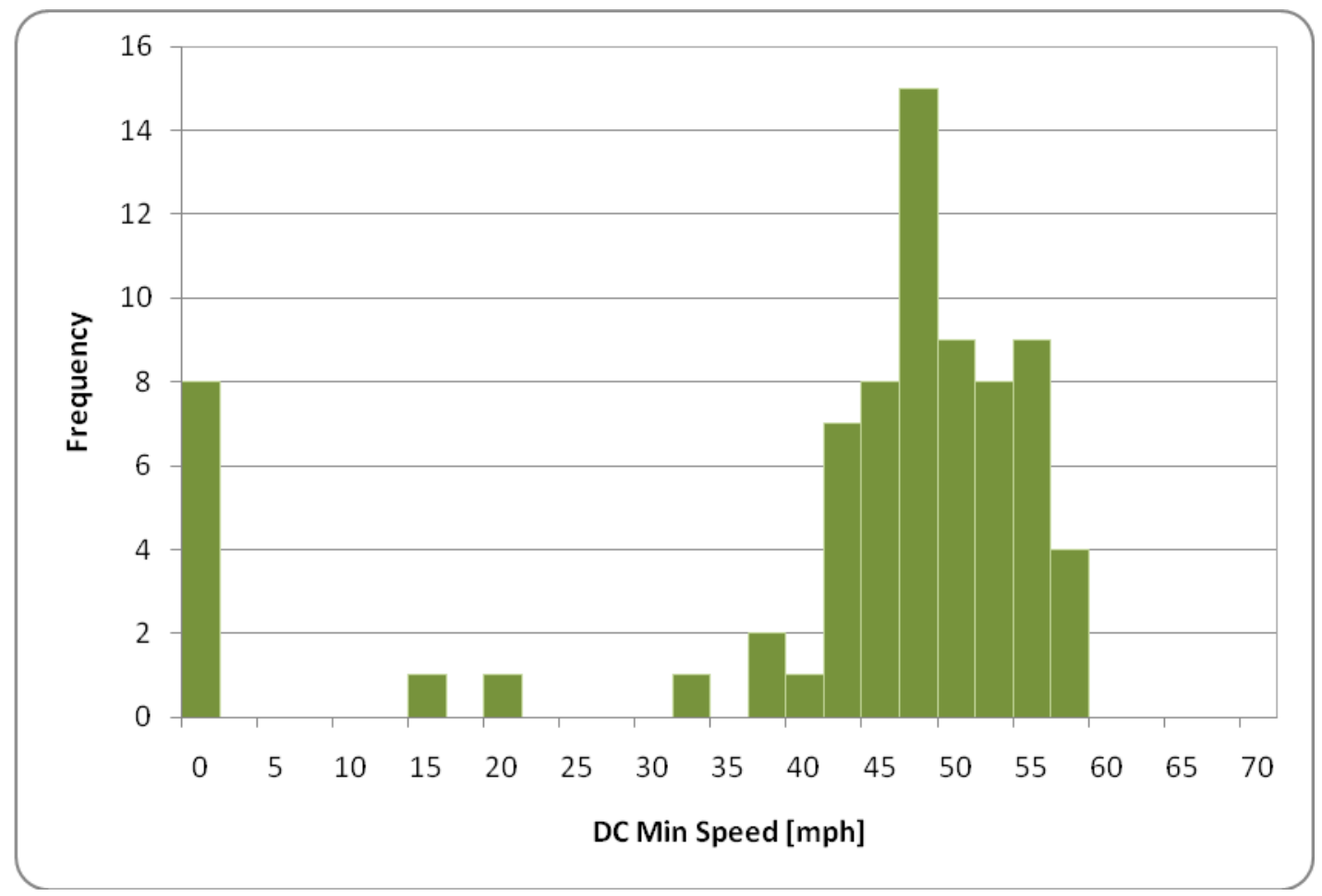

Figure 39. Transit Bus Highway Duty Cycle (DC) Minimum Speed Frequency Distribution

The 74 duty cycles were ranked in descending order using the duty cycle average speed as the sorting variable. Ten of these duty cycles, the top five and the bottom five were selected and graphed. The results are presented in Figure 40 and Figure 41, respectively. The top 5 duty cycles show very little variability in terms of cycle length and shape. The profile of these duty cycles follows the geometric characteristics of the highway in terms of speed, which in this case is almost free-flow speed (highway Level of Service A). In the case of the bottom five, there is a higher variability in terms of the duty cycle length and also in the shape of the duty cycles. The latter directly reflects the traffic conditions on the freeway.

Taking into account these considerations, and as a first approach for highway duty cycles, the variability in the duty cycle length provides a measure of the duty cycle variability. The speed is a function of the length of the highway segment and the duration of the duty cycle, so the variability of the speed will directly affect the variability of the duty cycle length. The duty cycle variability could be measured by the ratio of the standard deviation of the distribution of duty cycle lengths over the mean of that distribution. For the 74 duty cycles, this ratio is 0.214 (i.e., 2.71 minutes/12.7 minutes; see Table 17). For the five top and five bottom duty cycles shown in Figure 40 and Figure 41, the variability was computed at 0.06 and 0.357 , respectively. This indicates a skewed distribution of the duty-cycle variability, in which duty cycles are more likely to have high rather than low variability. Because the demand for public transit increases during peak hours, the bus frequency also increases during these periods. Therefore, a higher proportion of duty cycles were collected under higher traffic-congestion levels resulting in higher variability in the duty cycles. 


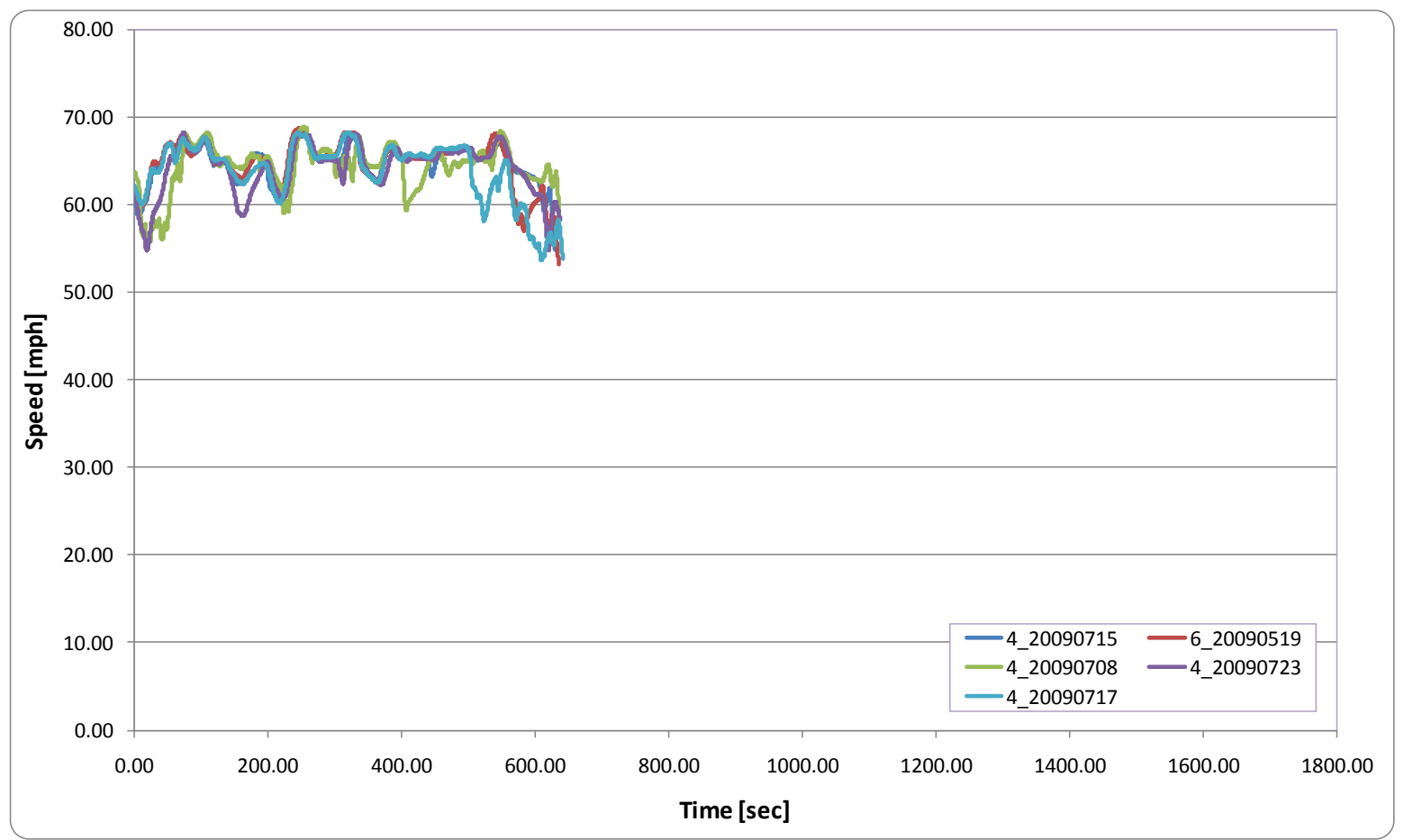

Figure 40. Top Five Maximum Average Speed Transit Bus Highway Duty Cycles

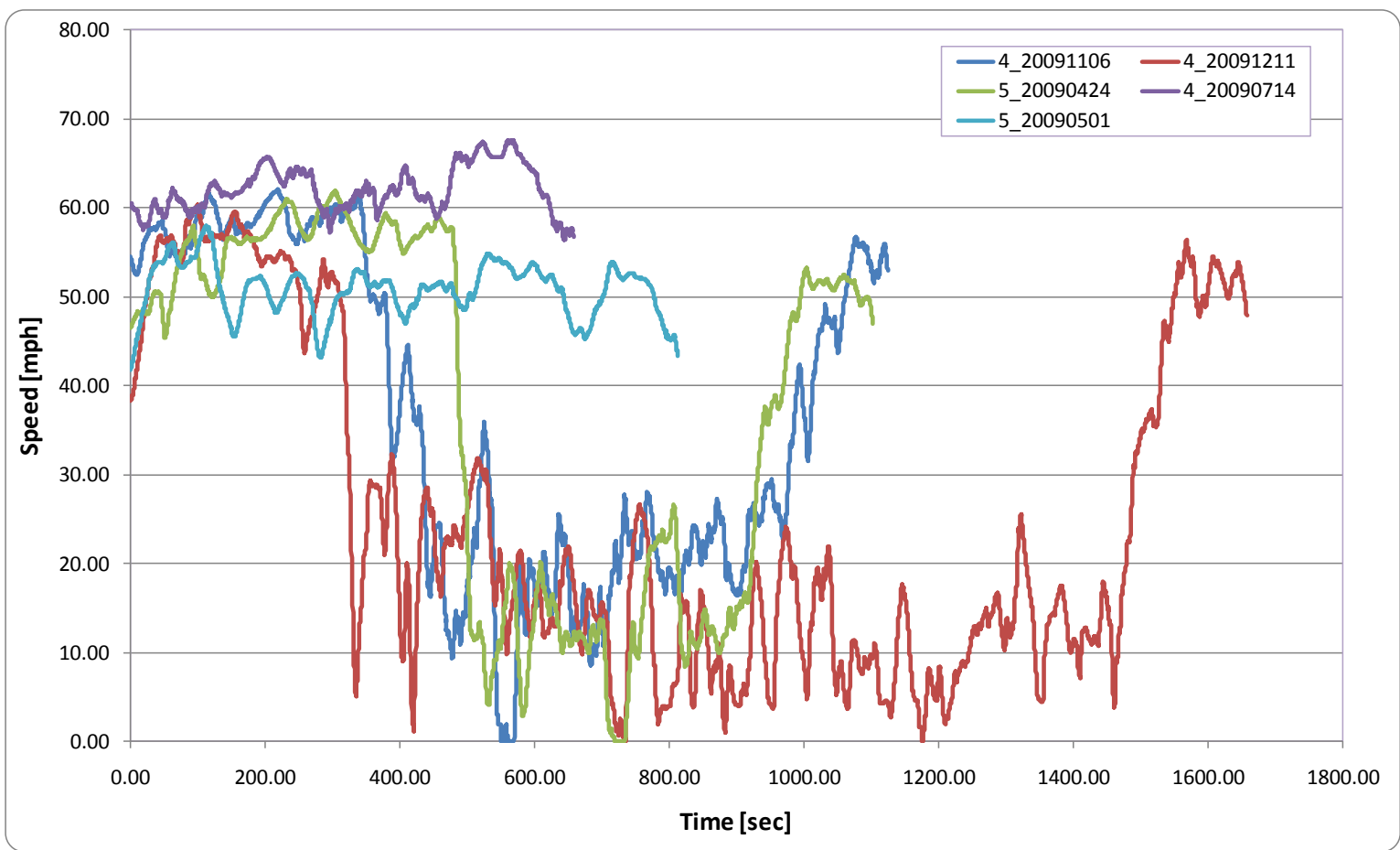

Figure 41. Bottom Five Maximum Average Speed Transit Bus Highway Duty Cycles 


\subsubsection{Transit Bus Surface-Street Duty Cycles}

The first set of duty cycles extracted from the MTDC database focused on surface street duty cycles. A $4.9 \mathrm{~km}$ loop in downtown Knoxville, Tennessee was selected and the corresponding duty cycles extracted from the database. Figure 42 shows this transit bus route.

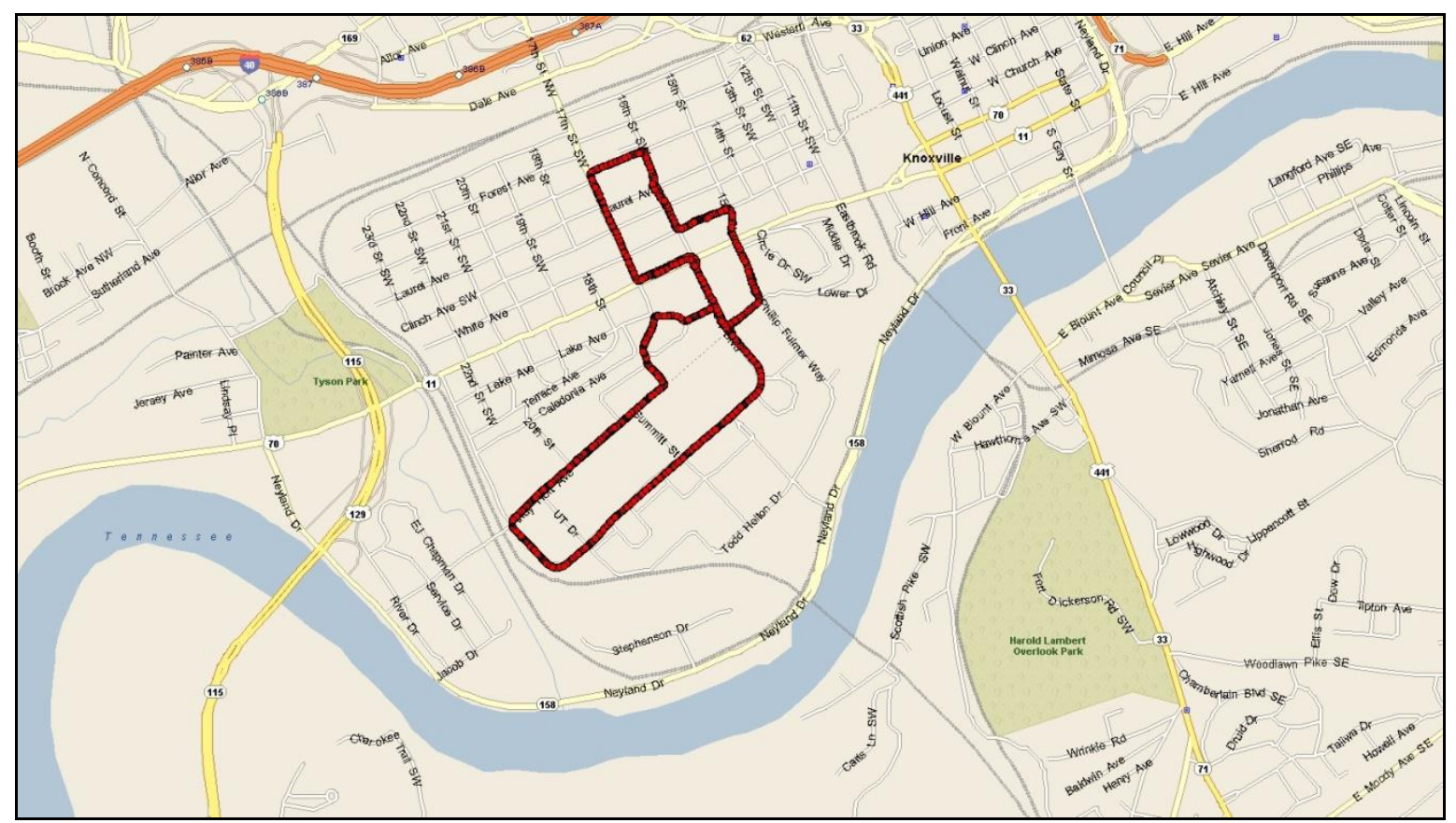

Figure 42. Transit Bus Surface Street Duty Cycle Route

Thirty-eight surface street duty cycles were selected. The duration length of the duty cycles ranged from 10.9 minutes to 22.8 minutes with an average duration of 18.4 minutes and a standard deviation of 3.29 minutes. Table 18 presents this and other statistics that define the distributions of these 38 highway duty cycles. Notice that, as opposed to freeway duty cycles, in all of the surface street duty cycles the minimum speed was $0.0 \mathrm{mph}$ (i.e., vehicle stationary). A graphical representation of the distribution of the duty cycle lengths is shown in Figure 43.

Table 18. Transit Bus Surface Street Duty Cycle Distribution Statistics

\begin{tabular}{|c|r|r|r|r|r|}
\hline $\begin{array}{c}\text { Duty Cycle Parameter } \\
\text { Distribution Statistics }\end{array}$ & Mean & $\begin{array}{c}\text { Standard } \\
\text { Deviation }\end{array}$ & Minimum & Maximum & $\begin{array}{c}\text { Number of } \\
\text { Observations }\end{array}$ \\
\hline Length [min] & 18.41 & 3.29 & 10.86 & 22.82 & 38 \\
\hline Average Speed [mph] & 9.23 & 1.89 & 2.50 & 13.90 & 38 \\
\hline Std Dev Speed [mph] & 7.49 & 0.90 & 5.42 & 9.34 & 38 \\
\hline Max Speed [mph] & 26.05 & 3.06 & 20.17 & 31.59 & 38 \\
\hline Min Speed [mph] & 0.00 & 0.00 & 0.00 & 0.00 & 38 \\
\hline
\end{tabular}




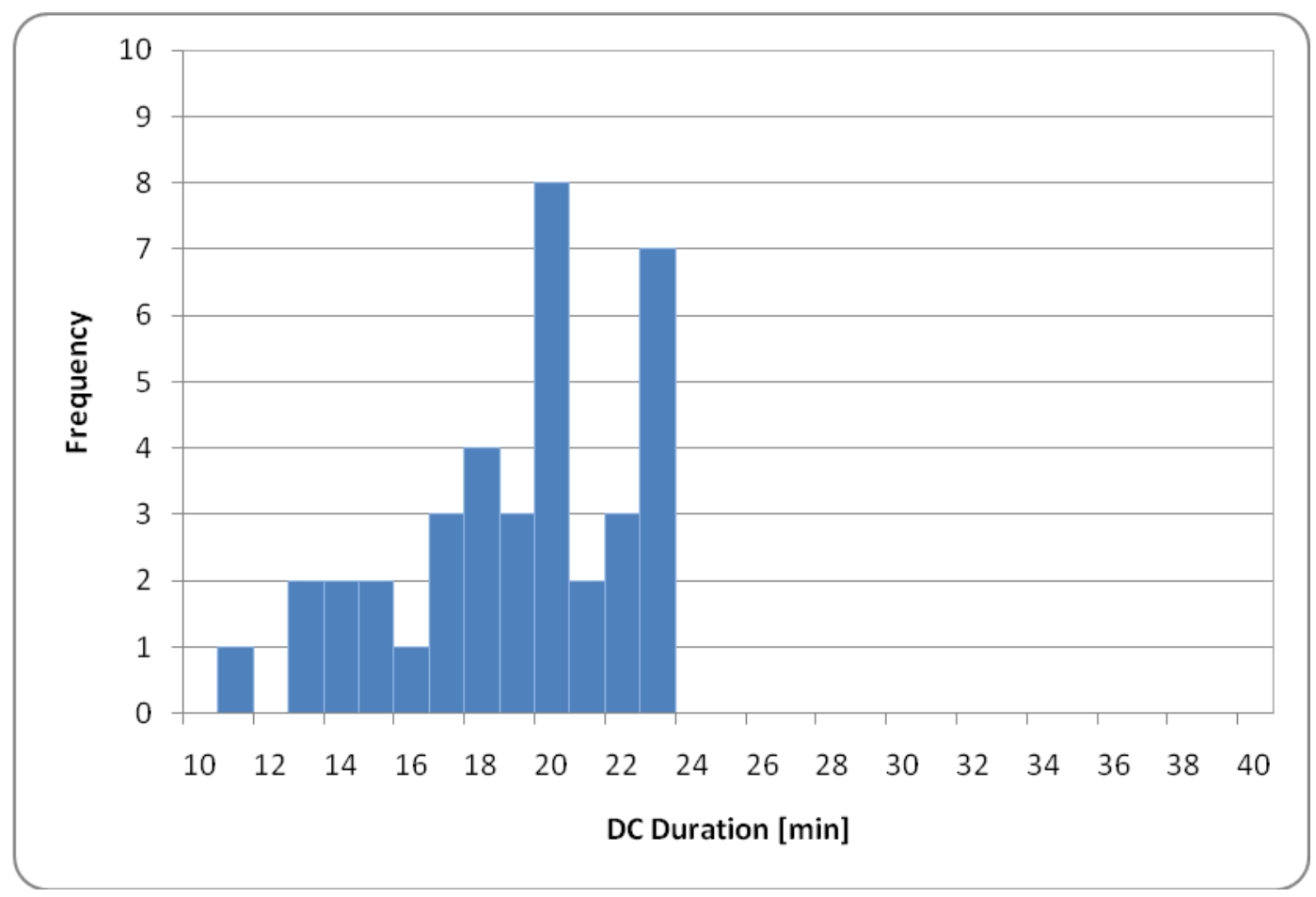

Figure 43. Transit Bus Surface Street Duty Cycle (DC) Duration Frequency Distribution

As was the case with the highway duty cycles, the 38 surface street duty cycles were arranged in descending order using the duty cycle average speed as the sorting variable. Ten of these duty cycles, the top five and the bottom five were selected and graphed. The results are presented in Figure 44 and Figure 45 , respectively. Similarly to the highway duty cycles, the top five surface street duty cycles do not show a large variability in terms of the duration of the cycle. In the case of the bottom five, there is a higher variability in duty cycle duration and also in the shape of the duty cycles. Using again the ratio of the standard deviation of the distribution of duty cycle duration over the mean of that distribution to estimate the variability of the duty cycles, the 38 duty cycles had a ratio equal to 0.179 (i.e., 3.29 minutes/18.4 minutes; see Table 18). For the five top and five bottom duty cycles shown in Figure 44 and Figure 45, the variability was computed at 0.134 and 0.262 , respectively. Using this measure, the highway duty cycles presented a higher variability than the surface street duty cycles (i.e., 0.214 vs. 0.179 ). 


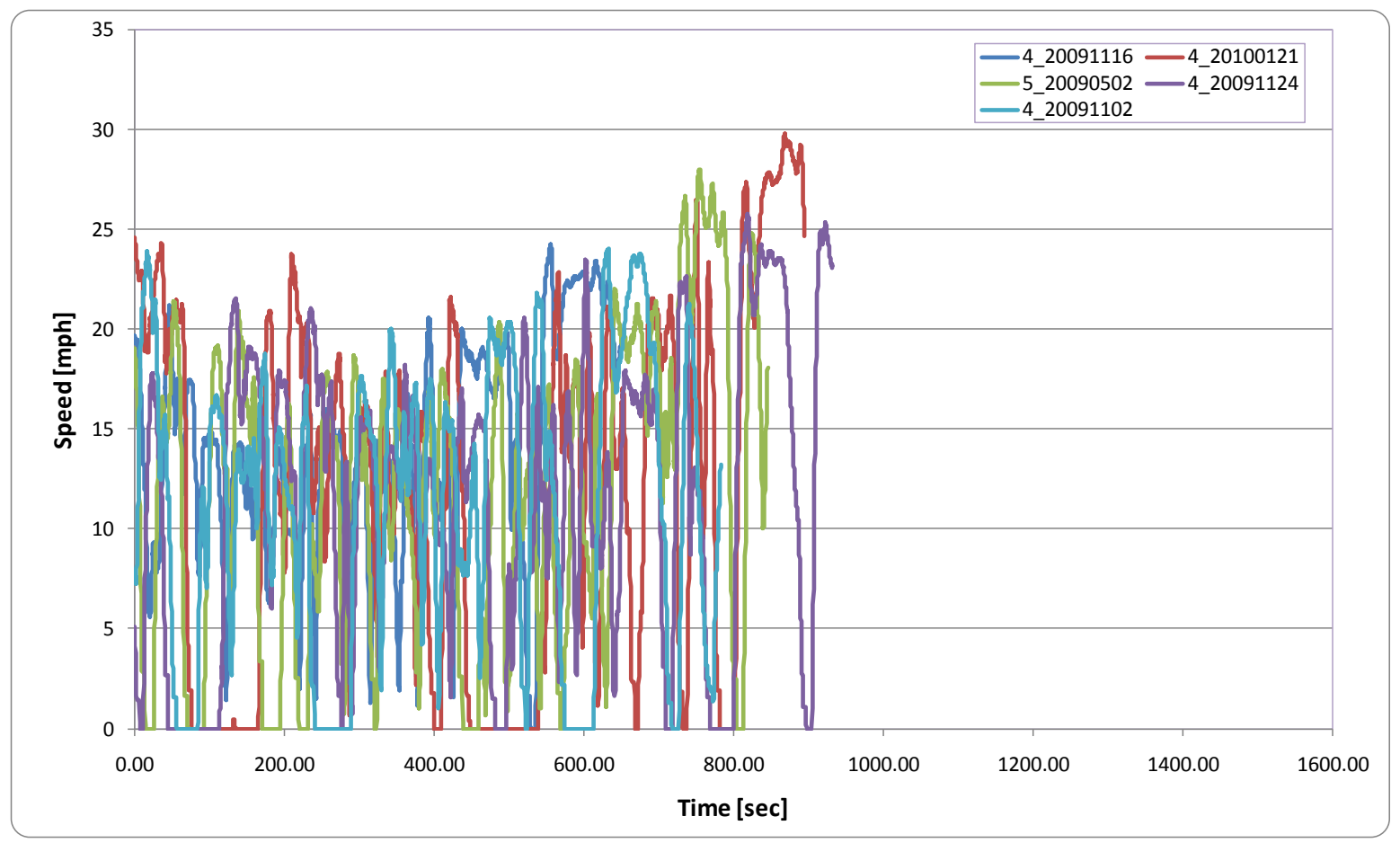

Figure 44. Top Five Maximum Average Speed Transit Bus Surface Street Duty Cycles

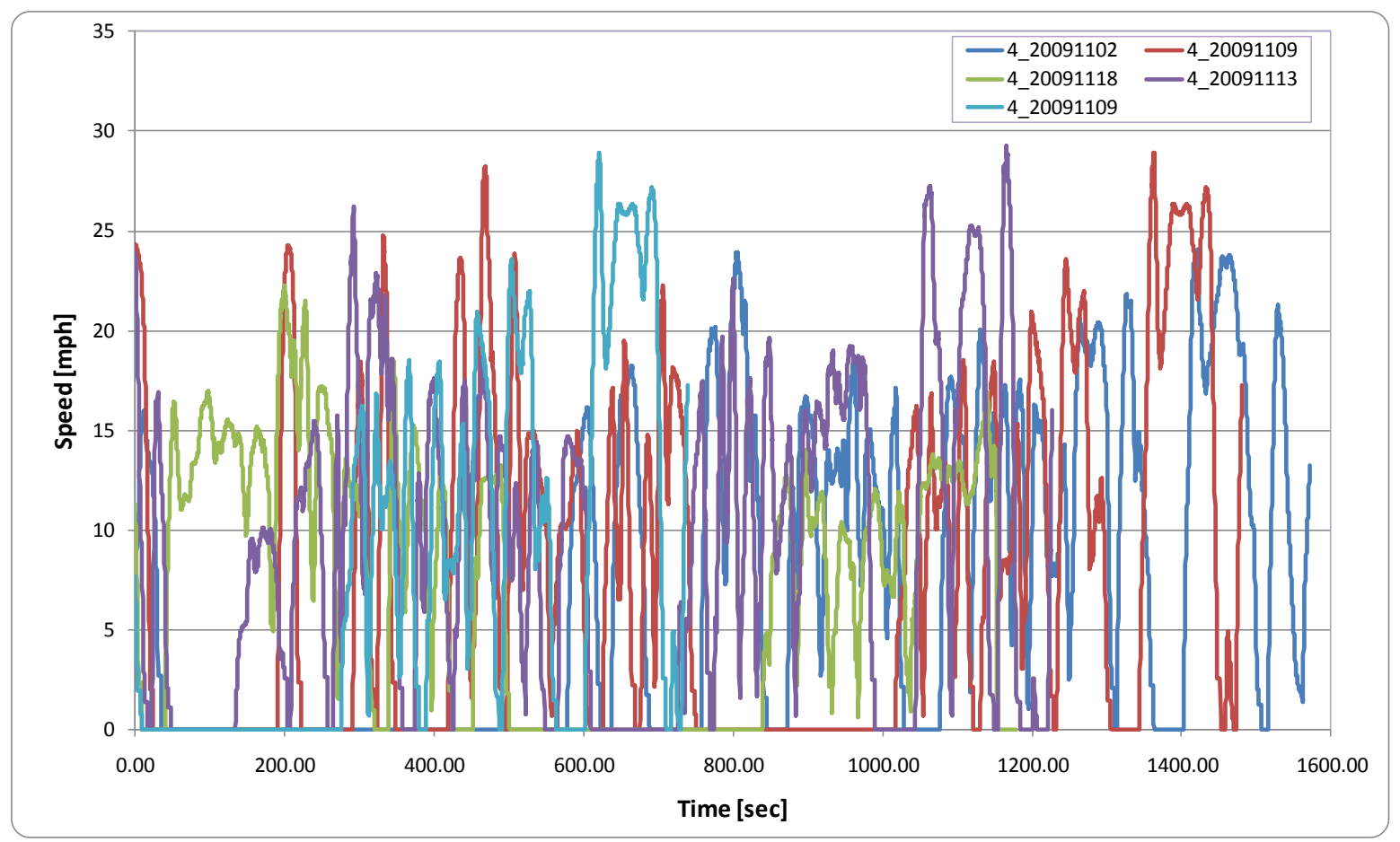

Figure 45. Bottom Five Maximum Average Speed Transit Bus Surface Street Duty Cycles 


\subsection{SAFETY SENSOR DATA}

Beyond the standard duty cycle data collection system required for the MTDC project, additional sensors were installed on three test vehicles (Class-7 combination delivery vehicles) to collect several safetyrelated signals of interest to FMCSA. These additional sensors were provided through a partnership with FMCSA. The real-time brake stroke, tire pressure, and weight information obtained from these sensors is expected to make possible a number of safety-related analyses such as determining the frequency and severity of braking events and tracking tire pressure changes over time. Because these signals are posted to the vehicle's data bus, they also have the potential to be read by on-board telematics solutions for inclusion in other FMCSA efforts such as the Wireless Roadside Inspection (WRI) Safety Data Message Set (SDMS). Due to the "drop and hook" operation of the host carrier, instrumentation was not installed on any of the carrier's trailers. Although ORNL's primary task was the collection of this data rather than the performance of any analyses, a preliminary analysis of the safety sensor data was performed to determine how the sensors had functioned during the data collection period and to explore the types of information available from this expanded data set.

\subsubsection{Findings from Safety Sensors}

Each sensor provided useful information related to the duty cycle of the vehicle and/or the effectiveness of the sensor. Unless otherwise specified, the following results are based on analysis of the data compiled from all three test vehicles. This data represents approximately 45,000 miles of local delivery duty cycle data.

\subsubsection{Electronic Brake Stroke Monitoring System}

Four brake stroke sensors were installed in each vehicle, one at each wheel-end of the tractor. The in-cab unit provided the driver audible alerts of out-of-stroke conditions. In addition, a brake system pressure transducer provided information about braking events in the duty cycle data. The brake stroke system provided real-time status codes for each wheel position. A histogram of these codes is shown in Figure 46.

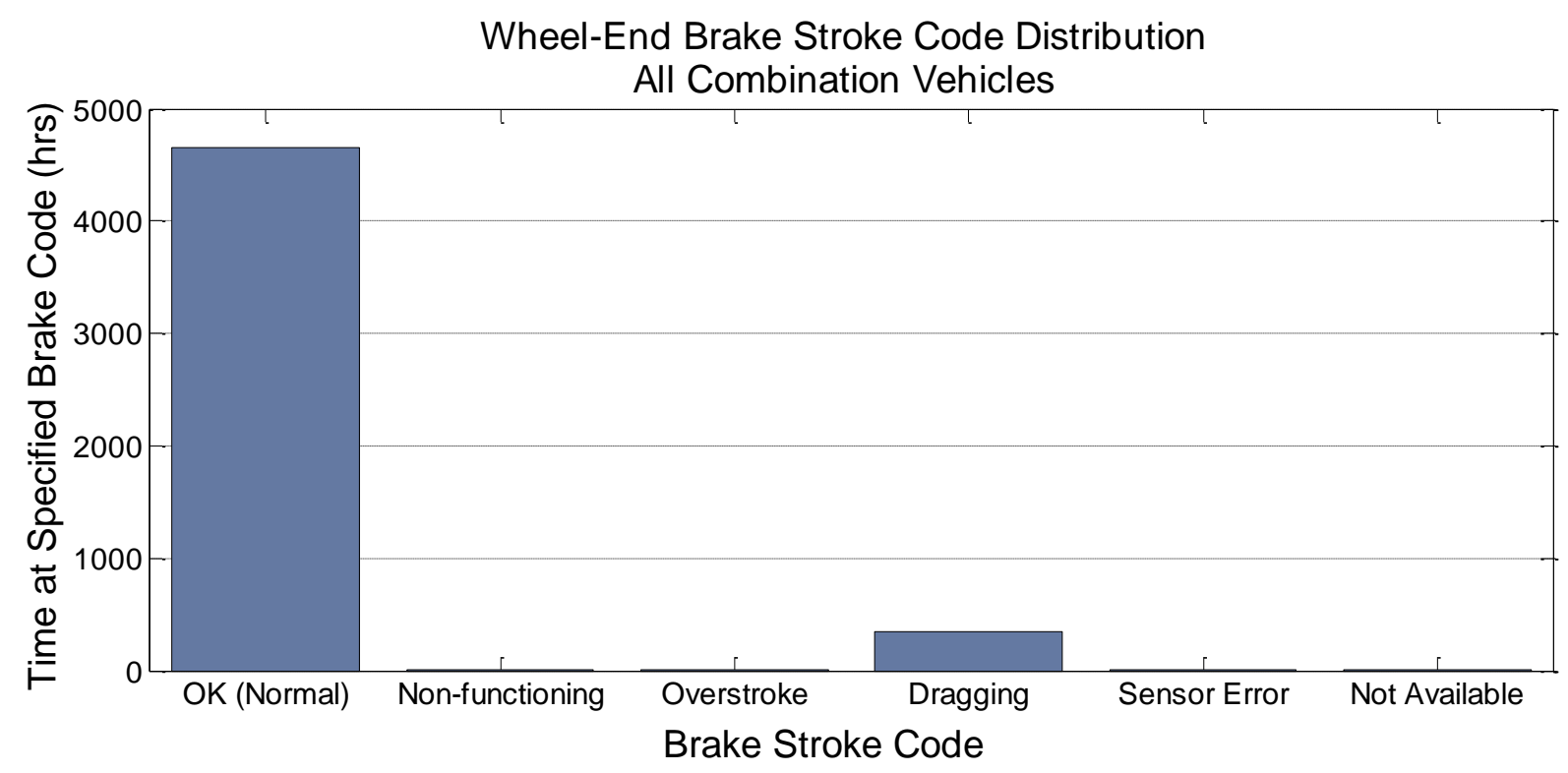

Figure 46. Distribution of Brake Stroke Status Codes 
From the time the systems were first installed, one sensor continually output a dragging brake code. The manufacturer was not able to correct this error, and the team decided to proceed with the FOT with plans to discard data from this sensor. This errant reading yielded to an overall error of $6 \%$ (for all three vehicles).

The brake system pressure transducer provided information about the overall brake application pressure, identifying braking events in the duty cycle data. The chart shown in Figure 47 illustrates the low percentage of time the test vehicles were in engaged in braking events.

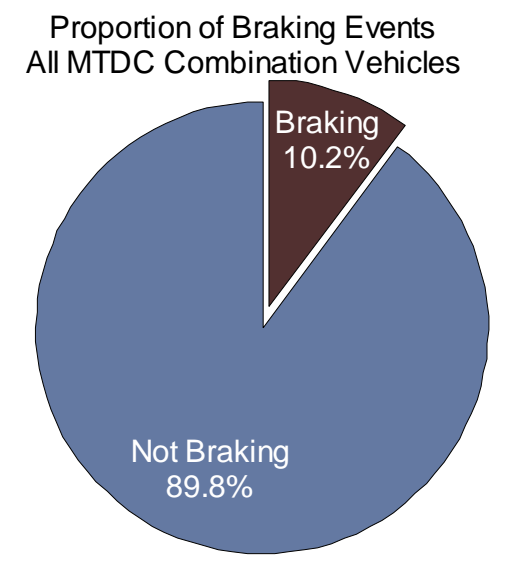

Figure 47. Proportion of Braking Events in MTDC Combination Vehicle Duty Cycles

Of interest to FMCSA is the frequency of hard braking events, characterized by higher brake application pressures. The data collected in this FOT indicates that lower-pressure braking events were by far more common than hard braking events. From the distribution of brake application pressure for this FOT (Figure 48), it is evident that the brakes are nearly always applied at pressures below 30 psi.

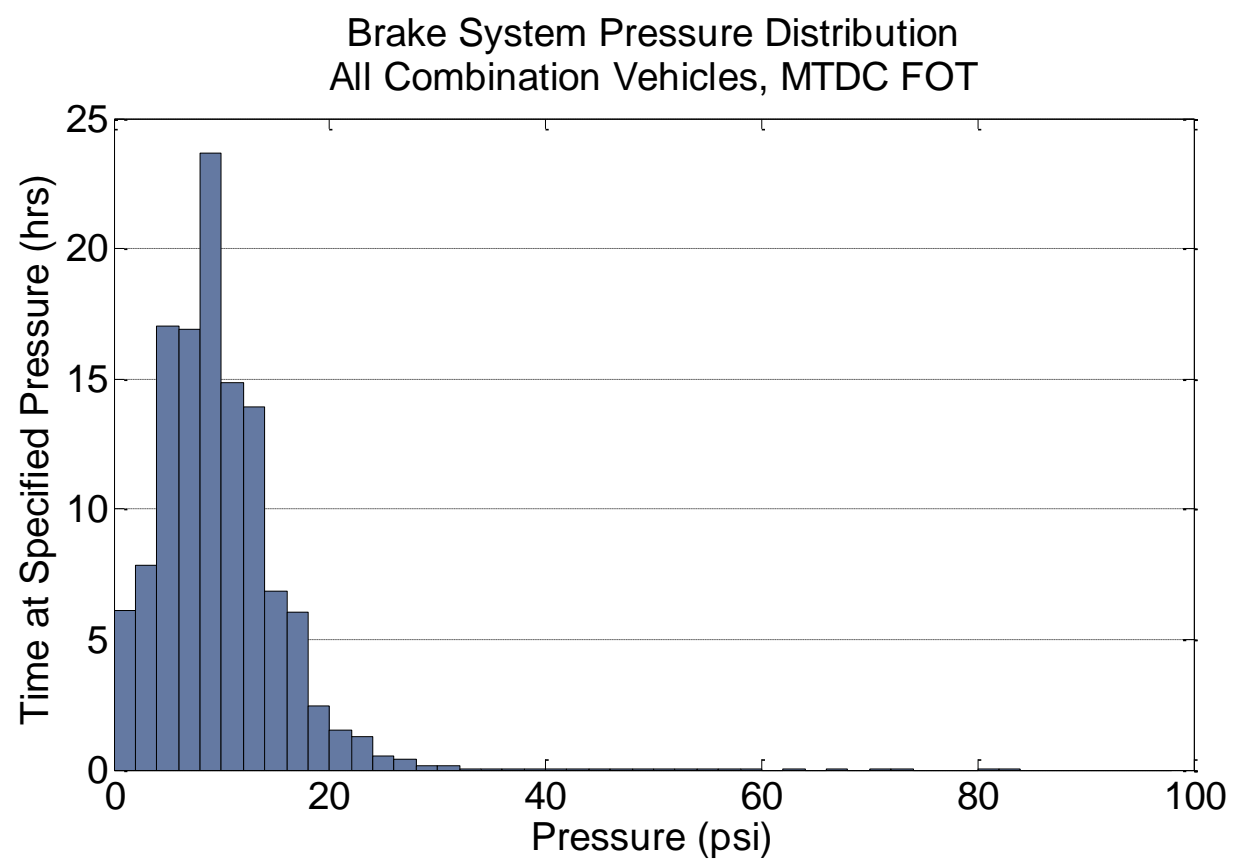

Figure 48. Distribution of Brake Application Pressure for MTDC Combination Vehicles 
The pressure signal provided by this sensor was reliable, with no detected problems. The time history of this signal, taken in conjunction with the vehicle speed, can be used to examine specific braking events. Figure 49 shows a 2,000-second section of duty cycle data from one of the test vehicles in which there were several braking events. In this figure, it is apparent that significant decreases in vehicle speed (green trace) are caused by braking events, indicated by increases in brake application pressure (blue trace).

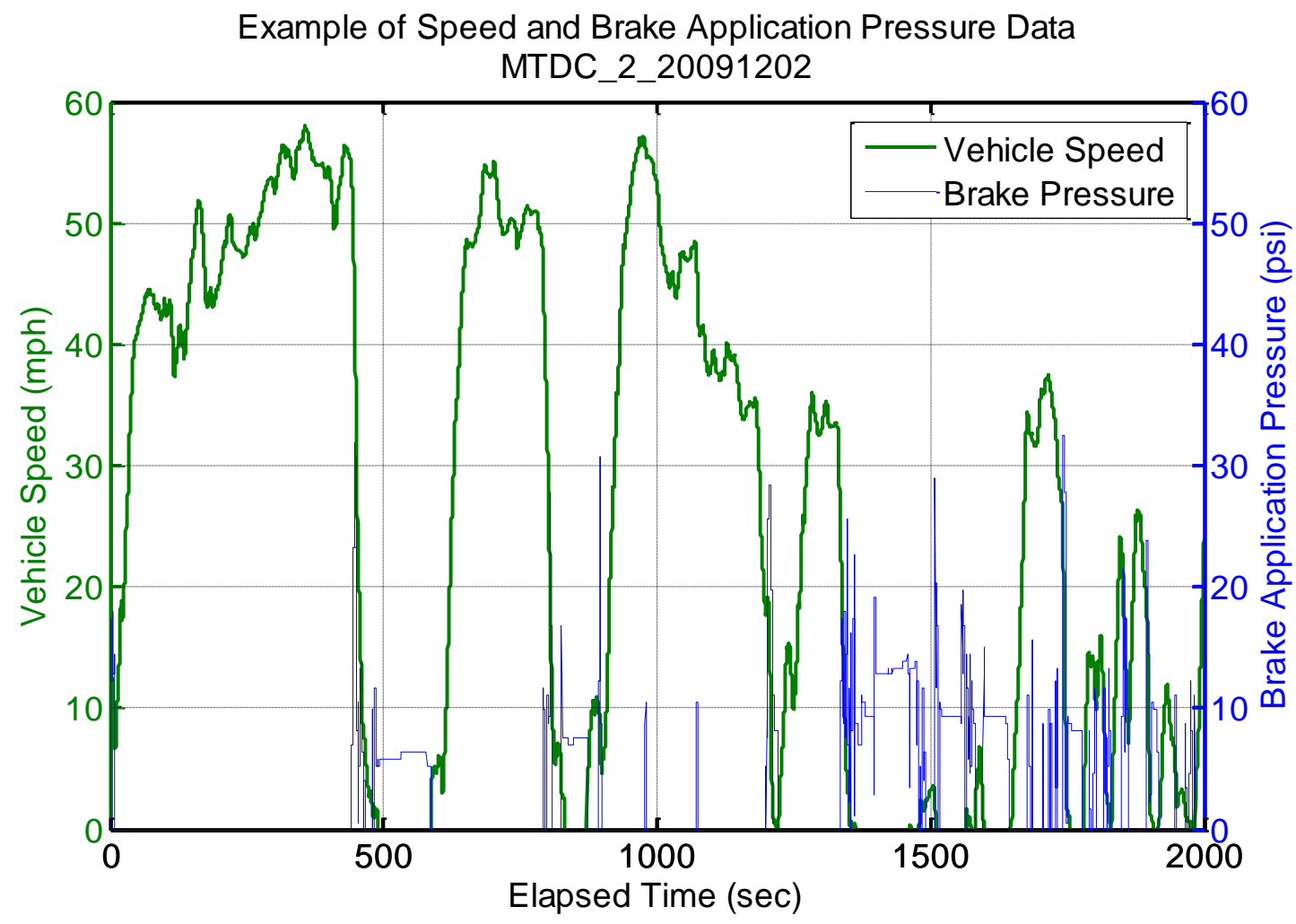

Figure 49. Sample Time History of Speed and Brake Application Pressure

\subsubsection{Tire Pressure Monitoring System}

The tire pressure monitoring system (TPMS) included a pressure/temperature sensor for each tractor tire for a total of 6 sensors per vehicle. An in-cab display provided the driver with the temperature and pressure for each tire and an audible alert for out-of-range values indicative of a problem (e.g., a tire with low tire pressure). Because the partnering fleet checked the tire pressures weekly, no out-of-range values were expected to be encountered except as a result of tire damage since the last air pressure check. The distribution of tire pressure for one wheel-end of one of the test vehicles is shown in Figure 50. This histogram indicates sensor values were in-range the majority of the time, although out-of-range values did occur and several flags were set (Figure 51). 


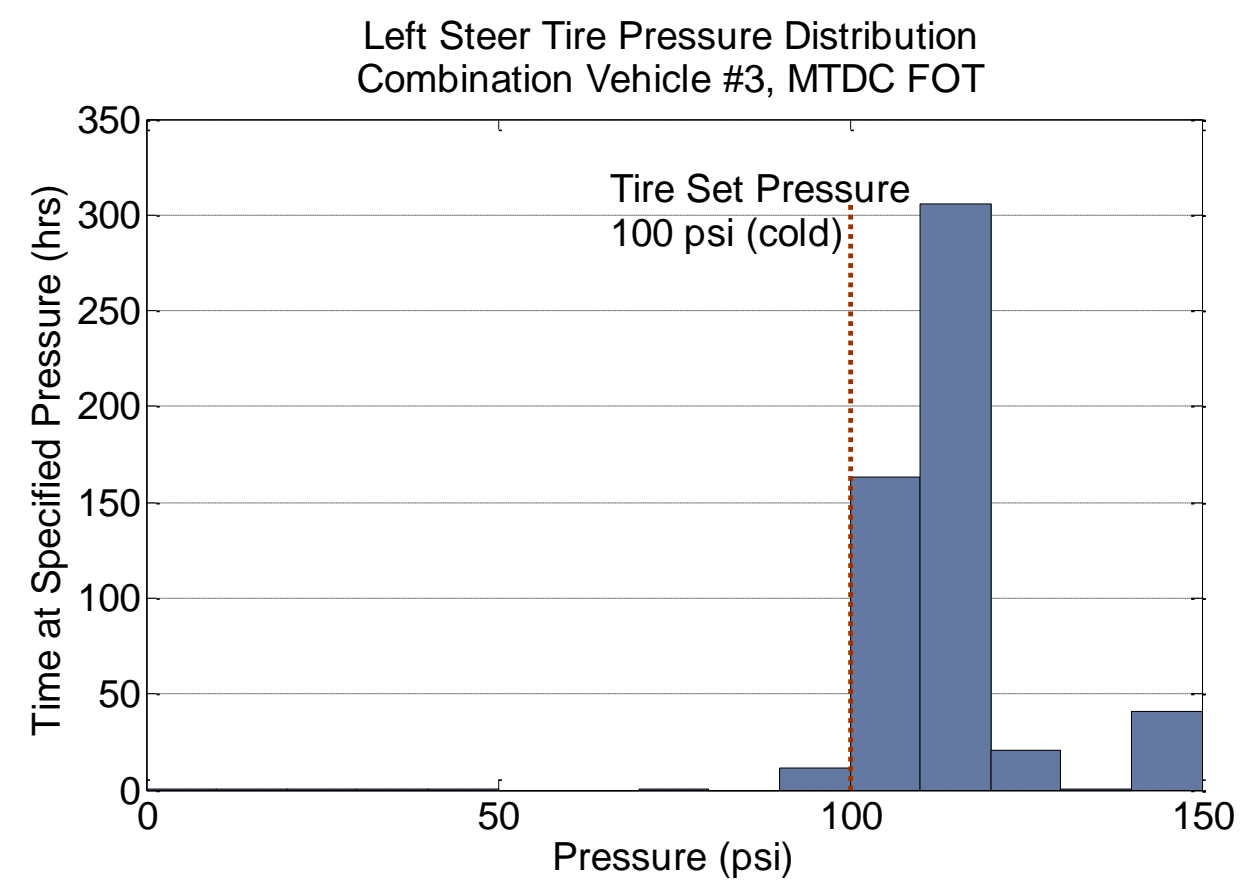

Figure 50. Tire Pressure Distribution

When a tire pressure reading was outside the acceptable range as defined in Table 19, the TPMS generated a corresponding error code for that tire (Figure 51). The driver investigated the error codes generated but did not identify any problems during the course of the FOT. During this interval, the error rate for the tire pressure monitoring systems' alert codes represented more than $20 \%$ of the data collected.

Table 19. Pressure Ranges for TPMS Status Codes

\begin{tabular}{|l|l|}
\hline Pressure Status Code & $\begin{array}{c}\text { Corresponding } \\
\text { Range }\end{array}$ \\
\hline Extreme Over Pressure & Over 145 psi \\
\hline Over Pressure & $125-145 \mathrm{psi}$ \\
\hline No Warning & $75-125 \mathrm{psi}$ \\
\hline Under Pressure & $50-75 \mathrm{psi}$ \\
\hline Extreme Under Pressure & Below 50 psi \\
\hline
\end{tabular}


Tire Pressure Code Distribution

All Combination Vehicles

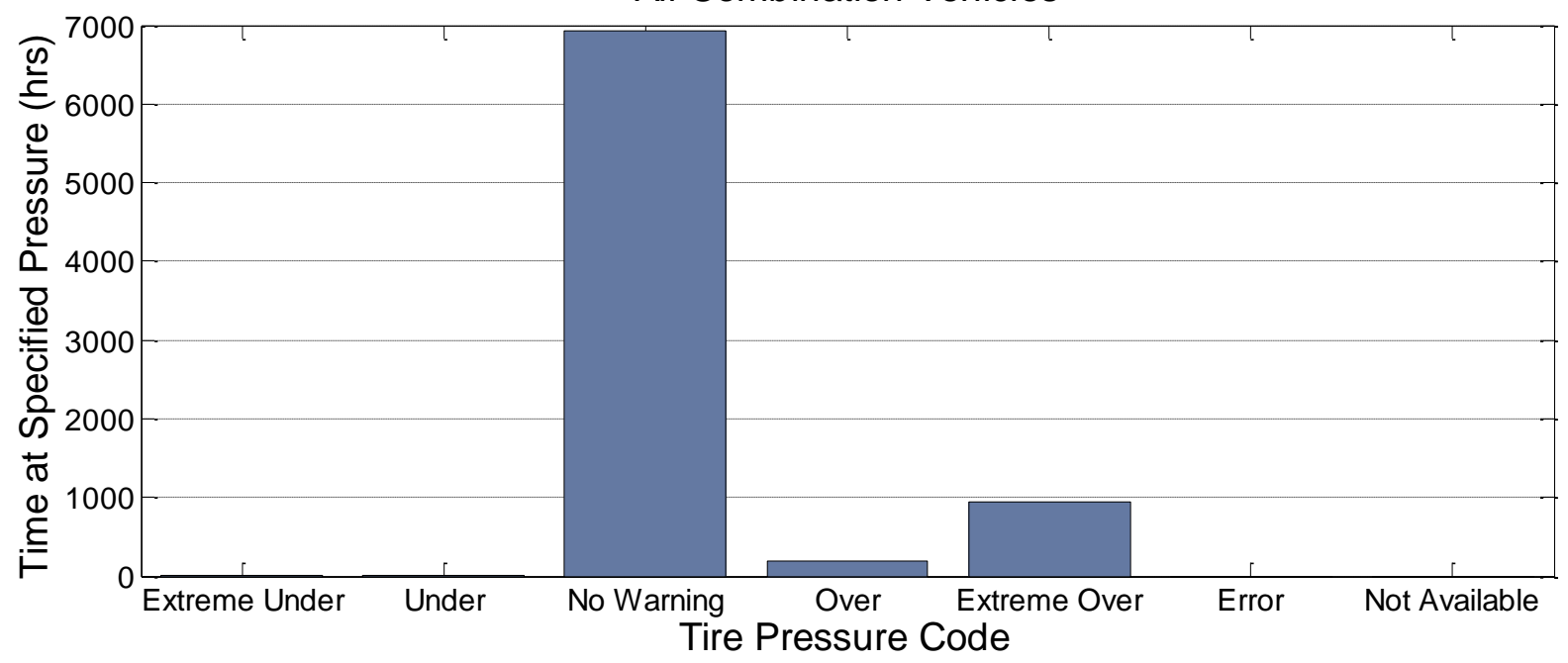

Figure 51. Distribution of Tire Pressure Threshold Detection Codes

Each tire pressure sensor also had temperature-reading capability. A histogram of this data is shown in Figure 52.

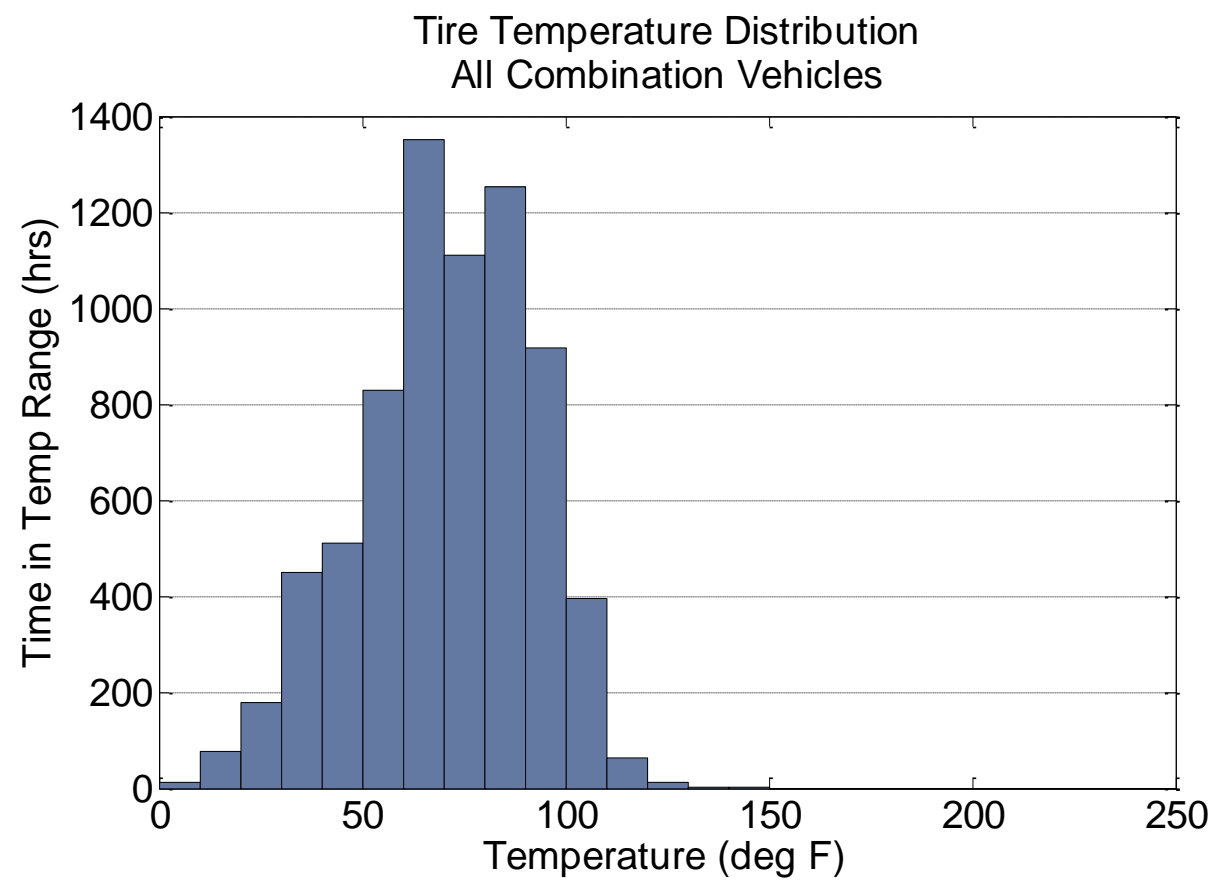

Figure 52. Tire Temperature Distribution

Researchers, drivers, and maintenance technicians reported a number of additional problems associated with the TPMS throughout the FOT, such as nonfunctioning sensors, confusion of wheel-positions reported, significant temperature differences between wheel positions at a given time, and discrepancies between the in-cab display and the values which were reported on the data bus. 


\subsubsection{Vehicle Self-Weighing System}

The self-weighing system reported drive and steer axle weights based on sensors in the vehicle's air suspension system. Although readings were taken continuously, the system was not designed for accuracy while the vehicle was moving; ORNL has a preliminary algorithm to infer actual weights from reported values on the instrumented axles (section 3.5). The reported weights are in the range expected for class-7 vehicles (up to 12,000 and 20,000 lbs respectively) for the steer and drive axles (Figure 53 and Figure 54), with very few out-of-range values reported. As expected, the drive axle weight readings were fairly constant.

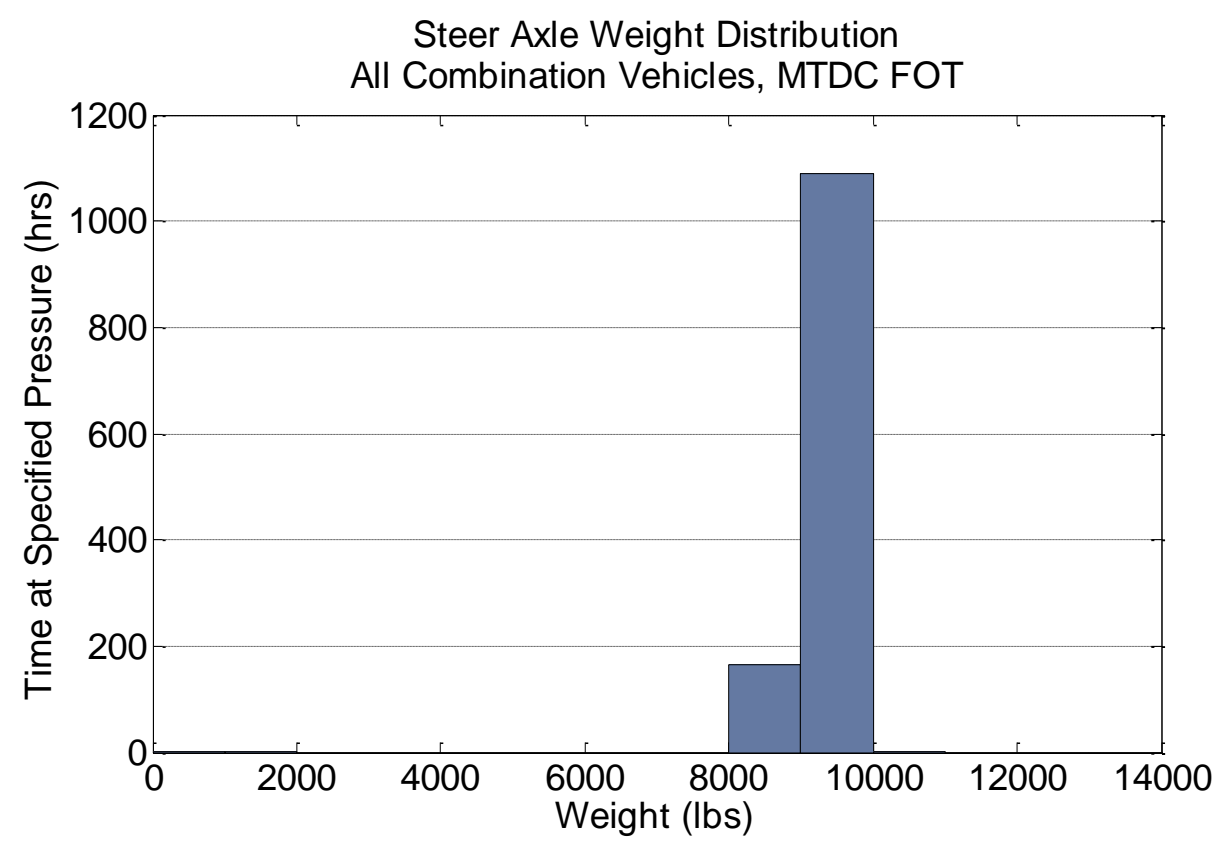

Figure 53. Distribution of Steer Axle Weights

Although the fact that this system was not designed for accuracy while the vehicle was moving, unloaded and loaded approximate weights can be identified in the drive axle group weight distribution shown in Figure 54. 
Drive Axle Weight Distribution

All Combination Vehicles, MTDC FOT

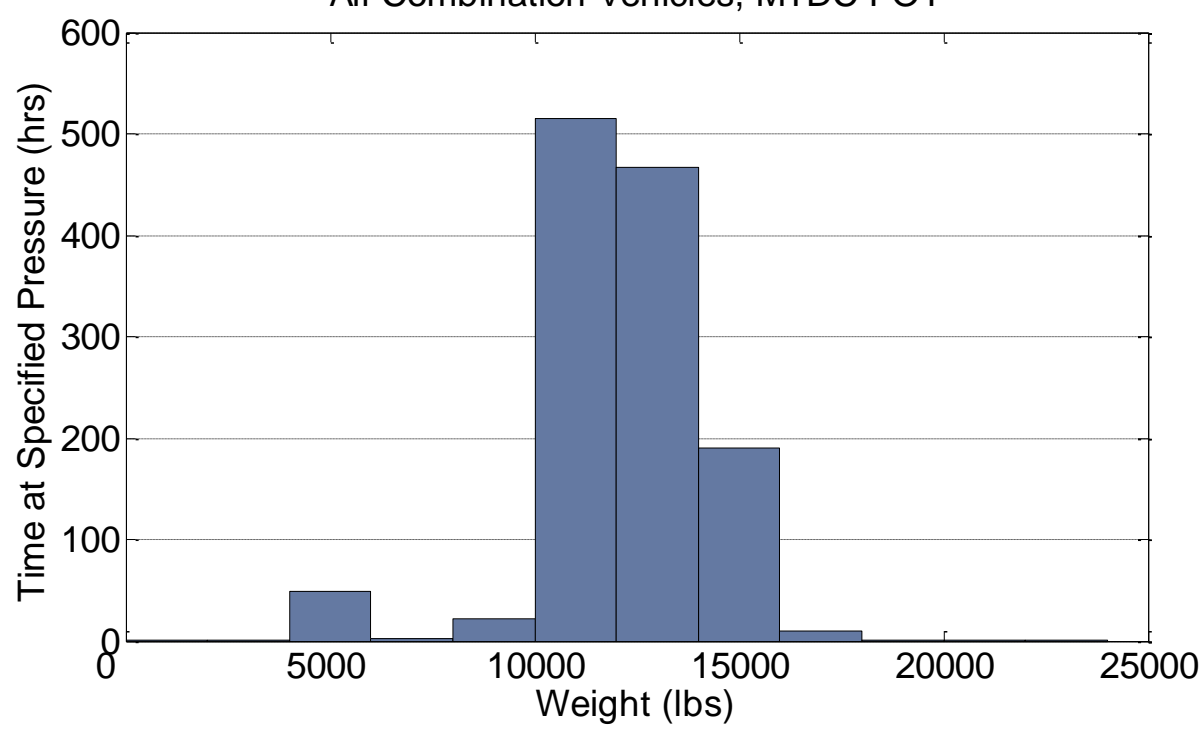

Figure 54. Distribution of Drive Axle Weights

\subsubsection{Implications for Research}

\subsubsection{Post-Processed vs. Real-Time Data}

Due to the number of errors present in the brake stroke and tire pressure monitoring systems, this data requires additional post-processing to filter out the errors before further analysis. This is not expected to cause difficulty for non-real-time uses such as determining the number and severity of braking events in a given duty cycle.

Although the vehicle self-weighing system was not designed to report accurate weights for a vehicle in motion, streaming data from this system was sufficiently accurate to estimate loading (e.g., unladen, medium load, fully laden). After completion of the weight prediction algorithm, post-processing could be used to achieve more resolution from these signals.

Based on the errors in the tire pressure monitoring and brake stroke systems, post-processing based on an interval of data is required before the data can be used. Real-time use of data from the self-weighing system is suitable only for general estimates of the weight (e.g., as an indicator that the vehicle is lightly laden). This limitation does not preclude the near-real-time uses involving on-board processing.

\subsubsection{Specific Applications of Data}

The positive results obtained from the brake application pressure sensors have led to follow-on research in FY2011 for ORNL in FMCSA's Real-Time Dynamic Brake Assessment project. This effort will build on the sensor work done in the Part 1 MTDC FOT, using a modified brake application pressure sensor on one of the instrumented vehicles to further study low-pressure braking events. This proof-of-concept testing will examine the feasibility of an on-board brake assessment tool based on real-world driving data, such the braking event shown in Figure 55. 


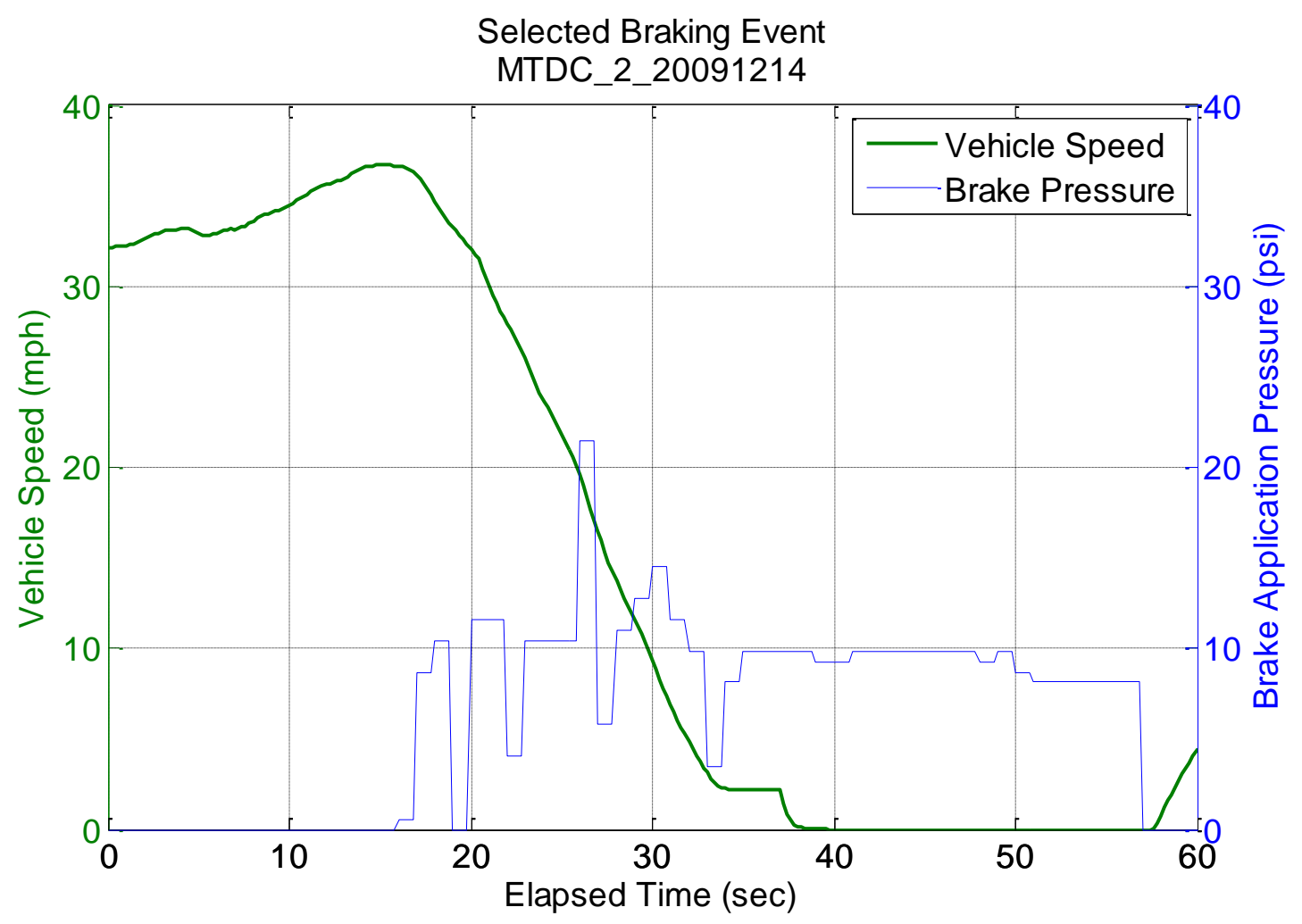

Figure 55. Selected Braking Event

Because all the safety sensors studied posted data to the vehicle's data bus, and therefore have the potential to be read by any on-board system, one potential use of the data involves inclusion in the SDMS for FMCSA's WRI program. While these sensors can provide valuable advisory information, it is recommended that at this stage such safety sensors not be used to flag WRI test vehicles for further inspection for several reasons:

- "False alarms" and sensor errors were consistently present in one of the brake stroke monitoring systems.

- There were a number of errors and discrepancies between the in-cab and data bus reported readings for the tire pressure monitoring system data.

- The self-weighing system for static weights was designed for static weighing on level ground with the brake released, not over-the-road, instantaneous weight measurements. 


\section{LESSONS LEARNED}

The lessons learned in the Part 1 MTDC FOT data collection and analysis period were varied. Some lessons learned involved successful improvements over the previous HTDC work. Other lessons learned were immediately incorporated into the testing protocols being employed at the time, and allowed for more productive and efficient research and data collection. An additional category of lessons learned were of a nature that did not allow for more immediate or short-term adaption, but could be addressed in the Part 2 MTDC FOT or other future research.

\subsection{IMPROVEMENTS FROM HTDC}

Several changes were made to the data collection methodology based on lessons learned from previous duty cycle work such as the HTDC Project. Some improvements or lessons learned were not under the direct control of the project team but were a result of the nature of medium-truck operations for the vocations studied.

\subsubsection{Modifications to Data Acquisition System}

A key improvement to the DASs for the Part 1 MTDC FOT was the inclusion of a cellular modem to facilitate remote data transfers. However, upon implementation of this methodology, a number of additional benefits became apparent. Not only did this additional equipment make possible the automated retrieval of data from the test vehicles (greatly reducing the need for research staff to be involved in the day-to-day FOT operations), but it allowed the retrieval to be done more frequently. Because the data retrieval software was integrated with an automated error-checking routine, this led to much quicker error detection and response.

Because of the problems involved in the repurposing of weather stations for vehicle use as was done in the HTDC work, no weather station was installed on the test vehicles. Instead, ORNL worked with technicians at the partners' respective shops to make a connection to the vehicle's wipers. This allowed basic weather information regarding precipitation to be collected without the installation of any additional equipment. In addition, temperature measurements were read from the test vehicle's data bus, where available.

To streamline the installation, the DAS wiring was improved. Bulkhead connectors were installed in the DAS's case to make possible the separate installation of the cabling. The mating connectors were incorporated into pre-made cabling which was given to the partners for installation by their technicians.

\subsubsection{Coordination with Fleet Partners}

One problem occasionally encountered in the HTDC FOT was the limited availability of the test vehicles for data download and correction of minor sensor problems. Medium truck operations typically run on a more predictable schedule, making visits to correct equipment more easily scheduled. In addition, ORNL was able to interface with a main point of contact in each of the partners' shops to check on equipment and coordinate visits. This led to reduced down-time and loss of data due to easily-corrected sensor problems. In addition, ORNL partnered with fleets in the local area rather than those more than an hour away, as was the case in the HTDC efforts; this resulted greatly reduced travel times when such visits were needed.

In the HTDC project, both tractors and trailers were instrumented with the Air-Weigh self-weighing unit. Although the fleet management indicated they would attempt to see that instrumented tractors and trailers 
were mated as often as possible, this proved impractical for their drop-and-hook operation.

Consequently, only a small percentage of the data collected involved an instrumented trailer. Because the delivery fleet partner used a drop-and-hook operation, a decision was made not to direct resources toward the instrumentation of any trailers due to the small amount of additional data that would be available in their operation.

As a result of the lessons learned from the HTDC, ORNL was able to work better with the fleet partners, not expecting any departure from their standard operating procedures. For example, ORNL discussed standard procedures with fleets prior to instrumentation to prevent damage to the GPS antennas due to pressure-washing. Certified weight information was obtained by coordinating planned trips to inspection stations to obtain weigh tickets for the weight estimation model. In addition, rather than attempting a long-term collection of fuel tickets for calibration of the fuel model (which proved unsuccessful in the HTDC efforts), a short-term study was performed with driver incentives for participation during the onemonth period. This methodology proved successful, providing the necessary data with high accuracy.

\subsection{PLANNING FOR PART 2 OF THE MTDC EFFORT}

Although the method of making vehicle connections was a significant improvement over that used in HTDC, the partner technicians frequently had to make several different connections to a few contact points to accommodate the additional sensors installed. As a result, wires would occasionally become disconnected and have to be replaced. To correct this problem, a single Data Acquisition System Interface Box (DASIB) will be constructed for each vehicle. This will allow a single connection to be made to each key point in the vehicle (data bus, power, etc.). Mating connectors will be made so that additional sensors can interface to the DASIB, which can be configured as necessary to accommodate different types of sensors.

Technical difficulties were encountered with the VBOX GPS unit, particularly with the combination vehicles. Due to the inability of the systems to maintain satellite lock for long periods of time, the units frequently had to be manually reset or "cold started," approximately once every few weeks. Because the channels were checked each time the data was uploaded, this problem was identified and corrected soon after the problem occurred, resulting in a minimal loss of data. Corrective actions included replacing the GPS antenna, ensuring that the units were not subjected to frequent power cycles and ultimately sending the units in for repair. When these failed to determine a cause of the frequent problem, the decision was made to explore other $5 \mathrm{~Hz}$ GPS solutions compatible with the eDAQ-lite. HBM, the manufacturer of the eDAQ-lite, now produces a layer with a built-in GPS of the needed resolution. This layer was tested with the DASs and found to be comparable to the VBOX; this layer will be installed in all of the DASs for the Part 2 MTDC FOT, and the VBOX units were removed from the DASs.

\subsection{ADDITIONAL LESSONS LEARNED}

During the Part 1 MTDC FOT, the majority of the equipment functioned as expected. Preliminary testing of each DAS allowed most of the problems to be discovered and resolved before initiation of the FOT.

The problems which were not foreseen during the early stages of the testing involved the long-term nature of the project which could not be practically simulated prior to the start of the FOT.

In order for the data to be retrieved remotely, the DAS had to be powered up; for this remote upload to take place on a regular basis, the DAS had to be left on at all times. However, this resulted in frequent problems with draining of the vehicle's battery. To correct this problem, digital timers were installed to "wake" the system at scheduled data upload times during the night and also to ensure the systems were on at a scheduled time during the day in the event of the occasional problem with the automated process. 
This solution proved successful in both eliminating the battery problems for the fleet partner and making the vehicles available for remote access.

Toward the beginning of the Part 1 MTDC FOT, the combination vehicles were experiencing problems due to an unavoidable drop in voltage when the engine started. Following the recommendation of the fleet partner's shop manager, a capacitor was installed to provide a more constant voltage during the brief startup period. This solution proved successful, and although this problem had not yet been encountered on all the vehicles, capacitors were also installed on the remainder of the test vehicles to avoid this problem.

The time required to install the safety sensors was longer than expected, inconveniencing the partners and impacting fleet operations. Although the partners were willing to accommodate by continuing to allow the sensor technicians to use their shop and rearrange their delivery schedules, future installations should be better understood to minimize the impact to the partners.

The particular tire pressure monitoring system selected for this program proved problematic in some instances. Personnel from the partnering fleet reported false alarms and the occasional unresponsive sensor. Additionally, the values reported on the data bus were found to not always agree with the in-cab display.

The complexity of the DAS meant that equipment issues typically resulted in slightly long delays as these problems were corrected in accordance with standard procedures characteristic of a national lab (working through telecommunications and/or procurement departments). Although the budget for this project did not support the purchase of a complete spare system in the event of failures, the construction of such a spare may prove cost-effective for future projects where unanticipated delays of 1-6 weeks are unacceptable. 


\section{SUMMARY OF RESULTS/CONCLUSIONS}

In this part of the MTDC project, three Class-7 tractors from the H.T. Hackney fleet and three transit buses from the KAT were instrumented and over 70 channels of data were collected for over a one-year period of time at a rate of $5 \mathrm{~Hz}$. The data gathered in this project included information such as instantaneous fuel rate, engine speed, gear ratio, vehicle speed, and other information read from the vehicle's data bus; spatial information (latitude, longitude, altitude) acquired from a GPS device; and instantaneous tractor (for the combination trucks) and bus weight obtained from devices mounted on the six participating vehicles. Some of the vehicles were also mounted with safety sensors providing brake and tire information in partnership with FMCSA, yielding valuable information to support related research efforts. During the one-year data collection period, the six participating vehicles logged over 95,000 miles (45,400 for the combination trucks and 49,400 for the transit buses) and consumed over 17,000 gallons of fuel (6,000 for the combination trucks and 11,300 for the transit buses), while conducting business in the East Tennessee area. Use of the test vehicles, and costs related to fuel, driver's time, insurance, and the use of fleet partner's facilities for vehicle instrumentation and DAS maintenance were provided gratis to this project. In addition, the data gathered from the FMCSA brake and tire systems are available to ORNL and DOE at no additional cost. These partnerships represent a significant cost savings to DOE, and made possible the collection of real-world-based operational data for medium trucks.

For the combination trucks, the largest proportion of idling time (61\%) and fuel consumed (50\%) while idling correspond to idling intervals that lasted between 0 and 5 minutes (i.e., traffic congestion and delay at traffic signals). This is followed by intervals of 5 to 10 minutes of idling time ( $25 \%$ of idling time and $25 \%$ total fuel consumed while idling), and by the 15 to 60 minute time interval. The latter involves mostly idling while stopping for a delivery. The 180 to 240 minute interval is proportionally low in terms of idling time (1.5\%) but ranks third (12\%) in terms of fuel consumed while idling. The transit buses also spent most of their idling time (31\%) in congestion and bus dwelling stops ( 0 to 5 minute idling interval) which also consumes the largest proportion of fuel spent while idling. However, as opposed to the combination trucks, the transit buses spent $26 \%$ of their idling time in intervals that are longer than 4 hours, also consuming about $26 \%$ of the fuel spent while idling. These large idling times were observed mostly at the parking lot while the vehicles were waiting to start a trip and are currently being addressed by KAT to reduce fuel usage.

Prior to using the fuel data available from the data bus, a short-term study was performed to ensure that the source of this information provided an accurate representation of fuel usage. After that study was conducted with the results showing a high accuracy in the fuel information provided by the vehicle data bus, the data collected in the project was used to compute the overall fuel efficiencies; that is, the fuel efficiencies computed by taking into consideration idling times, as well as the moving fuel efficiencies (i.e., fuel consumed only when the vehicle was moving) of combination trucks and transit buses were computed. For the transit buses the percentage difference between overall and moving FEs is much higher (between 10 and 20 times higher) than that of the combination trucks. Some of this difference can be attributed to differences in duty cycles and drivetrains, but most is due to idling. While the total miles traveled by the two sets of vehicles during the year-long project is almost the same, just less than $10 \%$ larger for the transit buses than for the combination trucks, the former consumed almost 20 times more fuel than the latter while idling. Using the moving FE information, it is possible to see the FE of the two types of vehicles is very different, being almost $40 \%$ higher for the combination trucks than for the transit buses.

One very important variable affecting the fuel efficiency of any vehicle is its payload level. Based on vehicle weight data collected at commercial scales (i.e., Inspection Stations in Knox County, Tennessee), 
ORNL developed a model that predicted the trailer weight based on the tractor weight provided by the Air-Weigh device. This model was used to assign trailer weight to each one of the records in the database of information collected in this project. For the transit buses, the deployed Air-Weigh devices provided weight information for the entire vehicle, and therefore it was not necessary to develop a payload estimation model in this case.

For the combination trucks, the vehicle weight was divided into four categories: 1) Tractor with Empty Trailer, 2) Light Load (total vehicle weight between 27,000 lbs and 31,999 lbs); 3) Medium Load (total vehicle weight between 32,000 lbs and 37,999 lbs); and 4) Heavy Load (total vehicle weight above $38,000 \mathrm{lbs})$. In the case of the transit buses, the vehicle weight was divided into three categories: 1) Empty Bus (vehicle weight less than 22,500 lbs), 2) Light Fare (total vehicle weight between 22,500 lbs and 25,499 lbs); and 3) Heavy Fare (total vehicle weight above 25,500 lbs).

On average, the combination trucks weighted between $27,700 \mathrm{lbs}$ to 29,000 lbs and the buses between 23,000 lbs and 23,800 lbs. Very few trips with a tractor and empty trailer were observed. Most of the trips for vehicle $2(98 \%)$ were at a light or medium load, while for vehicle 3, almost $70 \%$ of the trips were at the medium and high level loads. Transit Buses 2 and 3 (i.e., vehicles 5 and 6) operated almost 90\% of the time with a light fare. Transit Bus 3 also had the largest average weight, indicating that this was the vehicle that carried the largest number of passengers (on average). Transit Bus 1 (i.e., vehicle 4), on the other hand, spent almost $30 \%$ of the time at an empty level. This was mostly due to idling at the parking garage. Transit Bus 1 had the lowest overall FE out of the three buses.

To generate the distribution of fuel efficiency under the payload levels described above, 10-mile segments were considered for which the fuel efficiency was computed and counted as one observation. Overall, and as expected, the FE decreases as the payload increases for the combination trucks. However, because the payload categories are very narrow and low (those combination trucks never made any trips that were above 42,000 lbs, far below the legal weight limit for Class-7 trucks), the variation of the FE is not significant. In the case of the Transit Buses, the relationship between FE and vehicle weight is not as it would be predicted (i.e., decreasing FE with increasing payload). This is due to several factors. First, when the vehicle is empty (lowest payload) it spends a considerable amount time idling. This results in very low FEs for this load-level category. At the other end of the spectrum, the highway routes (express routes) are the ones that carry the largest number of passengers. The buses on these routes spent very little dwelling time (there are fewer bus stops) and also encounter the least number of traffic lights. This results in FEs that are higher than regular surface street routes (light fare) that have many more stops (bus stops and traffic signals).

The data collected in this project was also used to investigate the variability that may exist in duty cycles that are generated by the same vocation and that follow the same route. In order to do this, two sets of duty cycles collected from the data gathered by the transit buses were identified. The first set focused on freeway duty cycles. A $19 \mathrm{~km}$ segment of Westbound I-40 in Knoxville, Tennessee was selected and the corresponding duty cycles extracted from the database. The duration of the 74 highway duty cycles ranged from 10.5 minutes to 27.6 minutes with an average duration of 12.6 minutes and a standard deviation of 2.71 minutes.

In order to analyze surface-street transit duty cycles, a $4.9 \mathrm{~km}$ loop in downtown Knoxville, Tennessee was selected and the corresponding duty cycles extracted from the database. Thirty-eight surface street duty cycles with the characteristics described above were selected. The duration of the duty cycles ranged from 10.9 minutes to 22.8 minutes with an average duration of 18.4 minutes and a standard deviation of 3.29 minutes. 
This variability of the duty cycles within a given category (i.e., freeway or surface street duty cycles) could be measured by the ratio of the standard deviation of the distribution of duty cycle duration over the mean of that distribution. For the 74 duty cycles this ratio is 0.214 (i.e., 2.71 minutes/12.7 minutes). For the five top and five bottom duty cycles - ranked using the average speed of the duty cycle- the variability was computed at 0.06 and 0.357 , respectively. When they were combined (i.e., in ten duty cycles) the variability increased to 0.402 . In the same way, the 38 surface street duty cycles had a ratio equal to 0.179 (i.e., 3.29 minutes/18.4 minutes). For the five top and five bottom duty cycles, the variability was computed at 0.134 and 0.262 , respectively. When they were combined (i.e., in ten duty cycles) the variability increased to 0.308 . Using this measure, the highway duty cycles presented a higher variability than the surface street duty cycles. 


\section{REMAINING MTDC TASKS}

Due to the number of vocations represented in medium trucks, the MTDC data collection effort is divided into two parts. In addition to a second phase of data collection, a number of related tasks will be completed in the Part 2 MTDC efforts.

\subsection{DATA ACQUISITION SYSTEM MODIFICATIONS}

In preparation for the Part 2 MTDC FOT, the DAS will be updated to include a different GPS sensor and to incorporate a DASIB, in accordance with the lessons learned. In addition, the maker of the eDAQ-lite and its associated software has transitioned to a new file format which provides additional flexibility in data retrieval. The DASs and ORNL-developed data retrieval and processing software will be transitioned to this new "SIE" (file descriptor assigned by HBM/SoMat) format to take advantage of these additional features beginning with the Part 2 MTDC effort.

\subsection{PART 2 MTDC FOT AND DATA ANALYSIS}

The Part 2 MTDC FOT will continue the medium truck data collection effort with a focus on the utility and towing/recovery vocations. The 12 months of data collected during this second FOT will be analyzed to examine the effect of PTO on energy efficiency; in addition, similar data analyses to the ones conducted in this phase of the project will be performed. These will include studies on the effect of vehicle payload, as well as the effects of grade and speed on fuel efficiency. A study on duty cycle variability of the new two vocations and compared to the variability observed in transit bus and combination truck duty cycles.

\subsection{DUTY CYCLE GENERATION TOOL}

The DCGenT is an ORNL/CTA-developed software tool which allows the user to generate a synthetic but characteristic duty cycle from a set of real-world duty cycle segments. The synthetic duty cycle produced will have similar speed, acceleration, and idling characteristics as the original segments but with the overall duration determined by the user. The DCGenT will make use of a custom data selection tool to allow the user to select the criteria for the segments of source data. This allows the user to generate and analyze duty cycles for various combinations of driving conditions such as load, weather, grade, and congestion.

The DCGenT tasks to be completed during Part 2 of the MTDC effort include improvements to the tool as well as an expansion of the tool to include the ability to read additional data sets. The tool will be migrated from the original LabView application to a more user-friendly MATLAB-based tool with an improved user interface. The data collected during the MTDC FOT will be added to the existing database, making possible analysis of local delivery and urban transit data in addition to the heavy-truck long-haul data collected in the HTDC effort. These changes will be implemented in the March 2011 version of the software. Following the completion of the Part 2 MTDC FOT, data from utility and towing/recovery vehicles will also be included in the tool. A user's guide will also be created, providing an explanation of parameters and options, along with several examples of typical uses of the DCGenT.

\subsection{FINAL REPORT}

A final report will be prepared which describes the full MTDC program (i.e., Parts 1 and 2) including background information, partnerships, and data collection methodology. The test vehicles used in each part of the FOT will be described, and analysis of the collected data will be performed to obtain general 
statistics about each vocation with a focus on fuel efficiency. The report will also include key information such as lessons learned and recommended future research. 


\begin{tabular}{|c|c|c|c|}
\hline \multicolumn{4}{|c|}{ MTDC Interim Report Revision Log } \\
\hline \begin{tabular}{c} 
Number \\
\hline 0
\end{tabular} & 0 & Description of Change & $\begin{array}{c}\text { Change Effective } \\
\text { Date }\end{array}$ \\
\hline 1 & 0.1 & Initial outline & 3 Jun 2010 \\
\hline 2 & 0.2 & Added general/intro material & 17 Aug 2010 \\
\hline 3 & 0.3 & Converted to .docx & 20 Sep 2010 \\
\hline 4 & 0.4 & Additional sections of text & 21 Sep 2010 \\
\hline 5 & 1.0 & Completed draft & 4 Oct 2010 \\
\hline 6 & 1.1 & Comments from ags & 6 Oct 2010 \\
\hline 7 & 1.2 & Comments from gjc & 25 Oct 2010 \\
\hline 8 & 1.3 & Addressed comments, minor edits & 2 Nov 2010 \\
\hline 9 & 1.4 & Comments from hek & 10 Nov 2010 \\
\hline 10 & 1.5 & Addressed comments, minor edits & 19 Nov 2010 \\
\hline 11 & 2.0 & Revision complete & 19 Dec 2010 \\
\hline 12 & 2.1 & Second set of comments from hek & 3 Jan 2010 \\
\hline 12 & 2.2 & Final draft & 5 Jan 2010 \\
\hline
\end{tabular}

